\title{
Systematisation: Social Memories and Social Oblivion
}

The second part of this study focuses on locating the concept of oblivion in the context of memory and remembrance in a genuinely sociologically way. Furthermore, there is a focus on oblivion as the problem of a social or general loss of knowledge. Firstly, this section will present an overview of sociological theoretical perspectives of social memory and its equivalents. Then sociological theories will be assessed for explicit and implicit oblivion motifs. As the concept of time plays a role also for sociological theoretical work, we will look for stimulations by and connection points to sociological concepts of time in the course of a second excursus. Then in the final section, a synthesis of these perspectives will be made, resulting in developing sociological search heuristics for phenomena of social oblivion.

\subsection{Structural Aspects}

Thus far, when exploring oblivion, the concepts of memory and remembrance have frequently been discussed. However, a closer look gives the impression that contrasting oblivion and memory, as it is common both in everyday thought and encountered with several scientific concepts of memory, is not helpful. Perhaps it is more obvious to imagine memory and oblivion as the poles of a dialectic relation. Remembrance may then be the synthesis of the opposition of memory and oblivion. Comprehending this seems counterintuitive, insofar as in everyday language, memory is rather associated with the idea of space. Accordingly, memory is usually understood to be the storage place of the impressions of past events. Oblivion, on the other hand, refers to a process (such as in the sense of losing something, being cancelled, disappearing, deleting).

Also, within sociological theories of memory, we find hints at a spatial understanding of memory. In contrast to everyday thought, in which memory is often understood to be a structural or functional concept. Memory constitutes and coordinates remembrance, but if and in how far it is also in charge of oblivion is a good question and requires terminological-theoretical clarification. 
However, from now on, it will no longer be helpful if we try to specify any universal conception which is also connectable to everyday language. After reaching a certain level of differentiation, understanding will become different according to each specific orientation of individual disciplines. Similarly, the terminological specifications of the respective technical languages will be made, which are no longer in line with everyday understanding. Whereas up to here, the analysis has been focusing on generally assessing the material on oblivion. In the following, the relation of the concepts connected by the triad of memory, remembrance, and oblivion shall be analysed. In this context, the arrangement of the three concepts is neither something like integrating remembrance and oblivion under "memory" as an umbrella term nor is it a dialectic which might contrast remembrance as a "thesis" to oblivion as its "antithesis", to then come to memory as a "synthesis". Instead, we will assume a tensed relationship between memory (as the "thesis") and oblivion (as the "antithesis"), as the synthesis of which there happens remembrance.

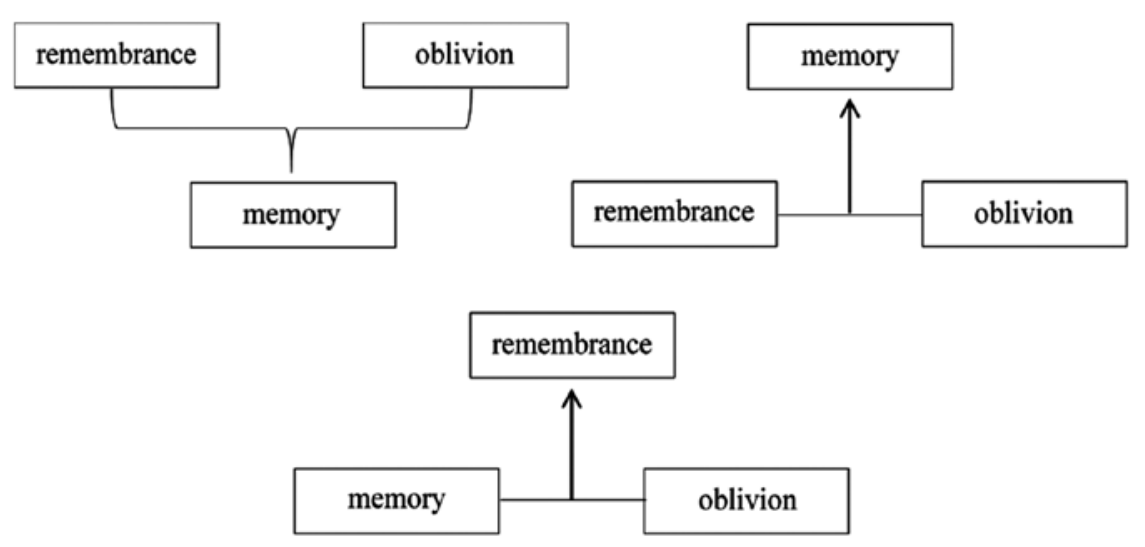

Figure 1 The triad of memory, remembrance and oblivion - memory as a "clamp", synthesis and supplement

In considering different theoretical perspectives, it will become evident that there are individual terms that have more or less fulfilled the function of memory concepts among the toolset of sociological concepts. In comparing these concepts and extracting typical features in each case, it is possible to specify a sociological understanding of memory. Then, such a concept may serve as a foundation for deciding about the "place" of memory and developing an independent sociological concept of oblivion.

Many references to memory are to be found among sociological theories; still, they have only basically been presented since the introduction of collective 
memory by Halbwachs. Some well-known sociological approaches may be called explicit; though, no independent approach connecting to these pioneering works has been worked out yet. Rather, explicit concepts of memory have the status of documenting how sensitive each respective theory is towards issues of time and persistence. In the following, a basic concept of Halbwachs's theory shall be presented: the concept of the social reference frame. Then the memory-theoretical foundation of the social-phenomenological basic concept of the "social stock of knowledge" will be discussed in the context of the theory of social systems.

\subsubsection{Collective Memory}

The concept of collective memory, according to Maurice Halbwachs, rests most of all on the concept of the social reference frame. In the German debate, this is less striking because the original title, "Le cadres sociaux de la mémoire", in which the frame concept is central, was changed into "Das Gedächnis und seine sozialen Bedingungen" [Memory and Its Social Conditions] for the German edition. Halbwachs makes use of the frame metaphor as a basic element of the theory of collective memory. As a result, there are two different yet mutually related perspectives: initially, Halbwachs uses the frame concept, which he found in Bergson and Durkheim, only to label a specific kind of social relations. Elsewhere, the frame concept is then used as a dimension of social orientation by reaching back to the basic categories of time, space, language and experience. ${ }^{1}$

The social reference frames of family, religion or social class are only exemplarily presented by Halbwachs. His considerations are based on the assumption that specific ways of associating are also connected to certain social orders. These social orders do regulate not only behaviour but also structure the individual's remembrance. In other words, as a result of some thought impulse or sensual stimulation, we have no choice but to relate our remembrance to our knowledge of social orders. Thus, the family frame does not only represent all related individuals as well as their relations to each other. It covers all the typical meanings of e. g. the different genealogical relationships.

The framework of family memory is made of notions - notions of persons and of facts - that are singular and historic in this sense but that otherwise have all the characteristics of thoughts common to a whole group and even to several groups. (Halbwachs, 1992, p. 83)

1 A detailed analysis of the memory-specific use of the frame concept was carried out by Oliver Dimbath (2013) angestellt. 
The situation is the same for the frame of religion or religious community and for that of social power structures and power relations as they find expression by the class or strata structure of society. Social order as a habitual, sometimes intensively learned and exercised knowledge is available for the individual as a basic framework for being provided with orientation in current situations. The perception content, which under situative conditions happens by way of sensual impressions, can only be processed in the context of this social knowledge structure. Whatever we experience - we automatically compare it to what we have already been knowing. If this comparison happens consciously and for the purpose of being attributed with meaning, we understand it as remembrance.

The second perspective of social reference frames covers culture-specific ways of articulation. It is about language and interpretation guidelines along the orientation dimensions of time and space, which are fundamental for many cultures, or about the categories of experience. These analyses make Halbwachs conclude that " $[\mathrm{n}] \mathrm{o}$ memory is possible outside frameworks used by people living in society to determine and retrieve their recollections" (Halbwachs, 1992, p. 43).

If the frames of language or space cannot be addressed, no memory is possible. Accordingly, our memories fade away after the house where we have spent our childhood has been demolished. However, not the house itself provides the frame, but this is done by the perception and knowledge of the house as well as by the system of meanings the house of the childhood days may have for any human. The frame of space - only exemplarily mentioned here - is a stable form or knowledge structure, functioning as a constantly renewing projection surface of remembrance.

Thus, collective memory must be understood as a group memory which, being very close to Durkheim's concept of collective consciousness (Durkheim, 1984), represents a group-specific knowledge structure and, thus not least, the coherence principles of these groups. In contrast to Durkheim, for whom collective consciousness is an evolutionary order structure, Halbwachs is interested in remembrance and memory being construed from those social structures whose genesis can be better explained by attributing them to specific group arrangements.

\subsubsection{Context of Experience and Stock of Knowledge}

In the social-phenomenologically oriented sociology of knowledge - in particular, in Alfred Schütz - we find several passages hinting at a sociological theory of memory. Schütz's considerations start with the project of specifying Max Weber's understanding of sociology, which Schütz attempts to achieve 
by including Edmund Husserl's theory of "inherent consciousness of time" and Henri Bergson's concepts of "continuity". 2 This allows for reflecting on the timeliness of action, for which biographic having-become is given as a reason and drafted by pre-memory in the sense of a future action that is imagined by reaching back to one's own experience, as being completed. In the course of his considerations on the "place" of the knowledge available for action plans, Schütz works out the concept of the subjective experiential context, which, in view of the social group, can be aggregated into a social stock of knowledge. Finally, the concept of the lifeworld - also adopted from Husserl - marks the horizon of all imaginable experience and thus the limits of the world.

Similar to the social reference frames, which constitute collective memory in Halbwachs, the acting subject in Schütz reaches back to a stock of existing knowledge that is pre-structured. Thus, knowledge is not necessarily explicable but may become habituated as a routine and may evade reflection by the individual. ${ }^{3}$ The subject's experiential context comes from his/her immediate experiences and the culturally sedimented experience of everyday or general knowledge communicated by others. Even such a kind of knowledge is at first perceived through experiences - still, these experiences may consist of stories and narrations by others.

However, Schütz's considerations become memory-theoretically significant elsewhere. In the context of his attempt to understand action, Schütz also tries to give an answer to the question of why only certain aspects of knowledge are chosen from the experiential context and why these aspects do not only predetermine the action to be planned but also subjective perception as a whole. ${ }^{4}$ Schütz finds an answer with the help of the concept of relevance,

2 On this see Schütz's (1967) fundamental work on The Phenomenology of the Social World as well as the preliminary studies on Life Forms and Meaning Structure (Schütz, 1982), which were published posthumously. These early works were commented on in detail in the newly edited complete edition, edited by Matthias Michailow, Gerd Sebald and Ilia Srubar (2006). A short overview of the theory of memory presented in "Lebensformen" is to be found in Oliver Dimbath (2009).

3 On this see also Michael Heinlein and Oliver Dimbath (2010).

4 A similar memory theory which is certainly oriented at Schütz, although his name is not explicitly mentioned, is worked out by Anthony Giddens (1984, pp. 48-49) who, in line with the phenomenological tradition of theory, distances himself from utilitarian-rationalist action concepts. He structures the action-guiding consciousness into three levels, the first of which is called the "discursive" one because it encompasses all facts which can be expressed by words. He calls the second level the "practical" one, as it addresses those schemes which guide perception. These schemes develop from the body moving through time and space, without any possibility to explicitly name this knowledge. Finally, the third level represents the "unconscious", where - quite in the sense of Freud - an inhibition prevents that something is addressed and emphasized into the consciousness. From this consciousness concept 
which describes a socially constituted selection mechanism. Accordingly, for any culture-specific relevance, structures can be stated which, hierarchically organised, provide the actor with orientation in each current situation. ${ }^{5}$ However - although occasionally Schütz gives the impression - such a way of determining the situation cannot be exclusive of a declamatory-reflective or cognitive nature. By the example of the concept of the because-motifs, which are derived from lived experience and are usually not reflectively accessible, Schütz demonstrates his awareness of the great weight of habitual knowledge (Schütz, 1967). Transferred to the concept of the social stock of knowledge, any identification with the cogitable, linguistic, and thus symbolic knowledge of culture would reduce the range of this idea. The social stock of knowledge including those relevance structures as making it relevant for actions - serves as an orientation system that has developed from individuals' past experience and is modified by each further experience. We may not assume that this stock of knowledge grows or becomes ever further differentiated. Instead, via the connected relevance structures, it is a selection system always present while constantly changing when it comes to practical being-in-the-world. It covers drafted actions as well as pre-reflective action according to habits, routines and practices. ${ }^{6}$

First, this reference to experience has just been piled up. Still, it is further complicated by systems of symbols such as language, whose development happens mostly independently of the individual and becomes evident as being institutionalised in particular because of the changed chronological perspective resulting from scribality. Indeed, the meaning of linguistic signs is inseparably connected to the interpreter who attributes meaning to them; however, the durability of the medium of writing suggests - at least if compared to oral communication - the possibility of preserving some elements of meaning. In other words: through language and, most of all, writing, individual aspects of the relevance structure may be preserved over more extended periods, which

Giddens concludes a memory concept which understands the consciousness to be "sensory awareness", the memory to be the "temporary constitution of consciousness", and memory to be a "means of recapitulating past experiences".

5 Considerations on the problem of relevance became part of the late work The Structures of the Life-World which was completed by Thomas Luckmann (Schütz \& Luckmann, 1973). From Schütz's literary remains, however, also a voluminous manuscript titled Reflections on the Problem of Relevance was published posthumously (Schütz, 1971). The relevance concept has been dealt with from memory-sociological points of view by Mathias Berek (2009), Michael Heinlein and Oliver Dimbath (2010), Marco Schmitt (2009) as well as Gerd Sebald and Jan Weyand (2011), among others.

6 See the considerations by Alfred Schütz and Thomas Luckmann (1973) on habitual knowledge in The Structures of the Life-World. 
is why we must assume that a change of memory-specific selectivity happens at a different speed. Occasionally this results in irritation because sometimes meanings change faster than semantics.

Crucial for the social-constructivist theory of memory as implicitly laid out in Schütz's work is connecting the development and change of collectively binding relevance structures to the subjects, in the sense of a methodical individualism. However, these subjects' ways of experiencing past experiences and their communication about these experiences result in structures of meaning that the individual cannot control. The social stock of knowledge resulting is no gigantic hard disc where everything which has ever been known is archived and kept available. Rather, it is the horizon of the lifeworld which constantly or in each moment newly constitutes itself.7 The stock of knowledge becomes memory only with the help of its immanent relevance structure, which, analogously to the synaptic structure of the brain, performs selections according to certain rules and path-dependencies. These selections become apparent only through observable behaviour or actions, after having constituted perception and the preconditions for those experiences as being transformed into experience. ${ }^{8}$ Thus, it becomes furthermore apparent that memory may be understood as an aspect of the stock of knowledge that deals with the present and determines each currently running selection. In each present, the social stock of knowledge constitutes itself by reaching back to past events or to their sedimentation and takes care that they are changed into experience. ${ }^{9}$

The works of Alfred Schütz provide several reference points for the development of a theory of social memory, remembrance and oblivion. It is currently only about an overview of essential motifs; hence, we will turn towards other theory offers. We will have to come back to Schütz several times anyway.

7 Schütz adopts the concept of the horizon from Husserl and differentiates it further - most of all in the context of memory - by e. g. distinguishing a horizon of the future and a horizon of the past. In this context, lifewordly thought aims first of all at the horizon of the future (Schütz \& Luckmann, 1973, p. 22).

8 In Alfred Schütz (1967, p. 79), experience has got nothing to do with practical or routine knowledge. Rather, it is the meaning-component of the experience. Only after, against the background of previous experiences having been transformed into experience, an experience has been classified, it becomes meaningful and thus experience.

9 Thus, it does not matter if now historical or future issues become a topic of the stock of knowledge. The stock of knowledge consists of nothing else than of things past and is the foundation of any orientation in the present, no matter if this orientation aims at current behaviour, action, or at the future. This contradicts Mathias Berek (2007, p. 71) who understands memory to be the past-related element of the stock of knowledge. 


\subsubsection{Social Systems}

The development of a structural-theoretical sociology of memory goes back to Niklas Luhmann, who rests on the same phenomenological foundations as Schütz when it comes to grounding his theory of social systems: also for Luhmann, whose early works still document his intensive reading of Schütz, Husser's phenomenology is the starting point. For example, he reaches back to the concept of the horizon; however, he takes it out of any cultural or societal context and reduces it to topical communication contexts. Thus, Schütz's stock of knowledge is parcelled into systems of meanings according to the functional needs of communication. Furthermore, such a diminution of the stock of knowledge is in line with a radical reduction of the selection needs represented by the relevance structure: the system recognizes as relevant only that what is relevant for its continued existence (autopoiesis) from its follow-up communication. The system's memory continuously assesses if new environmental impressions are coherent with existing processing structures. In other words: in Luhmann, Schütz's social stock of knowledge becomes a topical communication structure growing out of its history yet continually operating in the presence. At the same time, the system-specific relevance structure is topically reduced to a horizon that is determined by the social function of the system of meaning and whose selectivity can be depicted by way of a binarily coded fundamental distinction. If the basic distinction of the scientific system is determined according to true/untrue, communication is continued only where it is possible to communicate under this premise.

Now Luhmann, who vehemently tries to distance himself from a social phenomenology à la Schütz in his later works, introduces the concept of memory to reshape the selection aspect by reaching back to his time theories as an element of his phenomenological "roots". In the context of the analysis of different communication systems, this concept is used in many recent works to understand the independent history of antipoietic systems. ${ }^{10}$ However, using the concept of memory as an auxiliary construction is inconsistent, after all - mainly if the phenomenological classification system is wholly left and the memory of the social system is defined as discriminating against remembrance and oblivion. By the social memory determining what a system must remember and what it must forget, after all, it is reduced to the function of the

10 One work where Niklas Luhmann deals explicitly with these issue is his essay "Zeit und Gedächtnis" [Time and memory] (Luhmann, 1996). Since the end of the 1980s, however, a number of publications present short considerations on the memory of the social system. 
system's self-historicisation, without the crucial yet reductionist memory function of binary coding still being in focus. ${ }^{11}$

Also, the considerations by Niklas Luhmann provide a number of starting points for a theory of social memory, remembrance and oblivion. However, as not even Luhmann developed any explicitly sociological theory of memory and some aspects seem unconnected, these lines are not meant as a comprehensive appreciation. However, where it is appropriate, we will reach back to the many memory-sociological inspirations from the field of the theory of social systems.

\subsubsection{Sociology Without "Memory"?}

Given the Durkheim-based works by Maurice Halbwachs on collective memory, it would be inappropriate to assume that sociology mostly lacks a concept of memory. Not only Halbwachs but also Schütz or Luhmann are provided - as demonstrated - with at least basically worked out concepts of memory or remembrance. ${ }^{12}$ Although it is likely to result in misinterpretations if any structural concept from the field of sociological theories is associated with memory, we cannot help but add a memory function as organising the reference to things past to any structure or any explanation of structuring or structural formation. Many theoretical drafts of structure implicitly mention memory equivalents, or the latter appear under different names - however, a terminological integration into the concept of memory, which is then, dependent on context, in need of differentiation again, might help with creating a more distinct and most of all universally applicable terminology and with getting rid of the hardly helpful multilingualism in this field. In other words: we might ask how the reference to the past is conceptualised in processes aiming at structure formation - may it be structuring, institutionalisation, selforganisation (autopoiesis) framing, routinisation, classification, culturation, habituation, objectivisation, reification etc. Furthermore, the motif of memory is found with sociological diagnoses of processes and times, which brings us to the question of the premises the construction of certain pasts as connection points for stating new developments of theories of social change is subject. Thus, the focus is on modernisation, rationalisation, differentiation,

11 On the many ways of making use of the memory concept in Luhmann see Oliver Dimbath (2011a).

12 For an overview see Marco Schmitt (2009) or Michael Heinlein and Oliver Dimbath (2010). For the time being, probably the oldest draft of a sociological theory of memory, which belongs to the pragmatist tradition, by US American social activist and social researcher Jane Addams of the year 1916 has been met with little response (see Addams, 2002). 
pluralisation, disciplining or civilising, each of which has its own specific ideas of social past and future.

What is remarkable with the memory equivalents developed in sociological theory is that they are rather structural concepts than storing metaphors. In other words: these theories hardly refer to "places" where past events are stored or archived. As the structure of the social order continuously updates itself that is, it adjusts to its respective present - reaching back to manifestations of the past seems to be hardly relevant. Then, a sociology that presents itself as being presentist may be satisfied with describing or reconstructing principles of social order, ${ }^{13}$ whereas speculations about their development may be left to historically interested sociologists or historians. ${ }^{14}$ It is thus a natural question why sociology needs a memory concept at all. In terms of social theory, the structure of social order would have to be understood as the storing place of evolutionarily "grown" regular knowledge, which is imprinted into the structure of social institutions - a metaphorical reference to a place.

Another reading understands memory as a function and implies it with any structure. This is meant to say that any social structure provides certain selection mechanisms for the production of each current memory. Aspects of social structures address subjective experiential contexts with actors and, this way, attract attention. At the same time, once having been perceived, they provide classified categorised orientations for action, the meaning of which results from the history of each structural aspect. Thus, we may say that culturespecific order structures seem obvious for standard situations for a start.

However, individuals do not act blindly according to allegedly clear guidelines, but they interpret them by themselves and according to their further proceeding. Consequently, social memory provides them with specific selection possibilities both to define a situation and to attribute meaning in view of further action. In this context, violating the order is a possibility that may be considered and may result from subjective experience being confronted with sometimes internalised or objectivist social patterns of behaviour. In such a case, however, the memory function does not work in the sense of referring to facts. In whatever way they may be stored and fixated, it rather results from a combination of two knowledge-related selection mechanisms: a biographic one and a situation-specific one at the level of constituting meaning. It is beyond dispute that both kinds of selectivity cannot be understood other than in the social sense - that is, as the results of socialisation and learning processes. However, it is doubtful if this kind of memory should be called functional memory, as it is common in the cultural studies debate on memory.

\footnotetext{
13 On this see e.g. Jan Assmann (2002).

14 See Rainer Schützeichel's (2004) characterisation of the task field of historical sociology.
} 
According to Aleida Assmann, functional memory consists of the present stock of things past that is constantly in use and must thus not be forgotten - a kind of memory necessary for the functioning of cultural life. ${ }^{15}$ Concerning a sociological concept of memory, however, memory functions by certain contexts of meaning which have been construed or constituted in the past, selectively orienting the contingent interpretation of current situations - in view of further action and further communication. Thus, the function of memory may instead result from imagining a selection performance in the course of which it is decided what is supposed to be relevant in a given situation. It is thus not about knowledge being "stored" at a particular place.

\subsubsection{Memory Equivalents}

Based on the distinction made so far, a look at some basic concepts of the conceptual toolset of sociology makes it evident that each of these concepts may be considered a memory equivalent. It is particularly revealing to ask about the functional aspects of each of these specific selection mechanisms. On the one hand, such an analysis will reveal systematic-conceptual differences between concepts which, at least partly, refer to the same. On the other hand, we may identify further aspects of different memory functions. In the following, only exemplarily and to demonstrate the equivalence assumption, the concepts of frame and interaction structure (1), identity and narration (2), taboo, ritual and myth (3), practice, field and habitus (4), network (5), discourse and dispositive (6), and institution shall be made subject to a necessary cursory assessment. This list could be extended by other concepts, such as social role, norm, value, custom, and more. However, here we will be satisfied with assessing the former concepts, as already by them, the crucial motifs of the reference to the past can be demonstrated.

The, at first sight, at least terminological similarity of Goffman's frame concept and Halbwachs's concept of the social reference frame has frequently been stated, particularly by the cultural studies looking for being socialtheoretically grounded. ${ }^{16}$ Probably the first and, given its shortness, topically

15 Aleida Assmann connects the canon to functional memory. "For canonisation means additionally the trans-historical self-obligation to repeated reading and interpretation. Accordingly, despite accelerated innovation, the stocks of functional memory are still part of the curricula of educational institutions, of the playlists of theatres, are presented at museum halls, are part of concerts at concert halls and of the programmes of publishers. What has its place in the functional memory of a society may claim to be again and again newly presented, exhibited, read, interpreted." (A. Assmann, 20o6, p. 56)

16 Hints which are not meant as a comparison of theories but which are first of all about terminological convergence are to be found e. g. in Dietmar J. Wetzel (2009), Harald Welzer (2008) who, however, interprets Goffman as a memory-relevant theoretician in a different way, and Jan Assmann (2011). 
most distinctive analysis is to be found in Jan Assmann, who points out to the fact that "Halbwachs runs along surprisingly similar lines to E. Goffman's theory of 'frame analysis'" (J. Assmann, 2011, p. 22).

Without any further comparison of the framework concepts of Goffman and Halbwachs, ${ }^{17}$ light shall be shed on some crucial aspects of the frame in Goffman in the following. First, we will discuss to what extent it is a memory equivalent - despite any references to Halbwachs. Goffman, who adopts his frame concept from Gregory Batson (1972), presents the following definition:

I assume that definitions of a situation are built up in accordance with principles of organization which govern events - at least social ones - and our subjective involvement in them; frame is the word I use to refer to such of these basic elements I am able to identify. (Goffman, 1986, pp.10-11)

However, this definition does not result - as might be suggested by the Thomas theorem, which Goffman criticizes $^{18}$ - in any subjective-decisionist routine of defining a situation. Instead, the result is supra-individual principles of order - and here, this is expressed by "we" - with the help of which the individual can orient him or herself in a given situation. Consequently, the frame answers the question "What is going on here?" and the question about action patterns, that is, "What am I supposed to do?". That Goffman is less an interactionist but rather a Durkheimian in this concern has been stated several times (Lenz, 1991): frames are - although being sociologically construed - sociological facts which in certain situations are perceived as being given and in case of ignoring them result in sanctions. The behaviour is inappropriate or does not fit the frame. On the one hand, the frame as a structural concept would be a supra-individual stock of knowledge being only partly open to reflection and suggesting situation-appropriate interpretations and ways of behaviour and organising everyday experience. On the other hand, based on considering experience the schematisation or classification of experiences, both a concept of social time and memory has been presented by the frame concept. If we want to understand the interaction order represented by frames ${ }^{19}$ in historical terms, we would first have to reconstruct the frames and pursue their references to other frames until no more reference to any modulation could

\footnotetext{
17 On this see Oliver Dimbath (2013).

18 The Thomas theorem (Thomas \& Thomas, 1973) says that concerning its consequences a situation is real if it is considered to be real.

19 In a speech summing up his scientific work, Goffman gives a hint that his research interest was first of all in understanding the interaction order - against this background, the frame concept must be understood as a structural-theoretical element (see Goffman, 1983).
} 
be found. According to Goffman, then we would have arrived at the primary frames. ${ }^{20}$ Such a reconstruction is at the same time a presentist construction of a past in the sense of frame history and an analysis of the social memory, in so far as experience-constitutive, classified interpretations or combinations of interpretations are investigated.

We may thus state that - according to the micro-sociological analysis of interaction structures unfolded in Goffman - the frame concept must be understood as a structural concept providing the individuals, which are entangled in interactions with adequate rules of conduct. These rules are valid for interaction types, are "habituated", but the actor may reshape them at any time. That is, frames provide a memory of social behaviour in the context of interaction relationships by providing culture-specific predictabilities. These predictabilities can sometimes be sanctioned, but they may as well be disappointed or evaded. In so far, Goffman's framework concept is a micro-variant of Halbwachs's framework concept as unfolded according to Durkheim's and Bergson's earlier use of the concept.

The identity concept of sociology refers less to consistency and consubstantiality or synchronicity in the sense of simultaneity and diachronicity as they are discussed in philosophy (Straub, 2001). Rather, here the knowledge of the individual, which most of all refers to him/herself and his/her own history, is at the fore. ${ }^{21}$ From a sociological or social-psychological perspective, this is connected to playing social roles or - in Simmel's word - participating in social circles. ${ }^{22}$ Identity as a socially and socialisation-communicated concept of oneself becomes manifest by autobiographical narrations. However, when looking beyond the individual, we may find similar narrations also among groups. Also, collectives create narrations referring to themselves and their histories - then we speak of collective identity. For both identity references, there holds that, in the sense of synchronous identity, the coherence of "moral and aesthetic systems of maxims" and, as diachronic identity, of continuities is usually created through narration (Straub, 2001, p. 270). ${ }^{23}$ In any case, the narration appears as the practice of constituting social time. Thus, identity means

20 See Erving Goffman (1986) who assumes primary frames as the basic patterns of social interaction which are permanently modified in the course of the everyday organization of social action - such situatively adjusted or derived frames are what he calls modulations. Accordingly, one may suffer from a loss of identity - caused by a damage of the brain or in the form of retrograde amnesia. Then one does no longer know who one is.

22 As it is well-known, Simmel (2009) conceives identity as the intersection of social circles.

23 Here we must particularly consider the idea of the collective unconscious according to C. G. Jung (1975). 
the construction of the personal and collective present by selectively reaching back to experience coming from past and "processed" experiences and events.

Self-referential knowledge is versatile due to its implicit presentism; nevertheless, it is perceived as being relatively static. The reason for this is the implicit, path-dependency-based, continuation programme - one stays "true" to oneself. This is why any memory-interested analysis must deal with processes of identity formation and changing identity and must ask which selection mechanisms are at work when a consciousness (narratively) makes sure of its own identity. Notwithstanding any narration-communicated attempts at self-consistency, we may hardly speak of any consistent and coherent selfnarration. Rather, the latter seems to depend both on the perceived situation and the social living conditions. ${ }^{24}$

Concepts of collective identity are closely connected to the concept of collective memory. Collective identity refers to the group identity of social groups as it is articulated through narrations. Among others, this is based on the assumption that collective identity comes from collective memory. Accordingly, Lutz Niethammer understands the concept not only in the sense of Halbwachs and the idea of collective consciousness in Durkheim but also in view of identity being an artificial product of political agitation in view of the idea of the unconscious in Freud's psychology (Niethammer, 2000). However, collective identity is not the only memory-relevant concept for the description of group-related identities. Also, there is research on group cohesion or national characters as well as on mentality point in this direction. ${ }^{25}$ It seems to be little help to discuss a static image, a "snapshot", instead of asking about those constitution or selection mechanisms to be found with any collective culture as creating collective self-descriptions.

Sociological considerations on knowledge structures such as taboos, rituals and myths start from the research by Durkheim (2008) and are continued most of all by his disciples Henri Hubert and Marcel Mauss. From the perspective of sociology, making use of these concepts seems to belong to the "scope" of anthropology and ethnology. However, by sociology adopting the works by Edward E. Evans-Pritchard, Claude Lévi-Strauss or Mary Douglas, who refer to Durkheim, these concepts are being "reimported".

24 The basic idea is an old one: existence determines consciousness. Yet still, it is the foundation of post-modern identity concepts as being negotiated in the context of patchwork identity (Keupp, 1989) or the quasi subject (Beck et al., 2003).

25 See e. g. the contributions in the volume on Transformations of Shared Identity edited by Reinhard Blomert, Helmut Kuzmics and Annette Treibel (1993). 
Durkheim extensively discusses the concept of taboo. ${ }^{26}$ By a taboo, he understands - like later also the anthropologist and ethnologist Mary Douglas specific ritual bans which secure an existing order by banning certain actions. At first sight, the taboo, due to which entering certain places as well as touching, looking, addressing or just speaking the names of holy persons and sacred objects is banned, looks like a predecessor of the concept of silencing. This impression becomes stronger when in everyday language, the taboo is understood to be a ban on making something the subject of discussion, meant to prevent the memory of events associated with shame and disgrace - "it is not mentioned anymore".

In the context of religious rituals, however, the function of the taboo looks different: taboos separate the sacred from the profane or purity from impurity. Accordingly, in the religious context - which constitutes the group's cohesion, after all - nothing profane is allowed, and in the context of everyday life, nothing sacred is allowed. In everyday life, precisely the strict boundary between the sacred and the profane constantly reminds to - and indeed also because it makes life complicated - the religious, so that the taboo has a memory function that is oriented at the group's collective identity. At the same time, the taboo has an oblivion function when, due to its high sanctioning power or to realised self-constraint, it has such a highly selecting effect that even apparent action alternatives are always suppressed and thus left to oblivion. ${ }^{27}$

Mary Douglas calls the ritual an "action and beliefs in the symbolic order" (Douglas, 1996, p. 2), in the context of which it stays out of consideration if those participating in the ritual feel obliged to do ${ }^{2} .{ }^{28}$ For a sociological understanding of memory, the concept of the ritual is fruitful in two ways: at first, the ritual with its sequence of traditional, routinized symbolic actions, which are supposed to make a group's coherence last, functions as a material, social

26 Although in the first, French, edition of his work of the year 1912 Durkheim (2008) made an outstanding contribution to sociologising the concept of the taboo, it must be pointed out to the fact that in 1912 and 1913 Sigmund Freud published his works on Totem and Taboo. Although the two authors come to different conclusions, the basic assumptions and starting points - in particular the working out of the concept of taboo - are very similar to each other.

27 Here the paradigm change from a religion-oriented interpretation of social situations to a language-oriented interpretation (linguistic turn) appears as an oblivion function, as the concept of silencing is clearly less differentiated than than concept of the taboo. The taboo includes also non-linguistic actions. Only the practical turn catches up with it.

28 In her considerations on the concept of ritual Mary Douglas (1996) is first of all interested in justifying the social phenomenon which, in her opinion, is under threat. For this purpose, she delimits it from everydy and most of all also from sociological readings of her time. Today her works are mostly met with agreement, also in sociology. 
knowledge structure in the long run. Remarkably, however, this is not about meaning as explicit or explicable knowledge but, on the contrary, about highly symbolic actions which may nevertheless not be challenged. The function of these actions is the continuation of collective identities, even if the original meaning of this socialisation has long been forgotten. Thus, there is the second aspect: rituals are "stored", collectively schematic knowledge structures with a specific oblivion function. They can do without perpetuating meaning just by continuing the social practice.

Also, the roots of the concept of myth go back to the research of so-called "primitive" cultures, which started in the 19th century. However, as the myth is tied to linguistic expression, it is somewhat close to the concept of narration. Being a legitimating narration about institutionalised contexts, for a long time, it referred to magical or religious contexts and has returned to the sociological debate only by way of new contexts of meaning, such as that of everyday myths (Barthes, 1972), or the mythologised formal structures of organisations. ${ }^{29}$ When representing the institutional structure of socialised social structures, myths appear as narrative or symbolic ways of expression and thus as linguistic manifestations of the selectivity of social memories. At the same time, they also serve as oblivion generators ${ }^{30}$ that, due to their legitimation function, systematically suppress alternative interpretations and are thus capable of taking care that "violations of the norm" are soon forgotten.

We may understand the concepts of ritual and myth as being closely connected, oblivion - or better: making forget - being a crucial feature of both. By reaching back to René Girard's meconnaissance theorem (Girard, 1986), it is possible to demonstrate that the forgetting of perhaps traumatising or otherwise annoying or subversive narrations as well as their overwriting by relieving, stabilising narrations, which is purposefully inherent in the myth, also holds for the concept of ritual. Meconnaissance may be translated as making forget, concealing or ignoring. For example, greeting rituals make those participating in this interaction forget their mutual aversions of hostilities, at least for a short time or temporarily. As it is sometimes stated in the public debate and is also diagnosed by Douglas, the criticism of rituals aims at their stabilising while at the same time affirmative effect in the sense of covering up the "actual" situation. Nevertheless, it may be stated that a "certain degree of meconnaissance is part of society" (Korte, 2011, p. 156) and is necessary to

29 See John Meyer \& Brian Rowan (1977), although concerning these authors, in the context of the theory debate of German sociology we would rather speak of ideology instead of myths.

30 Martin Endreß (2011) coins this term in the oblivion-sociological context. 
maintain the social order. The other side of meconnaissance is that rituals celebrate a certain memory over more extended periods, a commemoration. According to Durkheim, religious festivals are - in a ritual way - reminders of the coherence of the community as it has been communicated by myth. However, in everyday life, it is sometimes more practicable, or not even necessary, to temporarily forget the remembered values - that is, only until the next festival.

The question of how far the theory of practice - as supported by Pierre Bourdieu, particularly the concepts of the social field and habitus - may be understood as social memory has already been discussed by several authors. ${ }^{31}$ We may state that both the concept of practice and the habitus concept address the aspect of an incorporated, i. e. imprinted into the bodies of the individuals, social structure. Without applying the tool set of social-scientific interpretation, this is hardly accessible for reflection by the individual or the group. Nevertheless, the here discussed routines and behaviour patterns are socially recognized and understood routinely or mostly without reflection. That habitus is structured and structuring structure (Bourdieu, 1986a), makes it at first look like a structural concept in the sense of a knowledge store, its structuring and perpetuating effect mainly staying in the dark.

However, even practices and habitus are subject to permanent change, which raises questions about the selection processes being at work there. In contrast to many other theory offers, by Bourdieu, by the concept of the social field, we find a systematic tool for precisely analysing those selection processes as constantly shaping practice and habitus. Furthermore, the possibility of the actor in the field calling these structures into question and perhaps changing them is taken into consideration. ${ }^{32}$ Bourdieu states, that habitus

$[\ldots]$ is the presence of the past in the present which makes possible the presence in the present of the forth-coming. It follows from this first that, having within itself its own logic (lex) and its own dynamic (vis), it is not mechanically subjected to an external causality, and that it gives a freedom with respect to direct and immediate determination by the present circumstances (in contrast to what is asserted by mechanistic instantaneism). (Bourdieu, 2000, p. 210)

31 Extensive considerations which might reconstruct crucial aspects of the memory concept which Bourdieu himself does not explicate have been made e. g. by Alois Hahn (2010), Jörg Michael Kastl (2004) or Marco Schmitt (2009). Remarkably, Paul Connerton (1989) unfolds a theory of body memory which mentions Bourdieu only in passing and also makes use of a habitus concept and a concept of practice.

32 In the course of an extensive study, Herbert Willems (1997) works out the obvious parallels between Bourdieu's habitus concept and Goffman's frame concept. 
The two concepts of field and habitus describe an interaction in which habitus develops from incorporating specific features of the field while being reproduced and modified repeatedly. Thus, the resulting and perpetuating structure is not inflexible, not at least because of this, the analysis of the constituting processes is revealing. How and via which selection mechanisms are social powers inscribed into the bodily practices of individuals?

The incorporation of practices, the development of habitus, is determined by the requirements of the social field and happens by way of continuous repeating or practising. In other words: one incorporates or "automatises" ways of behaviour that are promising within a social context. Because one can behave this way "in his/her sleep", after all, only the body is the storage place - and not reflecting intellect. At the same time, these ways of behaviour are tied to the field and are practised by the individual and also by many. The persistence of habitus becomes obvious, e. g. when a school class that was lead by an authoritarian or charismatic teacher over a longer period meets again after many years. Although the former students, being adults now, are at eye level with the teacher, they may reactivate old ways of behaviour towards their teacher - when the teacher starts speaking or raises his/her voice, everybody is silent.

Apart from incorporating habitus, there is another aspect of memory in the social field: social capital. In Bourdieu, each field is characterised as the stage for a fight for recognition thorugh capital in the broadest sense (Bourdieu, 1986b). That is, in each social field, certain "currencies" are valid, which find expression not only through money but may also consist of relationships (social capital) or competencies and symbols (cultural capital). In the social field, a specific selectivity towards "being valuable", which the actors believe to be objectively given, develops evolutionary. This can be illustrated notably by the institutionalised and objectivist kinds of cultural capital: in specific fields, holding an educational grade or owning a collection of paintings is a symbolic expression of power and influence. This is based on a specific selectivity which is also in line with the relevance structures of this field, while this kind of selectivity may also determine any definition of a situation. In a specific way, the field's past is present in each new situation. In contrast to habitus, the past, which is maintained in the field as power-bestowing knowledge or knowledge for the sake of domination, is tailor-made only in the particular case of incorporated cultural knowledge. Even if they are mutually dependent, the two aspects of memory appear mostly independent. For example, in the case of an actor having been absent for some time, the field-specific knowledge may have changed so that the 
"old" habitus is no longer appropriate to the requirements of the field and may be perceived as old-fashioned..$^{33}$

Since the invention of writing, printing and new communication media and means of transport, the constitution of social relations has ever less been dependant on the interaction among those present. Perhaps this is in combination with corresponding diagnoses of times such as those stating the information, knowledge or network society - one of the reasons why the basic sociological concept of the group has been gradually replaced by that of the network. ${ }^{34}$ However, the fact that the network is less tied to topographic spaces makes a considerable difference. Whereas the interaction relations between physically present group members can still be analysed as dynamic processes among members, the "virtual" network may right from the beginning be understood as a communication system where information runs through nodes or nexuses. If the structure of interpersonal relationships is still very complex in a group, the communication-centred structure of the network only seems to be complex but is located at a small number of different relationship levels. Here, the network concept is derived from the metaphor of the network as it is used for neuronal structures; it is about associations, connectivities and nexuses. Accordingly, the selectivity of the network depends on the extent to which its nodes and connections are activated, whose weight determines both the strength and the direction of the flow between two nodes. These weights may be understood as selection mechanisms as they have either a positive-enhancing (excitatory) or a negative-inhibiting (inhibitory) effect. Furthermore, network models are provided with a theory of associative learning, which aligns with our understanding of schemes or scripts. This theory says that the connecting weights may change according to certain learning rules. Given the network structure, memory is not understood as a core but as a representation of knowledge within the each specific and changeable pattern of its connecting weights (Goschke, 2001). ${ }^{35}$

Whereas the theory of social systems leans strongly towards the network models of the neuro-sciences and integrates the thus developed learning

33 In Marco Schmitt (2009, pp. 68-69) we find a description of this phenomenon which Bourdieu calls the hysteresis effect - practicing the habitus is connected to a certain degree of sluggishness; in fields being subject to much change sometimes the actors with their adjustments cannot keep pace. However, also the above-mentioned example of the class reunion gives expression to a similar pattern.

34 On this see the considerations by Jan A. Fuhse (2006).

35 Semantic networks are not discussed - they consist of semantic relations of symbolically represented concepts. 
theories as memory functions, we find other implicit concepts of memory in other sociological adaptations of the network concept. For example, Marco Schmitt points out the relationist tradition of sociology based on Simmel's "formal sociology" and figurational sociology, according to Elias, in his networkfocused study on social memories. He believes the concept's great popularity is due to Manuel Castells's thesis of the network society. "Now networks are the most flexible, most robust while most extendable kind of social organisation, as technological innovations, neutralise the thus connected disadvantages of the increased need for coordination and the difficulty of centralising resources" (Schmitt, 2009, p. 191). ${ }^{36}$

The core of Schmitt's analysis is the actor-network theory, according to Bruno Latour, as well as the phenomenological network theory, according to Harrison C. White. For both approaches, he works out each implicit memory function. Crucial for the position of actor-network theory is the insight that relationships of influencing - such as actions - may be complex and that not only living actors but also material objects - actants - must be considered. Thus, Latour rejects the insight, supported by Durkheim, that the social can only be explained by way of the social. ${ }^{37}$ In terms of memory theory, such a figure allows for imagining the influence of material objectification - buildings, computers, libraries etc. - as the reference unit for communication or social acting. A social-theoretical grounding of the idea of the network is found by Harrison C. White's (1992) theory. Without being able to discuss the differentiation-theoretical development of the constitution of social networks in detail, crucial references to the concept of the social memory shall be pointed out by reaching back to Marco Schmitt's considerations. Fundamental for the structure of networks is the development of identities, derived from needs of social control and then perpetuated, of those entities as participating in the network. Here, the somewhat wider concept of entity seems to be appropriate, as networks may consist of actors and positions and groups or "blocks" - that is, entities that are themselves networked. ${ }^{38}$ Identity develops within any network arrangement and is further developed also there. In the course of networked communication, there happen performances that consist

\footnotetext{
36 Marco Schmitt is referring to Manuel Castells's (2000) Rise of the Network Society.

37 Bruno Latour (1993, pp. 51-52) tries to further establish the objects, which in Durkheim are only reference points of the social, by introducing the concept of the quasi-object.

38 We are going to make further use of the concept of entity when it is about referring to social phenomena which are individual and collective at the same time and may be routine-guided. Concepts such as individual, group, consciousness or consciousness system, system of meanings, subject, actor or acting person are all subject to restrictions by way of which an active social entirety cannot sufficiently be described.
} 
of an exchange of information and continue and, if necessary, change the identities of the participating authorities. Two crucial processes in this context are integration on the one hand and distinction on the other. This way, the network defines who belongs (or not). Here Schmitt identifies a parallel to Luhmann's memory theory: due to the flexible identity concept of the network theory and by constantly comparing situative influences and the interaction history of the network relations, a mechanism of opening and closing is implied. Thus, the memory of the network develops from the running communication within the network and, by way of a constantly changeable entity and identity arrangement, decides what can or cannot be made congruent with the network's past. It is thus a memory-related mechanism of integration or exclusion (Schmitt, 2009).

We have already pointed out the fact that power-communicated knowledge orders are a structural equivalent to memory. When interpreting everyday situations, the arrangement of objects and classifications appears to be the given order which need not be called into question any further. Michel Foucault's achievement is having exposed the problems of this allegedly irrevocable and eternal linguistic order by asking in an "archaeological" and "genealogical" way. Discourses organise what we are allowed to speak about as well as how we are allowed to discuss. By inscribing not an evolutionary aspect into the development of discourses but also a power mechanism, this order is recognized as being "made" and thus as changeable. By his archaeology of knowledge, Foucault is interested in the historical changes of the meaning of phenomena of social order (Foucault, 1972). His genealogy asks about the conditions and circumstances of these changes. In other words: Foucault moves away from taking static "snapshots" of past orders and discusses the question about the selection mechanisms the constitution of these orders is based upon and which - beyond any historical interest - may be understood to be social memory.

By extending the scope from the discourse to the dispositive, ${ }^{39}$ which represents not only its manifestations by "administrative, infrastructural mechanisms and measures" (Keller, 2008, p. 92), an empirical analysis of the conditions under which the present constitutes itself is provided with another connecting point. With the dispositive's help, other material elements of the discourse can be grasped, can the social memory be made visible in all objects. Reiner Keller's example of the control dispositive of the police, with its traffic lights, traffic signs, police officers or speed cameras, may be understood to be

39 On the concept of the dispositive as well as on the dispositive analysis see also Andrea D. Bührmann and Werner Schneider (2008). 
an empirical approach at the selection mechanism of the social memory. Thus, for a power-communicated definition of a situation, only selected objects are of relevance - others are not taken into consideration (Keller, 2008).

If we consider Emile Durkheim's sociological fact (faire social) the "rightful" predecessor of the concept of the social institution, connecting it to a primarily static model of memory seems to be obvious. Institutions are understood to be relatively stable structural phenomena whose slow change evades the experience of the individual. Nevertheless, they have grown "evolutionarily" and may thus be attributed to the habitual memory of society. It is disputed to which extent they can also be explicated, as there are institutions such as law, which can be clearly named, and others that are rather "unwritten" yet directive, socially effective and sometimes capable of sanctioning.

The sociological development of the concept of institution is characterised by a stepwise distancing from all too inflexible concepts. ${ }^{40}$ Arnold Gehlen believes institutions relieve man from the burden of being a deficient being with weak instincts; by way of repeatable, socially sanctioned rules of behaviour, man is saved from having to decide about everyday necessities to act (Gehlen, 1957). Institutions have a binding effect and are part of the matter-ofcourse and mostly undisputed leeway to act of everyday reason. By the concept of institution, the effect of social memories becomes imaginable, whose selectivity consists of objectivist criteria for action.

\subsubsection{Memory and Social Change}

Not only conceptual-analytical tools of sociology refer to the problem of social memories. Also, sociological figures of diagnosis resulting from the analysis of social change can hardly do without at least implicitly referring to memory issues. Both in the context of sociological diagnoses of processes and in the context of diagnoses of times and the present, it is constantly about time as well as about the issue of the social past. As usually a process of social change which is construed as being typical is in the focus of interest, sometimes differentiated analyses of starting and endpoints retreat to the back, so that after all simple dichotomies such as old and new or traditional and modern are applied. How specific features of both the new and the old are worked out and in which hypotheses about processes of change are developed and discussed can be understood against the background of a social memory function. As already illustrated several times, each thesis can be traced back to a selection

40 In this context, Parsons's concept of institutions must be mentioned. For Parsons, the institution is one of the different "classes and types of governing action [...] because of its special functional significance to social systems" (Parsons, 1994, p. 153). 
mechanism. Moreover, this is true in two respects, as a typical process is investigated and a specific past is construed or invented on which the analysis of the new rests. ${ }^{41}$

That usually such a reflection is lacking may be due to sociology understanding itself as "the" science of modern society as such. Then modernity is contrasted to tradition; social change is understood to be a new beginning. In this context, it is sometimes not considered that the new develops from the old, along path-dependencies. To illustrate this, in the following two older and recently discussed motifs or "driving forces" of social change shall be considered and interpreted in terms of memory theory: generation (1), modernisation (2), acceleration (3), and globalisation (4).

The sociological concept of generation comes from the sociology of knowledge or thought of the school of Karl Mannheim (1952). Connecting to Marx's concept of class consciousness and reaching back to older concepts of generation, he discovers that social change starts out from definable social groups under specific socio-historical conditions. At a particularly sensitive age, the members of generations - here Mannheim assumes an interval of youth or young adulthood - do not only experience similar political socialisation; from the same socio-historical point of view, they similarly perceive social events. Under their respective conditions of growing up, generations develop their own life goals - which, however, are not necessarily shared by the whole age cohort but unfold among individual milieus, groups or generational units. From this, we may conclude that certain groups of about the same age, looking back to similar experiences and thus memories, also develop similar memorycommunicated selectivities for their worldview and choice of action. ${ }^{42}$ The life goals of these different groups may compete with each other - however, their starting point is the same. If one group succeeds with achieving its goal and making it authoritative for the entire generation, the competing drafts may fall into oblivion. Then one generational unit determines the thinking and selectivity of social remembrance until it is replaced by another, younger group and its ideas. ${ }^{43}$

41 This is in line with the insight by Eric J. Hobsbawm and Terence Ranger (1992), according to which traditions have partly be "invented" in retrospect. Here, however, a tradition is invented for the purpose of classification, to be able to distinguish new developments from a yesterday.

Very much to the point, Martin Endreß translates Mannheim's term "unconscious vital inventory" with "latent horizon of implicitness" (Endreß, 2011, p. 67), which goes back to a "manifest common remembrance training".

43 Accordingly, Ron Eyerman identifies with the concept of generation an interaction of social oblivion and remembrance: "Here collective forgetting is as important as collective 
That experience goes with age is in many ways an advantage. That, on the other hand, youth lacks experience means a lightning of the ballast for the young; it facilitates their living on in a changing world. One is old primarily in so far as he comes to live within a specific, individually acquired, framework of usable past experience, so that every new experience has its form and its place largely marked out for it in advance. In youth, on the other hand, where life is new, formative forces are just coming into being, and basic attitudes in the process of development can take advantage of the moulding power of new situations. Thus, a human race living on for ever would have to learn to forget to compensate for the lack of new generations. (Mannheim, 1952, p. 296)

For Mannheim, generation is not just a switch by way of which a specific selectivity, resulting from age cohorts becoming distinct, of maintaining as well as of losing experience and knowledge can be described. Also, the concept of generation provides a systematising understanding of social change resulting from being confronted with the traditional, in the sense of a memory-specific change of society resulting from the coming and going of age-homogeneous groups. $^{44}$

More recent modernisation research usually structures the transition from a traditional to modern society, described by the process concept of modernisation, into sub-processes. ${ }^{45}$ When, just exemplarily, considering the common

remembering for a society's self-reflection; it is in fact the role of the youth or the new generation: to provide society with a fresh look at itself" (Exermann, 2004, p. 71).

44 Aleida Assmann (2002, pp. 185-186) adopts Mannheim's formative power connected to age and even identifies Mannheim as the "ounding father of the research of social memory", without discussing his theory of social change. For her, age cohorts develop group memories depending on location, which then indeed allows for a better understanding of certain historical processes. However, in this context it is overlooked that generational units draw different conclusions at the same time from their past, that they start enforcing them against the preceding generation and, in the course of competing with their fellows, unfold the dynamics necessary for social change.

45 Here we are dealing exclusively with a sociological interpretation of modernisation as a process of a transformation of society - and this just exemplarily. That sometimes the modern age as a cultural epoch is called an age of oblivion cannot be taken into consideration here. Accordingly, David Gross (2000) states that the pre-modern age was dominated by remembrance and that oblivion was negatively connoted. In the modern age, he says, this was reversed, and memory-related values lost their relevance. The function of remembrance, which in earlier times was highly appreciated, lost significance - the reminder him/herself was pathologised as suffering from hypermnesia or as a person without flexibility and being entangled in routine. The authorities Gross refers to, by whose works, he says, this development can be traced back differentiatedly, are Sigmund Freud, Henri Bergson, Walter Benjamin and Marcel Proust. Barbara A. Misztal (2003) describes a similar development towards a culture of oblivion in modern society, beginning with the invention of the encyclopaedia and the nation but coming to an end after 
distinction of rationalisation processes, differentiation, domestication and individualisation (van der Loo \& van Reijen, 1992), it becomes evident that each of them shows memory-specific aspects.

Suppose we understand rationalisation as action in all fields of society being guided by reason or usefulness. In that case, it seems as if there is a selection mechanism of its own kind: rationalisation is characterised by a given state or action context being supposed to require improvement. The use of reason refers to a memory-communicated stating of the deficient, from which there concludes a need for optimisation. Once the innovation has been completed, the old state may be forgotten. What is remembered is only the way of improving, which may be transferred to other fields. Whether there is also a shortcoming might be irrelevant for applying the method. In other words: the trigger for rationalisation processes is determined by identifying a shortcoming, from which there concludes the method of remedying the shortcoming. These methods are then, in the course of remembering their feasibility, applied to other fields. However, the selection effect of social rationalisation can only last if sanctions no longer protect an existing order or if the status quo is no longer preserved by a lack of alternatives.

In this context, the sub-process of differentiation represents an ever more progressing branching out and split of social fields of action. The development happening over time corresponds to a change from original unity to new diversity. Here, the knowledge of the possibility of differentiation can be proven as the memory-relevant selection mechanism. Like in the case of rationalisation, the structural aspect of memory consists of changing the given or of establishing the new; then what is memory-like is the principle of differentiation, coming along with the tendency of forgetting the previous unity or totality of a subject or of considering it to be outmoded. In this context, differentiating thought also includes the construction of the past, which also happens by way of memory, for also the differentiation principle generates a specific kind of memory while at the same time providing the precondition for an associative transfer to previously undifferentiated or only insufficiently differentiated fields.

The process of domestication describes the conquest of nature. Also, it can be stated that savageness and being subject to nature are forgotten. However, this kind of oblivion corresponds with the memory of natural disasters which have not yet been mastered or coped with. As soon as the damage caused by them has been repaired, the memory of disasters becomes a cultural

World War I. Concerning the latter development, she reaches back to Benjamin, among others, who diagnoses an inability to discuss the past for those returning from the war. 
achievement that rather seems to live on as a traumatic memory than as a reminder or warning.

The fourth sub-process of modernisation to be exemplarily mentioned here, the process of individualisation, is not understood as separation or solitariness but as attributing responsibility to the individual. ${ }^{46}$ Both the ambivalent process of empowering the individual - ambivalent because it is both liberating and overcharging - and the thus connected loss of the relevance of collectively binding guidelines for action can be memory-specifically analysed. The concerned individuals forget about their time of being institutionally embedded and from now on remember that social order can in principle be questioned.

The diagnosis of a time being accelerated consists of the assumption that the constant pushing through modern rationality comes along with saving time, which will necessarily result in, first of all, technological processes and social processes running faster. Accordingly, a shortening of presents is diagnosed, finding expression by an "increase of action and/or experience episodes per time unit" (Rosa, 2005, pp. 129-130). The result is a shortening and condensation of action episodes and the subjective perception of time becoming ever tighter (Nassehi, 2008, p. 13). As the diagnosis of the time emphasises the present becoming shorter, we may ask how significant then the reference to things past or to experience as an element of defining the current situation is. Does the present become shorter because an abundance of experiences needs more "space"? Or have the technology-induced constraints to reshape society grown so much that there is no more time for reflection? Thus, the shortening and condensation of action episodes would be a confirmation of Heidegger's assumptions on the oblivion of being or obliviousness.

Analysing acceleration in terms of memory theory means considering the technological possibility of saving time and its effects on everyday action. Then - also there - new relevance structures become apparent, which, as selection mechanisms, create precisely those processes as observed by the diagnosis of the time. This is, first of all, a technology-induced experience of condensation. Thus, the present is shortened not because of a simple topical focus, but it must first be understood as a technology-induced process that is external to consciousness. The social memory, we might assume by reaching back to Elena Esposito, is "telematically" changed by technological communications media to assume a change of the adjustment of perceived and processed information (Esposito, 2002). Against this background, selection mechanisms appear as being partly and increasingly determined by machines

46 On this see the debate in the wake of the individualisation thesis being newly formulated by Ulrich Beck (see Beck, 1994; Wohlrab-Sahr, 1997). 
so that in many cases, all that is left to "man" is reacting to the product of the no-longer-trivial machine. Similarly, the way or acceleration of movement influences the selectivity of perception. Thus, for example, movement via the automobile changes the perception of the topography of a place. When driving an automobile, one perceives the environment in a way that is different from being on foot; other aspects of the space one has been moving through remain as expressions. ${ }^{47}$ We might now assume that the technological developments work in favour of a changed adjustment of the experience of time, continuity, and perception - a development that might be connected to losing the aura, e. g. of a place. ${ }^{48}$

The process of globalisation refers to the dissolution of both cultural and national segregation as a result of new information technologies and transport means. ${ }^{49}$ We may consider the decline of the significance of spatial distances or national borders a crucial motif. In this context, globalisation appears as reversed differentiation, as existing separations are lifted. However, at the end of this process, there is no new unity but the plurality of the monopoly-based pushing through a particularly influential position. The memory-specific selection mechanism of this ambivalent process addresses the overcoming of existing limits, which is why we may also speak of delimitation, and it must be understood as a universal principle, as this principle, triggered by technological globalisation, can be transferred to different border regimes. Accordingly, the constitution of the present derives from reflecting on the successful overcoming of borders. Also, the delimitation principle may be a structural aspect of the social memory and generate specific ways of remembering, which again can be associated everywhere where existing borders are perceived as obstacles. ${ }^{50}$

47 Paul Connerton (2009) discusses this aspect as well as others as the oblivion phenomenon of modern society.

48 Felix Denschlag (2014) points out to the connections between Helmut Rosa's acceleration thesis and the works by Walter Benjamin.

49 As an example of the extensive debate see the overview by Ulrich Beck who characterised globality as follows: "Globality means that the unity of national state and national society comes unstuck; new relations of power and competition, conflict and intersection, take shape between, on the one hand, national states and actors, on the other hand, transnational actors, identities, social spaces, situations and processes." (Beck, 2000, p. 21)

50 Remarkable in this context is the debate on globalisation phenomena and transnational connection points for remembrance or remembrance regimes in the context of the process of European unification. There, the overcoming of connection points for remembrance or remembrance regimes limited to the national state results on the one hand in the search for new memory formats in the "old" sense of the national state (see the contributions in Feindt et al., 2014) and in considerations on kinds of a reflective particularism 


\subsubsection{Elements of a Sociological Concept of Memory}

When placing the collected elements of sociological theory next to each other, each element provides an explicit or implicit reference to or an interface for a theory of social memories or social remembrance. Also, this is done in contrast to equating memory with a material-spatial store of things past, as it is common in the cultural studies debate. Finally, it becomes evident that sociological thought is based on two motifs. One of them deals with why, against the background of open possibilities to act and interpret, only certain selections are made. The horizon of existing possibilities constitutes itself from a context of experience or stock of knowledge that is accessed according to rules or relevance criteria. The other motif comes from the separation of meaningful and non-meaningful practical, habitual or routine knowledge. Meaningful knowledge finds expression in communication about past events and is changed into an experience and transformed into action orientation through remembrance or comparison for congruence or coherence. On the other hand, nonmeaningful knowledge is kept effective by habitual behaviour without being explicitly referred to and perpetuated by constant repetition. Such a perpetual knowledge structure cannot be located outside the social - such as subjective consciousness or cultural artefacts. The stock of knowledge consists of a supraindividual stock of regulative, normative, connective and routine knowledge, the bearers of which are individual consciousnesses.

One further aspect is that the reference to the past or the path-dependency of the individual or collective action or behaviour may also always be powerinduced because of the social relation. Power may be habituated and then appears as an unquestioned, indeed almost unquestionable, opportunity, or it must, again and again, be legitimated by way of communicative acts which sometimes reach back to things past. This synthesis of aspects of social memories in the theoretical arsenal of sociological thought hints at the complicated ways in which meaningful and habitual influences affect the further development of social structures. It provides the foundation for systematising the dimensions of a concept of social memory, which is now going to happen. ${ }^{51}$

on the other (see Levy et al., 2011) which tolerates different and sometimes incompatible views at past events or integrates them as a new formatting principle of the collective memory.

51 Speaking of social memories in the plural and the next time of social memory in the singular is due to some theoretical undecidedness. If the concept of memory is implemented into different sociological theoretical systems, it must be decided case by case if the concept of memory must be expressed by the plural or the singular. In the context of the theory of social systems, for example, it seems to be appropriate to use the plural. If, 


\subsubsection{What is a Social Memory?}

The two motifs which have been worked out above have in common that they deal with social memory in terms of sociology; still, it is unclear how far these perspectives may be transferred to a concept of social memory or if they are different attempts at an explanation which cannot be further integrated is as yet unanswered. If that were the case, the social memory would stay as polyparadigmatic as sociological theory building as a whole. However, the variety of perspectives concerns primarily the various selection mechanisms of social memory and not its structure. Therefore, if we ask about a social-theoretical concept of memory in the following, we may expect to be informed about what is understood by social memory. It seems that questions about the "place" and "existence" of such a kind of memory are easily answered and combined. However, more disparate and thus also more difficult to bring together are the different principles of the "way of working" or the functionality of social memories - they change over history or, to have it in the language of knowledge sociology, they are "stationary". 52

The structure of the social memory - and here it looks obvious to construe an analogy of neuronal network structures and the culture-specific knowledge structure in the sense of the concepts of the social system, a society's stock of knowledge or the lifeworld - must be understood by connecting to scheme theories. What is understood as a cognitive scheme, on the one hand, appears - and here we may adopt the "sociologisation" of the scheme concept as performed by Schütz - as a socially constituted type or as a practical routine on the other. ${ }^{53}$ The function of memory is not the storing of information. Rather, it is that the memory organises the production of action- or behaviouroriented knowledge in the context of each current way of dealing with situations. In other words: social memory takes care that actors will behave as culture-specifically expected by combining their (subjective) perception with socially classified knowledge. Under this aspect, the social memory is equivalent to the individual memory, as, through lifelong learning processes, it has not stored but shaped and imprinted socially relevant interpretation patterns, types or routines of behaviour in the form of cognitive schemes. Beyond this

on the other hand, one is looking for universal principles of memory, rather the singular seems to be appropriate.

The logic - however not the empirical references or manifestations - of the argument to be developed is in line with Jan and Aleida Assmann's distinction of a storing memory and functional memory in the context of their theory of a cultural memory (see A. Assmann 2001).

53 Both the concept of scheme and his concept of social type are developed by Schütz (1967) in his book The phenomenology of the social world. 
equivalence, however, almost everything which, as a social experience, is based on socially constituted experience consists of learned "connectivities" or associations in the individual's brain structure. As social life/experience happens only mutually and not in an exclusively dyadic way; however, most of all among changing groups, the social creates a material imprint into the neuronal structure of the individual consciousness: successful behaviour is imprinted into schemes and scripts and can, by way of repetition and adjustment to each different situation, be ever more refined.

Another kind of "sociologisation" consists of detaching social memory from the subjects or individual consciousnesses. At this level, it is possible, with the help of the various variants of sociological structural analysis such as systems theory or the analysis of institutions, to reconstruct the intersubjectively valid classifications. Even then, social memory is still placed in the individual consciousness, as nowhere else knowledge as the network structure of associative and mutually associated schemes or types can be located. Accordingly, knowledge is located at or in consciousness systems; however, it develops from different interactions or communication structures. Thus, access to a so-called externalised or cultural memory - such as books or memorial sites - happens by making an informative communication experience that has already been caused by a selection achievement of the individual memory. In other words: looking for an expert term in an encyclopaedia would not mean having access to memory but would only be the purposeful search for a particular experience which then - as an experience - could modify a certain cognitive scheme. According to the terminology of the social sciences, rather than the concept of remembrance, the concept of "experience" must be associated with memory, as remembrance can always only be the current and perceptible result of reflecting on schemes of experience.

Time being an experiential aspect or a sub-scheme of its kind is inscribed into any scheme so that it is possible to construe a chronological distance to certain experiences. However, the time index is always applied to the scheme when modified by a recall. That is, time consciousness develops only by the process of remembering, which is why time must not be understood as a category of memory but rather as a product of remembering. ${ }^{54}$

54 Halbwachs's (1980) answer to Bloch's critique includes the example of a former student who, during conversation with his former teacher, reminds the latter to certain events which were meaningful for him. However, the teacher does remember neither these events nor when exactly they happened - in the course of the many generations of students passing by his schemes of experience were so much saturated that he was able to sink events which, from the points of view of his students, were significant and influential 
However, sociological theory offers taking up - implicitly or explicitly the issue of memory sometimes provide wildly divergent approaches which cannot easily be categorised under the same concept. If we are not satisfied with pointing out elements of a memory theory, it is advisable to look for the dimensions of social memory. Given the up to here considered approaches, three different trends may be pointed out; some of them are found not just in one but in several dimensions. Insofar, these dimensions serve as ideal-typical attempts at classification, by way of which the range of memory-specific connection points to the offer of sociological theories can be systematised. Thus, it seems obvious to reach back to already existing concepts of distinguishing types of memory as they are used in other contexts and by other disciplines. The dimensions - declarative-reflective (1), incorporated-practical (2) as well as objective-technical (3) - are laid out as structural dimensions, as they are always manifestations or "places" of memory providing an actor orienting in the present with an idea of reality which is no longer contingent but - given possibilities to interpret it - already selective.

The first dimension, based on psychological theories of knowledge and memory, refers to memory structures that can be derived from non-explicable reflective knowledge. Declarative-reflective is the term for all semantic knowledge contents which, as ideas or in the course of communication, can be experienced and transformed into experience. ${ }^{55}$ Purely semantic-cognitive knowledge theories are satisfied with this dimension ${ }^{56}$ which includes active, episodic and activatable knowledge. Habwachs pointed out that this kind of knowledge, although processed by a consciousness, cannot be changed by the latter into an experience without referring to a (social) environment. He explains that without inner or outer perception and associative connections to socially available frames, the subject is incapable of creating meaning.

The social institution par excellence which, like social memory, coordinates the attribution of meaning, is language. The symbolic representations of language - words and terms - are connected to cognitive schemes to identify and communicate a scheme of experience. At first, the scheme of experience stays to be exclusively a matter of the individual consciousness. Furthermore, through linguistic exchange or communication, it is turned into

in his perception routines. His schemes of experience were no longer modified, which is why also no new time index was inscribed into them.

55 Concerning some of its aspects, this concept is in line with what Giddens (1984, pp. 41-42) calls "discursive consciousness".

$5^{6} \quad$ This holds both for the philosophy of language connecting to Ludwig Wittgenstein (1999) in particular for the famous seventh sentence of his tractatus logico-philosophicus - and for approaches by way of analytical philosophy or also for the theory of social systems. 
an intersubjective experience that attributes symbols to the schemes, which then again serve as intersubjectively intelligible classifications. ${ }^{57}$ However, the connection of linguistic and written symbols to schemes of experience comes with interpretations that exclusively refer to social adjustment success.

Furthermore, associating certain experiences with a context of meaning or discourse may happen as a pattern of cognitive processing or as a chain of associations. Such a view is also in line with communications theory the theory of social systems is based on. The idea of a subject or subjective consciousness is excluded - however, the process of constituting a system through follow-up communication (autopoiesis) is based on a structure of the semantic association. This may also be transferred to the concept of the narrative or narration. Accordingly, narrations may be grasped as network structures on the one hand and as schematised experiential contexts of their kind on the other. Then the identity of the individual appears as a schematized self-narration serving for providing orientation in certain situations. The situation is similar in the case of the different kinds of collective identity. Also, the group members are provided with symbolically generalised and schematized patterns of their history of attributions that have been imprinted into the group in the course of mediate and immediate collective experiences. In any case, however, the emphasis is on linguistic-symbolic representations of cognitive schemes. Accordingly, the declarative-reflective memory is a socially constituted, while at the same time cognitively schematised association structure serving for finding orientation in a current situation. This happens by reaching back to experiences as a subjectively construed past and the thus connected creation of (inter-) subjective legitimation for the following action. Action - as we may say by connecting to Schütz - is meaningful as it is drafted or pre-remembered based on memory. Thus, the consciousness "reflects on" the field of the theoretically and thus semantically concluded knowledge - which, due to the time indexes also inscribed into the schemes of experience, is experienced as one's own layered past re-experienced.

The social-scientific reconstruction of the declarative-reflective social memory makes use of the analysis of symbolic manifestations as it is performed in the context of knowledge-sociological discourse analyses ${ }^{58}$, analyses of interpretation patterns or narration analyses, such as in the context of biography

57 On this, once again see the scheme theory unfolded by Schütz (1967).

58 Referring to the special case of the knowledge-sociological discourse analysis (Keller 2005 ) is due to the programmatic connection to the present of the knowledge-sociological approach while delimiting from discourse analyses which are based e. g. on historical sources. 
research or the research of collective identity with the help of empirical methods. The result is the reconstruction of semantically generated patterns of meaningfulness that may claim to be intersubjectively or "objectively" valid and are to be found, e. g., where social groups assure themselves about their cohesion or where traditions as past-related narrations - such as the narration of the nation or other founding myths of a collective - are purposefully invented to give reason to order-relevant ways of behaviour.

The second dimension of social memory refers to concepts formulated by explicitly delimiting from cognition-related knowledge theories. These are perspectives covering phenomena such as routine knowledge, habitual knowledge, implicit or non-declarative knowledge. Thus, incorporated-practical knowledge shall be the term for that kind of knowledge which, like declarativereflective knowledge, is the precondition for successfully coping with a situation but is not attributed with a specific meaning or need not become an element of defining a situation or of drafting an action. ${ }^{59}$ As much of everyday life is coped with by way of routine, this is possibly by far the widest field of practice-relevant knowledge. Walking, swimming, riding the bicycle, the way to work - all these are activities which once have been learned with more or fewer difficulties. However, in the course of making regular use of them, they have been incorporated to such a degree that they can be performed without the reflecting consciousness being involved. On the contrary, their smooth operation may even be disturbed by immediately activating the consciousness. There is no doubt that this is a kind of knowledge that is based on structure or the structuring and selection function of memory. This kind of knowledge is socially or culturally communicated since even basic movements have been learned through imitation. Moreover, as even allegedly automatized behaviour is theoretically contingent, we may assume that vast parts of this knowledge are culture-specifically influenced.

Given the individual, we must assume that incorporating such a kind of practical knowledge also happens according to the schematisation principle. However, here it is natural to use the concept of the script as it is common in psychology for processes. Via the script, certain movements and perception sequences are defined. Like the scheme, also the script can be shaped. As soon as a disturbance occurs, there is a functionality assessment - entirely automatically and in passing - due to which it is updated and "rewritten".

These scripts may be incorporated because sometimes they are habitually imprinted into the physiognomy and the movements of the young

59 For this memory-relevant aspect Giddens (1984, p. 41) uses the term "practical consciousness". 
person - skilfully playing a musical instrument is only possible by way of appropriately training the muscles and switching off any reflection on each individual movement. Also, connecting such a kind of habitual incorporated-practical knowledge to the relevancies of a social field goes back to Bourdieu. The social is imprinted into the individual by youth-specific differences in typical competition situations the individual is frequently confronted. Accordingly, the class habitus is an empirical indication of social belonging and social power relations. Thus, the incorporated-practical social memory has its place inside the individual, in which the subject's or the consciousness's reflecting must be mostly ignored. However, this kind of memory is also socially constituted, as there are only a few reasons for internalising and incorporating scripts not triggered by the model of other relevant, culture-specific ways of behaviour.

Furthermore, there are routinized relationships in the sense of collective behaviour, which are based on path-dependencies without necessarily being legitimated by individuals or by the group giving reasons to them. Among them, there are habitual movements in the context of family relations as well as expressions of sympathy or triumph. However, empirically working out such kinds of memory cannot be based on semantic material and thus depends on data collected by way of observation or on comparisons. Asking the person under observation why he/she shows a particular way of behaviour is pointless right from the beginning, as it would reveal rationalisations in the sense of declarative-reflective patterns of reason-giving, which would rather be an obstacle for an understanding reconstruction of behaviour. The analysis of this kind of social memory must aim at the practices themselves, insofar as by practices - or traditions of acting - we mean processes of social behaviour which mostly evade reason-giving by individuals.

Objects or artefacts represent an "objective" or objectivised reality from which results in not only an immediate need for action but also provides prior orientation. Many objects the individual encounters are more or less familiar to him/her because they are connected to specific schemes of experience. The brain associatively processes these impressions by comparing stimulus information with corresponding patterns of processing. The selection principle is that of similarity. Insofar, we might understand objects as the bearers of social memory, as usually they refer to socially habituated ways of using them and meaning contexts. Schematisations - that is, the experiences a subject has made with objects - are highly socially communicated. Schemes do not only consist of cognitive processing structures connected to a standard expectation of usefulness. They also result from a wide field of socially communicated meanings that are not necessarily based on immediate experience ${ }^{60}$ 
connected to possible adjustments to social expectation horizons. Given the memory dimension of objects, this raises a question: on the one hand, the orientation achievement induced by objects refers to declarative-reflective knowledge developing from immediate and mediate-socialisation experiences and stored via the experience scheme - in the sense of cognitive-neuronal structures of the processing of information. On the other hand, the routine use of objects may also be interpreted in the context of incorporated practical knowledge. Given these objections, the memory of objects must be considered an offspring of the other two kinds of social memory. ${ }^{61}$

Given the development of communications and information technology, however, exceptional cases can be identified where memory aspects can partly be separated from the consciousness by which they have been initially constituted. As soon as a machine generates information the related person does not expect, schemes and scripts become irritated. Telematic information as knowledge content, as it is produced, e. g. through data mining, is capable of determining a selectivity of its kind (Esposito, 2002; Hagen, 2011). Only in such a case, a technological process, such as in the context of a network of actors (Latour, 1993), becomes a quasi-independent key player, and only then it may be justified to speak of an objective-technical memory. Nevertheless, it is still a special kind of social memory, as it is constituted in the mode of relation: under specific conditions, even machine-produced content may provide the individual with orientation in specific situations and may result in action plans. A structurally similar situation results from the phenomenon of the unexpected incident. Also then a technological device produces unexpected information and confronts the individuals dealing with it with interpretation and possibly also action problems. However, in contrast to the non-trivial machine, when the production of unexpected information is foreseeable, the incident is an exception the way of coping, which leads to a modification of the scheme or script connected to the artefact.

Many of the memory approaches negotiated so far may be systematised according to these three kinds - declarative-reflective, incorporated-practical, and objective-technical. However, it has already been pointed out that some evade this classification insofar as it may be attributed to several dimensions of memory - such as the sociological concept of institution. On the one hand, it appears as explicable structural and regulation knowledge which becomes manifest solely in the connected system of sanctions. On the other hand, the sociology of knowledge understands institution to be the objectification of

61 Paul Connerton (2009) discusses the memory nature of the medieval urban structure in the sense of orientation which the visitor can derive from exposed buildings. However, it is always left to the orienting social entity how it deals with the topic of place. 
routine knowledge. Then the explicability of the institution is only based on myths or rationalisations.

A second concept that evades any clear attribution is the connection of subjective experiential context and social stock of knowledge. The subjective experiential context is a combination of declarative-reflective and incorporated-practical memory, as it is based on schemes or scripts of experience and habitual knowledge. It is the basis and starting point for intersubjective understanding, from which there derive mutual classifications which are usually connected to gestural or linguistic representations. The total of these classifications within one cultural space constitutes the social stock of knowledge, which includes incorporated-practical memory, due to routines and habits of everyday-unquestioned knowledge being sedimented. Thus, the social stock of knowledge would be suitable as a memory equivalent. Although Schütz and Luckmann phrased it in a more differentiated way, the formulation suggests spatial storing. Accordingly, it seems more understandable to associate imprinting and selection with memory instead of a stock. ${ }^{62}$

The third concept which cannot easily be attributed to one of the three dimensions is that of the frame. Indeed, Halbwachs's frame concept may be understood against the background of declarative-reflective memory, and it may be attributed to the different, interrelated schemes of experience - in the case of family, religion or social structure, this would be unproblematic. As concerns the second aspect, addressing the basic social categories of space, time or language, the latter may be attributed to the declarative-reflective realm. Halbwachs's concept of collective time refers to conventions of time as a social frame for subjective time consciousness. However, in the context of his timetheoretical considerations, Halbwachs starts from Bergson's concept of inherent continuity as a dimension of experience that cannot wholly be put into words. The situation is similar when it comes to his concept of spatial framing, which, after all, constitutes from the schematisation of modes of exploring space, which depends on being updated at regular intervals. However, the undoubtedly cognitive classification of spatial framing shows a not circumventably practical aspect. Space is not explored in a cognitive-understanding way, but it is paced, experienced and thus incorporated.

The frame concept can be crucially extended by including the frame of the interaction order in the sense of Goffman. However, not even this solves the problem of a clear attribution to one of the dimensions. For, even after

62 Thus seen, the concept of archive, where storing and cataloguing are brought together, seems to be appropriate. However, the concept of archive suggests material storing, which way the stock of routines and habits would be left out of consideration. 
Halbwachs's frame concept has been completed by Goffman's, it is an open question if it is first of all declarative-reflective stocks of knowledge which provide each current and mutually designated definition of a situation by the individuals with orientation or if the perception of and dealing with frames happen intuitively or routinized. Thus, the here involved social memory is both of a declarative-reflective and an incorporated-practical nature. The frame is - similar to the habitus concept, which must also be clearly grasped as an incorporated-practical memory - both structured and a guideline for structuring. ${ }^{63}$

In view of sociological structural concepts, it has become apparent that they address different dimensions of a social concept of memory. However, they consider only sub-aspects. Among those concepts that may be referred to several dimensions, no concept could be identified that takes the orientation of acting by reaching back to the past into consideration to such a degree as social memory. Such a concept of memory has no "reality" of its own - it is "located" both in the consciousnesses and bodies of the individuals and habituated social orders, arrangements of behaviour and relations. There it is represented by associated and associative scripts and schemes. Thus, the memory-theoretical deficit of sociology is that it does not comprehensively reconstruct and examine all aspects of memory as abstract contexts of meaning and structure.

The structural aspect of social memory as a symbolic and practical order comes along with what initiates the addressing of certain schemes. Memory constitutes itself by schemes, scripts, and types; however, we must assume a selection equally as not all of them are recalled equally every moment. Thus, whereas given the question about memory structure, we may understand the social function of memory as being similar to the individual's brain. Because of the problem of selectivity, we can identify another function that has occasionally been described here as "true" memory. As concerns social memory, it may be stated that precisely the specific selection function is "objectively" determined by social learning in groups and by the thus developed cultures. ${ }^{64}$

Then, however, to such an objectivist view of the selectivity of social memory - or, if like with the theory of social systems, networks of actors, discourses, or realms of the social, we assume several memories - adds the analysis focus of the analysis the memory researcher. Then an analysis of the

63 On the parallels between these two concepts see Herbert Willems (1997).

64 Against the background of group-specific selectivity it becomes obvious that, no matter in which way we might read Luhmann (1995), culture cannot be anything else than a social memory. 
relevance structure ${ }^{65}$ of the social memory provides analytical access to this selectivity which itself, on the one hand, already controls perception by reaching back to habituated perception routines and meaning-attributing or reasongiving interpretation on the other.

In the context of the declarative-reflective dimension of memory, we must assume ways of selecting which refer to communication selectivity. In this context, the focus is always on which elements of knowledge accessible to consciousness are reached back to for each current definition of a situation. ${ }^{66}$ In view of Halbwachs's frame theory, one gets the impression that, after all, the hints at the society that exist in the environment also provide the stimulations preparing the cognitive act. The individual perceives things that it interprets by help of those schemes as offered by and grown in social adjustment processes. Vice versa that what cannot be attributed to any appropriate scheme cannot be understood - and usually, it does not become relevant, so it is not perceived and not remembered. Yet, it is unanswered which one of the frames existing in many situations is concise enough to be perceived as relevant not only by one but by several individuals. One possible explanation is the assumption - on which perhaps also Halbwachs based his considerations - that there must be group-related relevance structures attributing higher priority or conciseness to certain frames in any culture. When it comes to European societies of the first quarter of the 2oth century, this holds for both levels of the frame system. Accordingly, from a sociological-social-structural perspective, family, religion, and social status are as essential as the basic categories of time, space, and semantics from an epistemic one.

Further aspects of selectivity are provided by the social-scientific discourse theory and the theory of social systems. Discourse theory answers the question about the perception problem only indirectly. It is much more specific when it comes to analysing the circumstances under which things are considered relevant. The linguistic order of things is power-induced, so a particular meaning is inscribed into their names and a structure of importance. This is boiled down best by the concept of the hegemonic discourse. ${ }^{67}$ There the selection

65 It is no coincidence that Schütz completes his spatially connoted concept of the storing of the social stock of knowledge by the concept of the relevance structure (see Schütz, 1971; Schütz \& Luckmann, 1973), to be able to take the problem of selectively reaching back to knowledge into account.

66 Here, the concept of defining situations according to William I. und Dorothy S. Thomas (1973) seems to be imprecise, as it does not distinguish between behaviour and action or between automatic-instinctive and meaningful action.

67 By a hegemonial discourse, Siegfried Jäger (2011) understands a ruling or pedominant discourse. 
performance of the social memory, that is, the relevance structure determined by the power structure is so predominant that any emerging object can only be interpreted in a certain way if specific power interests communicate it. This may go as far as to make it impossible that even "authentic" memories, based on individual experiences but not being in line with the discourse, are articulated without the danger of being sanctioned. ${ }^{68}$

The selection mechanism has become very accentuated and elaborated in the context of the theory of social systems. As systems are autopoietically constituted from interrelated or interconnected communications, it must be constantly assessed which communication belongs to the system and which not (Luhmann, 2012; Dimbath, 2011a). This assessment, which necessarily must refer to the structure - or memory - of the system, happens along the systemspecific binary coding.

Thus, the hints at a declarative-reflective social memory given by socialtheoretical positions allow for concluding different selection mechanisms. On the one hand, these conclude from culturally-evolutionarily "grown" relevancies whose orders are immediately action-guiding for any individual. Not only meaning-creating remembering as a phenomenon whose occurrence Halbwachs attempts to understand by reaching back to the motif of collective memory, but any definition of the present refers to social patterns of order right from the beginning when current perception, as well as the processing of what is perceived, are organised. Then this classification also determines the view of other perceptions and how to deal communicatively with them. Although it does without a processing subject, a similar motif is found by a more topical manifestation, with the selectivity of system memory. The selection principle may vary when it comes to determining relevance due to functional differentiation. In both cases, however, the selection principle is based on the motif of evolutionary adjustment or evolutionary development. As it happens slowly, the involved individuals or most observers are not immediately aware of the change of selectivity. In contrast, the discourse-theoretical position is not satisfied with assuming a "primordialism" of whatever kind and explicitly emphasises the relevance structure being shaped by power interests. Admittedly, here one does not assume any rule going beyond particular interests; instead, it is about securing power or later affirmation that is supposed to secure the current situation by way of selection guidelines. Thus what is left - this is how we may sum up the selectivity trends in the context of the

68 Relevant hints are to be found with remembrance cultures, such as the socially developing memories of war children (see e. g. Heinlein, 2010). 
declarative-reflective social memory - are only two basic lines. The selectivity of memory refers to the remembering situative attribution of meaning:

Firstly, it refers to an evolutionarily grown social order. This allows for dealing with the given and may perhaps result in changing it. However, it usually only results in structural maintenance and perpetuating the structure.

Secondly, it refers to an order which is determined by power interests. Then it seems as if the goal of maintaining and continuing certain structures is instead achieved by way of radicalising this order. Also, only in exceptional cases, interpretation happens independently of selectivity. Other situations requiring attribution of meaning are right from the beginning considered from the previously taken point of view. Due to the existing being meaningfully rooted in an ordered past, the world is considered to be "in order". ${ }^{69}$ However, by researching modernisation processes and social change, sociology has worked out a position from where the required reference to the past is systematically questioned.

As concerns the incorporated-practical dimension of social memory, selectivity must be grasped by way of routines of the behavioural disposition. The stability of these routines is due to the practical action context usually not being disturbed. ${ }^{70}$

The concept of habitus, oriented at the competition structure of the social realm, makes it evident that past-induced selectivity is different according to the position of the individual. Accordingly, it is determined on the one hand by the exchange relationships relevant for the field and by successful longterm, habitual action strategies as well as ways of behaviour and presentation on the other. Although Bourdieu points out that the rules of the field may be changed (Bourdieu \& Wacquant, 1992, p. 99), habitus still is attributed much persistence. As the kinds of habitus develop by way of interactions with the exchange practices of the field, also there we may assume a quasi-evolutionary constitution. Selectivity is indeed habitus-specific - change can be experienced only if an actor has not been in the field for some time and presents an outdated habitus ${ }^{71}$ - however it is not necessarily connected to a powermaintaining or status-maintaining tendency. Rather, this might be based on a master-servant dynamic, in the context of which the power structure has two

69 Schütz (1967) bases his sociality theory on this fundamental insight, without explicitly calling it a memory theory. The idealisations of I-can-do-it-again-and-again as well as and-so-on appear as necessary assumptions for basic trust in the world being organized.

70 As soon as this is the case, however, usually there does not happen unreflected relearning but at first a declarative-reflective interpretation.

71 A short description of the hysteresis effect, by way of which the persistence of habitus is described, is given by Peter Wehling (2011b, p. 176). 
sides that must constantly be balanced. Thus, the selection principle refers to respectively valid rules of behaviour of social classes, strata or milieus. This becomes evident for everyday life, for example, by phrases such as "somebody cannot escape his/her skin", "cannot swallow his/her own pride", or otherwise would have to behave "contrary to his/her nature".

In the context of the incorporated-practical dimension, memory-specific selectivity does not pose any orientation problem. Questions such as "What is going on here?" or "What am I going to do?" are irrelevant as long as there is no irritation. According to established and typical, situations are gone through practically due to the social disposition - routines. According to Bourdieu, coping with basically contingent situations happens according to those routines as having evolutionarily developed in a field for a specific type of actor. If we leave away the field-relatedness emphasized by Bourdieu, after all, there only remains the scheme-theoretically explained selection figure - a selection by way of associating with similar schemes or routines. ${ }^{72}$

That routine-guided behaviour is based on past experiences can hardly be doubted. According to socially constituted imprinting, the memory "invisibly" coordinates intuitively appropriate processes. Now, however, remembrance has been attributed exclusively to the realm of the declarative-reflective memory aspect. In everyday language, however, we also find a way of understanding remembrance which rather aims at incorporation. There is no doubt that, when it comes to routines, the selectivity of memory refers to something "inherent" - to schemes and scripts. Notwithstanding the clear idea that the body, when falling into the water, "automatically" re-calls swimming movements, remembrance would be equated with memory, resulting in a loss of precision. Thus, as concerns the incorporated-practical dimension of memory, it seems to be rather fruitful to give up on the concept of remembrance. In other words: remembering as a process of consciousness remains reserved to the declarative-reflective dimension.

In the context of the objective-technical dimension of the social memory, it has already been pointed out that this is a special or mixed type of the two other dimensions. However, it seems as if this third dimension of memory shows a particular kind of selectivity. Most of all, in the context of communications and information technologies, it is possible to reach back to information in a way that wilfully generates information. In the course of this, the technological device copies - for the time being in a very limited way - the constructive selection work of the memory and combines it with algorithmic

72 Here, further stimulations may be expected from reaching back to Gabriel Tarde's theory of attracting and imitating similarities (2013). 
procedures and technological-instrumental associations. In exceptional cases, it may then be that such a technological actant is attributed to the status of an actor within a network. In such a case, the social memory is provided with technological selectivity, resulting in the network orienting its behaviour at technologically constituted selections. ${ }^{73}$ The social memory, we may now state, is a socially constituted structure of reaching back to things past. However, the past is not available in the form of stored - in whatever way - information but as an always present structure of schemes and scripts which in the realm of intersubjectivity and as type and practices may constitute mutual horizons of expectation. The primary function of this kind of memory is the selection of ways of behaviour in respect of each current situation. The rules, according to which this selection happens, may depend on different structural dimensions described as declarative-reflective, as incorporated-practical and objective-technical. Given existing sociological theories, four kinds of selectivity can be identified: the evolutionary kind, the interest-guided kind, the fieldrelative kind, and the telematic kind. After these systematising considerations on the concept of social memory, it should now be possible to analyse the conditions for social oblivion in more detail.

\subsection{Oblivion Motifs in Sociological Theory}

So far, the few explicit examples of discussing the phenomenon of "oblivion" to be found in sociological literature have been excluded from our elaborations. In the following, we will pursue both explicit and implicit oblivion motifs found in sociological literature. ${ }^{74}$ All the already discussed sociological concepts provide answers to how the thus described social structures are

73 That such a selection refers to the past appears as a necessary precondition, as hardly any social entity could be satisfied with ahistorical, or better: coincidentally provided, information. The processing of data collected in the past appears as a precondition for an objectivist-technical social memory. It is remarkable, however, that it seems as if here no reaching back to a meaningful past seems to have happened - this way the construct nature of remembering is emphasized even more. At the same time, however, reaching back to databases may be subject to delete functions, so that technology contributes to forgetting information which may be expected. Astonishment about allegedly unimportant data being stored for a long time corresponds to astonishment about the routine destruction of allegedly important information - although usually this happens only with hindsight. On the problems of automatized memories see Viktor Mayer-Schönberger (2009; 2011), Christine Plass (2005) or Oliver Dimbath (2008).

74 A first inspection of the oblivion-theoretical potential of the canon of sociological theories has recently been initiated by Oliver Dimbath and Peter Wehling (2011). The following 
maintained or changed in the long run. Each "and-so-on" presupposes reaching back to the past, and each modification - no matter how minor it may be - of resulting activities is accompanied by aspects of oblivion. Like the previous ones, the following considerations will be about a conceptual overview or stock-taking. They will be oriented at structuring the sociological field of work alongside social theory questions, social theory in the context of social order, and theories of social change. By doing so, we will be satisfied with collecting explicit elements of a sociological theory of oblivion. In the context of sociological considerations, the distinction made so far between forgotten social entities ("subject perspective") and forgotten objects ("object perspective") seems to be irrelevant, insofar as it is stated that objects may fall into oblivion. Then, however, the research interest will be in the social conditions of oblivion; in other words: the forgotten object is of interest only concerning its trace, which will then be the subject of communication.

By looking for indications of oblivion in the sociological debate, the research interest will at first be in this topic and not in the concepts and theories probably implying this phenomenon. In this context, it must be taken into consideration that sociological theories (do not) negotiate in particular the issue of forgetfulness as a feature of social entities in each different ways, each according to dealing with oblivion in the context of sociality and social theory, of the theory of society or social change.

Stocktaking in sociology is at first interested in sociological statements on the socially communicated possibility of the forgetfulness of the - always socially integrated - individual. In particular, the considerations by Alfred Schütz, which can be completed by theoretical aspects from the field of the sociology of the body, will be in focus; Bourdieu's practice theory will be crucial in this context. Then, and given social orders, it will be about the normativeconventional organisation of oblivion in the context of social relations. There, the analyses by Halbwachs, the sociological interpretation of the discourse theory according to Foucault as well as stimulations by the theory of social systems according to Luhmann will have to be taken into consideration. Finally, we are going to ask about the historically changed possibilities of oblivion, in the context of which Elena Esposito's systems-theoretically inspired analyses will be taken into consideration, just like Paul Connerton's modernisationtheoretical considerations and the analyses made by Rainer Keller from the point of view of the knowledge-theoretical discourse analysis.

considerations pursue a thus based claim to systematization, however at the same time they are more selective. 


\subsubsection{Social Relations}

Research work in the field of social theory as a theory of social or sociality deals with answering how social relations are possible. Considerations in this concern start with the capability of the individual (ego) to relate to others (alter), communicate with them, and raise mutual expectations, which, after all, provide the basis of every social order. A phenomenological perspective much determines this approach; nevertheless, approaches from other thought traditions provide answers concerning this field. Examples are methodological individualism in the sense of sociological action theories or the theory of symbolic interaction and structural theories such as the theory of social systems. However, all these approaches must be extended by assuming that perception is always socially shaped or co-determined. The meaning a consciousness attributes to its perception is the result of subjective interpretation. The constitution of meaning does not require all information perceived in a given situation but only a selection depending on socialisation or experience. In other words: each subject generates meaning based on selections which can be reconstructed by their relations to relevance structures.

Up to here, this assumption has only concerned the individual. Because of the individual's relations to others, the assumption results in the problem of double contingency: if the meaning is exclusively a product of selective perception, the possibility of mutual understanding must be fundamentally put into question. The individual consciousness produces meaning - both when attempting to understand the behaviour of its counterpart and when planning its actions - by reaching back to past experiences. Also, these are addressed in a highly selective way, however. What is provided with meaning by humans, what these humans believe in doing, intending and understanding while doing so can be reconstructed given their biographically supported definition of each situation. However, an alternative approach is to understand which experiences or knowledge contents they must have ignored or forgotten to come to their specific definition of a situation.

The most elaborated offer of social theory to answer this problem is found in the works on the structures of the lifeworld presented by Schütz and continued by Thomas Luckmann (Schütz \& Luckmann, 1973). In the context of social behaviour, however, we may as well exclude the conscious attribution of meaning and ask about automatisms, routines, or practices that determine the individual's actions. Schütz and Luckmann also make suggestions in this concern; however, some considerations further specify this solution in sociology. In particular, the theory of practice, which was made available for sociology by Pierre Bourdieu, provides answers. Thus, in the context of a social-theoretical analysis of social oblivion, the social-phenomenological approach, according 
to Schütz, will be analysed first. Then Bourdieu's contribution will be analysed for explicit and implicit connection points for a knowledge-sociological theory of oblivion.

\subsubsection{The Relevance Problem as a Selection Principle in Alfred Schütz}

In his works, Alfred Schütz is primarily interested in understanding the structuring of the social, which is communicated through subjective meaning. Connecting to Max Weber's considerations on meaning-communicated reason-giving for (social) action, his work is characterised by the attempt to reconstruct social order or social structure, starting from the individual's possibilities to attribute meaning or the problem of mutual understanding. However, as subjective meaning is not based on transcendental criteria, Schütz works out a complex theory of the piling up of structures of meaning, starting from individual experience via the idea of an experiential context as far as to the social stock of knowledge. ${ }^{75}$ In other words: the individual generates meaning from the history of his/her experiences, which is why Schütz must integrate concepts of time, past and remembrance into his theory. In the following, some of these aspects of Schütz's action theory will be presented as far as they deal with these problems (1). This is followed by an inspection of the ways and contexts in which Schütz makes use of oblivion for his architecture of theories. As Schütz explicitly mentions the term "forget" several times, it will at least be possible to collect and assess indications of a concept of oblivion (2). Then by a final step, we will search Schütz's works for a social-phenomenologicalknowledge-theoretical theory of oblivion (3).

The starting point for Schütz's thought is the consciousness of the individual. Before turning towards the analysis of the social, Schütz clarifies the individual preconditions for the perception of inherent and environmental objects. Bergson's philosophy of life, particularly the latter's concept of inherent continuity and his concept of memory, ${ }^{76}$ provides answers to how individuals develop a time consciousness by looking back to past experience. Connecting to Bergson's critique of a spatial-chronological concept of experience as the basis for scientific understanding, Schütz adopts the idea that an analysis of consciousness and perception must start experiencing the world. In contrast to general experience, he states that individual experience is not

75 On this see most of all the fundamental work The Phenomenology of the Social World (Schütz, 1967), where he attempts to substantiate the concept of "social action" in Max Weber.

76 These considerations by Schütz were comprehensively worked out in his Phenomenology (1967) - however many of these motifs are also to be found in the manuscripts on Life Forms and Meaning Structure (Schütz, 1982). 
yet distorted by theoretical reflection processes and spatial and chronological classification. At quite a fundamental level of consciousness, says Schütz, there is neither an idea of space nor time. This concludes from Bergson's concept of inherent continuity (durée). Continuity consists of continuous becoming and unbecoming and is thus irreversible in principle. As concerns, pure continuity, at the first level of consciousness experience is followed by experience, without the thinking consciousness being able to establish explicit connections. The only thing these experiences have in common is the fact that they are perceived by consciousness. Only at the next level, which Schütz calls continuity gifted with memory, the individual experiences are related to each other - only by remembering a past, it is possible to identify the qualitatively different now. Thus, memory must have recorded aspects of past experience, of what has been experienced, to make them available for the comparison with new perception experiences: it creates a connection between a just recently recorded perception image and a corresponding, imaginative idea of a past experience which has been stored by the memory.

At the same time, the memory itself is subject to continuity by adding current impressions to each memory image, thus continuously renewing them. Thus, by memory, we must understand the reproduction of a memory image that is emphasised in continuity. The memory connecting the already existing memory image to new perceptions allows for establishing connections that might be called images of meaning - it thus creates meaning.

The as-yet described processes have been rather inherent; only through a second visualisation is it possible for the individual to perceive objects in time and space. This way now, the inherent image of continuity gifted with memory is connected to the physical emotion, which is current at the moment of processing it. By the body's movement, action, now the I makes itself capable of experiencing space by understanding the latter's continuity as time. The continuous, manifold succession is transformed into a discontinuous and homogeneous simultaneity. Now action appears as a reinterpretation of an inherent volitional act within an outside space. However, this is only possible if the I understands a running movement as a finished movement. "The reinterpretation of ongoing into finished movement, which occurs in and through the acting I, destroys inner duration. It breaks through it and simultaneously renews it in a different and higher sense" (Schütz, 1982, p. 105).

However, for the I, the new kind of movement no longer consists of the becoming movement but of the unbecoming movement; that is, past movement. Every action, every movement in space, every object can be imagined by the acting I only based on the unbecoming.

However, there are also things the I is capable of experiencing without sensual stimulation or movements: the fellow humans. Any relation to the you is 
only possible if the I assumes that it is provided with a basically similar way of dealing with its continuity. The consequence - and also this is only possible by way of memory - is the assumption that the experience made by the I and triggered by the you comes from the you. I assumes to be understood by you while at the same time assuming to basically be capable of understanding you. Thus, Schütz names a basic precondition for any kind of social memory in passing.

These consciousness-theoretical primary considerations, inspired first of all by Bergson, are the starting point for Schütz's action theory. ${ }^{77}$ Also there, he introduces the concept of continuity first and points out the difference between running action and completed action. The I turning back to its continuity being finished, which is only possible by giving up on experiencing the current continuity, is what Schütz calls reflection.

For only the fact that an earlier phase preceded this Now and Thus makes the Now to be Thus, and that earlier phase which constitutes the Now is given to me in this Now in the mode of remembrance (Erinnerung). The awareness of the experience in the pure stream of duration is changed at every moment into remembered having-just-been-thus; it is the remembering which lifts the experience out of the irreversible stream of duration and thus modifies the awareness, making it a remembrance. (Schütz, 1967, p. 47)

In Husserl's work, Schütz finds similar solutions that he believes to be more promising. Accordingly, he states that Bergson's distinction between running and finished continuity is given more thorough reason by Husserl's analysis of the inherent consciousness of time. Husserl's distinction between primary memory in the sense of a still lasting consciousness of the original retention and secondary memory as a reproduced re-memory completes the understanding of continuity gifted with memory. On the other hand, his explanation of the perception of movement as permanently establishing things as-beingnow is similar to Bergson's elaborations on the updating of memory images. Whereas the retentions fade away or are forgotten with growing distance, the reproductive re-memory can be updated by immediate access or reproduction. The first impression - Schütz calls it the original impression - is subject to continuous adumbration, consisting of already the memory of the original impression changing it as a memory image. In other words: the original impression is enriched with a memory of the memory and so on. In the case of re-memory,

77 It is perhaps underestimated how much Schütz's thought was influenced by Bergson's philosophy, as Schütz, after having exploited Husserl's works for himself, "changed over" to a theoretical perspective which was in many aspects similar and sometimes even more precise. For analyzing Schütz as a sociologist of memory, however, the appreciation of his Bergson period seems to be very important. 
on the other hand, there is no reaching back to original consciousness. It is always unclear and has thus no evidence of absolute certainty.

Apart from original retention and reproductive re-memory, Schütz also adopts the context of protention from Husserl. Protentions are empty expectations that are derived from memory but aim at the future:

Therefore, what was empty expectation for the actor is either fulfilled or unfulfilled expectation for him who remembers. That which, for the actor, points from the present into the future, for him who is rememberingpoints from the past to the present moment, while still retaining the temporal character of the future. The intentional glance, then, is concerned only with the act (Handlung), not with the action (Handeln); and acts are always fullfilled, never empty, protentions. (Schütz, 1967, p. 59)

Any purposeful shaping of the world by the I must before be imagined based on memories. Thus, memory is reflected towards the future by the present. In the course of this, protentions develop as empty expectations, which are then filled in the course of action. We may thus state that purposeful action as fictitiously completed action must always be drafted or pre-remembered.

As concerns the relation of I and you, the same holds as for the considerations connecting to Bergson. Also, an action referring to a you, if it is supposed to affect the you, is drafted and thus based on memory. However, it must be based on the uncertain assumption that the you refers to preconditions that are similar to those the I refers to. ${ }^{78}$ Thus, social acting happens according to an attitude Schütz calls modulation by others. One must guess the course of the continuity of the you, by making the consciousness experiences probably happening there the precondition for one's plan for action one has imagined by the pre-memory.

By his memory-based considerations, Schütz demonstrates that sociality comes neither from any "natural" drive nor from any spontaneous, rational decision but that the social relation consists much of actions drafted by reaching back to experiences. These action plans are based on adopting the assumed perception of the other, which is a fundamental element of one's plan for social action. All this, however, is based on one's continuity gifted with memory whose corresponding consciousness experiences are assumed to be similar to those of the other. No matter if I and you understand each other at all, they will - insofar as they discover any opportunity based on similarity - start to act by referring to each other. The observed results create memory images of this more or less successful relation from which expectations result concerning

78 This corresponds with Schütz's (1967) general thesis of the alter-ego. 
further pre-remembered drafts. From the dyadic relation between I and you, it is now possible to conclude the development of social relations structures.

The subjective memory images may also be understood as schemes of experience. Through the social relation, they are provided with a particular nature, as some aspects of memory images developing from a relation can be fixed as again and again applicable aspects of successful communication. It is possible to derive the development of a system of symbols - such as a common language - just like a complex structure of mutual expectations by way of which social relations are provided with a reliable order. In particular, by tradition, passing on such stocks of knowledge to follow-up generations, the intersubjective classifications, which are initially based on subjective experiences, assume the nature of unquestionable objectivity. Thus, later generations need not make all basic experiences, as from earliest childhood, they are provided with a culturally grown linguistic-conceptual and rules-based behaviour.

However, knowledge may also be lost. What Schütz calls "restorable knowledge" is some earlier knowledge that has been lost or eclipsed by other knowledge (Schütz \& Luckmann, 1973, p. 215). On the one hand, it is possible that the "development history" of a certain knowledge element may be forgotten because memory is satisfied with the result of its constitution process - the core or goal of an action is maintained, whereas the way towards it is not kept in mind. On the other hand, the context of the meaning of a knowledge element may be lost while individual aspects of its interpretation are maintained.

When looking for the term "oblivion" in Schütz's work, it is striking that in the early publications, the term hardly appears at all. Then it appears in individual sections in the book on the Structures of the Lifeworld, which Thomas Luckmann completed, to allow for assumptions concerning its use for a theoretical concept. It is furthermore remarkable that - insofar as it is not used as a phrase in the sense of "we should not forget" - sometimes it is put into quotation marks. Such a reference to "inauthentic" language demonstrates that concerning this term the authors were aware of different levels of meaning.

Perhaps, Schütz and Luckmann always put the term "oblivion" into quotation marks when they point out a failure when recalling "actually" available knowledge. ${ }^{79}$ However, the word usage also shows two levels: on the one hand, it is about an actor forgetting to execute a once drafted action. "In everyday language", this failure is equated with "oblivion" if the relevancies or preferences

79 Here Heidegger's somewhat awkward term "ready-to-hand", which is used by Schütz himself, is helpful insofar as it addresses a kind of knowledge which is concrete and thus "to hand" - an objectified, objectivised kind of knowledge. 
for action have changed. After all, the focus is thus on giving reasons for rationalising a certain behaviour. ${ }^{80}$

On the other hand - and this is more important in terms of a theory of oblivion - it is stated that the inherent structure of knowledge contents shows different aspects for which the opportunity of oblivion is more or less likely. Sometimes somewhat vaguely, Schütz and Luckmann distinguish between a core and a periphery of knowledge elements. Whereas the core is maintained for a longer time, peripheral aspects are sooner forgotten. ${ }^{81}$ Here, oblivion is defined in the context of restorable knowledge. It is about "knowledge which has either been lost or hidden by other knowledge" (Schütz \& Luckmann, 1973, p. 176). The loss of knowledge may be due to "that certain aspects of an element of knowledge have been 'forgotten', although its 'kernel' remains" (Schütz \& Luckmann, 1973, p. 176). For example, one tends to forget about the complicated reflection processes while creating an experience - the modification of appropriate schemes - and only to remember the result of the process. In other words: not only the many perception contents of a current experience are forgotten, which after all are consolidated by one experience, but also the whole "story" of changing each respective scheme in the course of a learning process. When seeing a bonsai for the first time, one wonders and checks if this is "really" a tree. For all other experiences with bonsais, however, then the scheme of our experiences with trees has been extended by the impression of the small yet old tree. Usually, we forget about our astonishment, like about the stimulation to not make any attribution to the scheme of "tree".

In concrete terms, Schütz and Luckmann discuss the oblivion concept in constituting the social stock of knowledge. If - in the course of social change the attribution contexts between knowledge elements are interrupted, entire fields of knowledge may fall into oblivion. In this context, they indeed speak of the fact that "[t]hese then sink into oblivion" (Schütz \& Luckmann, 1973, p. 297). Schütz and Luckmann connect the possibility to recover such lost

8o Such a figure is found e. g. in case of not consulting a book on mushrooms after having collected mushrooms, to which Schütz and Luckmann (1973, p. 198) refer in the context of classifying the stock of knowledge for the value of the indications it provides. However, the desire to check once again something one is somewhat familiar with may disappear again if other things become more important - then one forgets what one intended to do in the first place. The situation is similar in the case of the also addressed use in the context of adjustment oblivion: one is so much captured by a situation that one forgets to adjust to the new situation (see Schütz \& Luckmann, 1973, p. 236) - thus, absentmindedness has, in the wider sense, something to do with "oblivion".

81 It is remarkable in this context that it seems as if only forgettability constitutes the distinction of core and periphery. Thus, there are aspects which are more likely to be forgotten and others which are less likely to be forgotten. 
knowledge to fix it in writing. By reaching back to sources, it is possible to reconstruct knowledge that has become irrelevant - in oral societies, however, such a kind of oblivion results in an irretrievable loss. Some of these explicit references to an oblivion concept correspond with the frequently implied indications one may refer to for suggesting an oblivion theory in Schütz and Luckmann. Indeed, Schütz's detailed analysis of the sense of action, the experiential context and the social stock of knowledge provides several stimulations and connection points for a theory of social oblivion.

However, apart from sometimes explicitly mentioning, as just discussed, the topic of oblivion, Schütz offers quite some implicit aspects of discussing the giving up on or the loss or decay of knowledge. Among these, there are the questions about the selectivities of a) the sense of action, b) the social stock of knowledge, and c) the relevance structures.

If both the subjective meaning any draft for action is based on and the meaning attributed to any observable behaviour - that is, when attempting to understand or interpret - is communicated by experience, it seems evident that precisely the process of attributing meaning is highly selective. This is connected to perception being always selective - a problem that will be discussed subsequently. However, Schütz initially answers this question differently. By stating that the consciousness spontaneously pays attention to everything which may be perceived around it (attention á la vie), he reaches back to a motif in Bergson. This attention is attracted by reality par excellence - reality as it must be experienced immediately. As the consciousness cannot split, its attention at a given moment always inevitably refers to just one part of reality; however, apart from the reality of everyday life, it may also be attracted by other realities; but the reality of everyday life has top priority. ${ }^{82}$ So then, experiencing each respective reality "automatically" - by way of addressing schemes - comes with creating an experience as a modification of the concerned schemes.

This idea may also be checked for oblivion, as both the turning towards currently addressable fields of reality and the recalling of suitable schemes always require more or less intended selection. Such a way of ignoring other possibilities of immediate experience as well as of the reflective genesis of experience, as it comes along with turning towards anything, will then result in the process of oblivion. Those schemes as not being addressed are not further developed, and recalling them is no longer part of the routine. Lack of concentration, distraction and weak retentiveness can be explained this way: if the view of the lively consciousness is preferably on everyday events, it becomes difficult to

82 Here Schütz (1972) adopts William James's concept of paramount reality. 
focus one's concentration - such as on the lecturer giving a lecture. ${ }^{83}$ Looking out of the window at the construction site nearby recalls different schemes than those of the reality of the academic genesis of knowledge. And indeed, the latter's schemes are no longer addressed and modified the moment one is distracted. Furthermore, it takes some effort to turn away again from the reality of everyday life and back to academic teaching or to "take up the thread" again. However, as the schemes have only sporadically or insufficiently been "connected" to each other or developed, it requires more effort to catch up with what has been missed. Otherwise, what could not be wholly integrated into the subjective experiential context will be forgotten again due to not being frequently recalled.

If this psychologically-sounding theory of the subjective experiential context is applied to the field of constantly interacting subjects, we will arrive at the construction of a supra-individual or social experiential context with Schütz. As also this experiential context has historically "grown", it is called the social stock of knowledge. Apart from each respective experience, it serves as the socialisation, process-communicated, basis of experience for the individual consciousness - each depending on a specific society or culture. ${ }^{84}$ The structural principles of the development and continuation of social knowledge are roughly like those of the individual. This means that contents may fall into oblivion also there. Schütz and Luckmann put this into words by reaching back to the German term "Nichtwissen" (negative knowledge):

Negative knowledge as hidden knowledge arises out of formerly positive determinations, which have been annulled and replaced by new positive determinations and hidden, so that they are no longer given in the familiar horizon of the element of knowledge. (Schütz \& Luckmann, 1973, p. 176).

Here, oblivion is described as a process coming along with any modification of the social stock of knowledge, only that Schütz and Luckmann have it in other words. Remarkably, Schütz equates negative knowledge with knowingno-longer. Obviously, he is not interested in other kinds of social negative

83 That ancient mathematician Archimedes of Syrcuse ignored such an attention á la vie perhaps cost him his life, at least according to the anecdote. All he had to say to the enemy warrior who caught him when he was making his calculations was "Do not disturb my circles!", subsequent to which he was slain (see Stein, 1993, p. 3).

84 However, equating the thus developed social stock of knowledge with social memory would suggest a space metaphor which understands memory to be a material store. But not the storing of knowledge is in the fore in case of the stock of knowledge. It serves for directing the attention of the attentive consciousness according to its specific having become. 
knowledge, such as well-known negative knowledge in the sense of not-yetknowing or unknown negative knowledge. ${ }^{85}$

Being a social memory, the social stock of knowledge is not only a store providing schemes - or types - to solve everyday life problems. It is also a selfcontinuing, that is changing over time, selection principle which decides to which memory image or scheme a consciousness reaches back. It has already been pointed out that in this context, Schütz introduces the concept of relevance. As an element in charge of controlling the subjective stock of knowledge, relevance structures determine the acquisition of knowledge - and thus the process of memory as connecting current perception contents with the schemes of the subjective stock of knowledge. The three kinds of (ca) thematic relevance, of (cb) interpretative relevance, and of (cc) motivational relevance must be distinguished. ${ }^{86}$

(ca) Thematic relevance is further structured into imposed or enforced, chosen, and hypothetical relevance. The former relevance consists of a previously unfamiliar subject of perception being forced into a familiar topical core. In this context, the crucial question is why, in a familiar situation, one is interested in certain aspects and not in others. This selectivity is due to the previously habituated perception habits. The second variant of thematic relevance is voluntary attention. In particular, when preparing for allegedly unfamiliar situations, with the help of expectations, one reaches back to classified knowledge. Thus, the memory prepares for perceiving a situation by pre-structuring the necessary definition of the situation by way of existing classifications. By the third sub-group, that of hypothetical relevance, Schütz describes how perception is structured by action routines which are imperative in certain typical situations. The goal is then to transform hypothetical relevance into valid relevance. An unknown sound in the house will be a reason for checking out by what is has been caused, in the course of which different consequences will be considered. Finding out about the cause determines the frame of defining the situation and transforms an unknown situation into a situation where the memory provides schemes and scripts.

(cb) In contrast to thematic relevance, in the case of interpretative relevance, the interpretational context is already given. This type of relevance can be tackled from two sides: certain aspects of a perceived object or certain elements of the stock of knowledge are used for interpretation. Interpretative

85 On this see the studies on the research of negative knowledge (in particular Wehling, 2006).

86 On this see Alfred Schütz (1971) as well as, providing the basis of this overview, Alfred Schütz and Thomas Luckmann (1973, pp. 224-229). 
relevance is a function of the biographically imprinted subjective stock of knowledge. At the same time, the environment determines which knowledge elements are activated.

(cc) Motivational relevance is connected to reasons for action. On the one hand, a perception may be characterised by an action goal the situation is based on - and which is pre-remembered. Such ways of perception are culturally shaped, as social groups have institutionalised certain ways of considering action courses and motivational contexts for typical, similar situations. Here any empirical assessment is complex, as the motivations must be expected to be meaningfully transformed as soon as the action has been completed: "What is 'teleologically' relevant when seen from the beginning, is presented from the end as 'causally' relevant" (Schütz \& Luckmann, 1973, p. 214). However, relevance may as well be biography-induced if a situation seems to be freely shapeable in view of the future but is limited in view of the past. However, we must not understand biographic influence as a specific knowledge element, but it is a syndrome of different elements stimulated by a subject of perception.

The different relevance structures cannot be considered in isolation from each other. Together yet to different degrees, they cause the constitution of experience by activating certain aspects of the existing stock of knowledge in the given situation. Closely connected to this, relevance structures thus change the stock of knowledge in specific ways. This change, however, can only be interpreted in retrospect and thus only by the resulting relevancies; devalued relevancies fall into oblivion.

The application of the result of eplication as an element of knowledge in the mastery of an actually present, typically similar situation is determined only by those relevance structures which concern the end result of the explication, its monothetic meaning, and not by alle the structures which are more or less at the basis of the polythetic process of explication. (Schütz \& Luckmann, 1973, p. 228)

From the many connections to issues of memory, of remembrance, as well as to oblivion to be found in Alfred Schütz's work, we may derive a genuinely sociologically theory of memory. Starting from the egologic perspective of the experiencing I, Schütz demonstrates that any social action is basically tied to memory. Accordingly, any action as drafted behaviour is based on a pre-memory and is thus characterised by the individual stock of knowledge. Analogously to the stock of knowledge of the individual, which develops from memory-related schemes, the social stock of knowledge constitutes itself as the total of objectivised knowledge all individuals of a group are provided with. However, the social stock of knowledge as such cannot be equated with 
social memory, as according to Schütz's concept in the case of memory, there always happens a comparison of similarities, in the course of which the new is compared to the known, which way how perception contents and stocks of knowledge are related to each other are determined.

The question of which (classified) memory images or schemes of experience becomes topical in specific situations, thus of how the situation-specific selection of stocks of knowledge is organised, is answered with the help of the concept of relevance structures. There Schütz provides a differentiated theory of different kinds of partly individual-biographic and partly culturally communicated selection mechanisms which is helpful when it comes to an understanding of situative perception according to its social conditionality on the one hand and to action consequences which are typically derived from it on the other. Therefore, $n$ the shadow of this constructive process of developing social knowledge, at the levels of the subject, the collective, and the social order structure, there happens a structurally similar process of permanent oblivion. With each updating of the individual or social schemes happening within the frame of defining situations resulting from comparing a status quo to new impressions, everything currently does not seem relevant is step by step deleted from the current relevancies. Thus, oblivion in the sense of Schütz develops in the course of the process of the classifying constitution of schemes, of attributing perception experiences to existing schemes as well as of their permanent adjustment to a perception which at the same time is selective according to the socially communicated relevance structure.

Connecting to the oblivion motifs worked out in Schütz, Gerd Sebald distinguishes five kinds of oblivion that refer to the changing schemes - which are also classified with intersubjective communication (Sebald, 2011). Three variants of oblivion - the intersubjective one, the generalising one, and the structural one - derived from what has been said so far. Then, perspectival oblivion is what he calls the fact that attention can always use only one currently addressed relevance system. Everything beyond this structure of attributing meaning is either ignored right from the beginning or forgotten at once. Finally, the fifth kind of oblivion is transmarginal oblivion. This variant, oriented at Schütz's concept of the transmarginal consciousness experience, aims at the problem of what can be reproduced and anticipated, in the context of which the question about the reference to once existing - thus forgotten knowledge is raised. Also, this is about the phenomenon of experiences being eclipsed in the course of transforming them into schemes, in the context of which it is assumed that there is knowledge of what-has-been-known-in-thepast. This suggests the assumption that configurations of schemes are provided with a time index allowing for the insight that "it" must have been known 
earlier - in a different or better way. Access to this kind of oblivion is possible most of all when finding a trace and thus starting pursuing it.

\subsubsection{Oblivion by Way of Incorporation}

Incorporated, implicit or practical knowledge is characterised by having become habitual or a matter of course. Any declarative reflection, any intention or detailed action plan is no longer necessary to trigger or give reason to applying and using such a kind of knowledge. The orientation of sociological research at so-called practice theories has produced the insight that vast parts of social and societal order consist of implicit arrangements. Both Alfred Schütz and Peter L. Berger, as well as Thomas Luckmann frequently pointed out the great significance of this implicit, habitual, matter of course and thus unquestioned everyday knowledge. ${ }^{87}$

Working into the social world is based on assumptions and processes whose acquisition and occurrence have been forgotten. Sometimes the behaviour resulting from such a habitual knowledge has been practised and incorporated into the body so that we may speak of incorporation whose consequences are obvious for phenomenologically trained observers but not for those individuals or groups as being concerned by this behaviour. A crucial motivation for oblivion by way of practising and imprinting is to relieve the systems of information processing and the reduction of decision-related selection requirements. In other words, one can forget any reflection on the meaning of one's behaviour and explanations for its original meaning. As a result of giving up on reflecting, due to routine, the fact that movements and gestures and body features may be meaningfully questioned. By systematically ignoring possibilities, this simplifies everyday practices, as simply there seems to exist just one possibility to act, and that is the chosen one. This way, any change of habitual behaviour is prevented. Only by relieving oneself of the everyday-practical

$87 \quad$ Accordingly, Schütz - as already stated - does not only recognize the problematic access of reflective knowledge at motivations for action coming from the past; he also assumes a wide field of routine and habitual knowledge which has become a matter of course. This insight is already to be found in The phenomenology of the social world, when it is about Weber's type of "traditional action" which is presented as incorporated, habitual, standardised action or behavior (Schütz, 1967, p. 197). The concept of habitual knowledge is discussed in detail in The Structures of the Lifeworld (Schütz \& Luckmann, 1973). Berger and Luckmann (1967) include this into their fundamental principles of a sociology of everyday knowledge. Their concept of institution, which is based on habitualisation and objectivation and furthermore connects to the concept of collective consciousness according to Durkheim, is nothing else than a kind of social and societal order which is not discussed anymore and, in its given form, is taken for granted by the respective social group. 
pressure to act, routine as an inflexible order can be put up for renegotiation. One way of achieving this is a kind of awareness that remembers the inhibition of oblivion - although even this is necessarily always a permanent construction of memory.

However, the first theoretical addressee for such considerations in sociology is not social phenomenology but the theory of practice connecting to Pierre Bourdieu. There, motifs of implicit action and behaviour are unfolded, similar to those mentioned above. For example, habitus is nothing else than an incorporated, structuring structure in the sense of the memory concept. Then it seems to be obvious to raise the question of which oblivion processes are at work when sometimes extendedly practised knowledge has finally been incorporated to such a degree that one does not at all reflectively "remember" how it was acquired. The entire realm of everyday routines is based on comprehensively, subjectively forgetting about how it was realised. In his analysis of oblivion in Bourdieu's works, Peter Wehling points out that not the knowledge contents have been forgotten but the situations of acquiring this knowledge they have been deleted from the memory of events. It is remarkable in this context that the individuals have forgotten not only this. They have also forgotten that they have forgotten the learning situation as such. While connecting to a corresponding insight by Hahn, Wehling calls this the oblivion of oblivion (Wehling, 2011b).

Alois Hahn illustrates this motif by the example of forgetting about language acquisition. By "learning" he understands the imprinting of culturally relevant distinctions. He understands remembrance - in contrast to the here unfolded declarative-reflective interpretation - as being possible both explicitly and implicitly. Accordingly, any implicit, routine or automatized reaching back to learned knowledge is called "operative remembrance". Accordingly, Hahn says, unlearning as a special kind of oblivion happens if such an automatic way of reaching back does no longer work.

Of course, in the context of language and being able to speak, this does not only hold for the operative memory of phonetic differences but just the same for as spontaneously mastering grammar and vocabulary. [...] Language acquisition starts with oblivion: The baby's babbling and so called deaf-dumbness (understanding or speaking) prove that there is neither a lack of motoric capabilities nor of acoustic impressions, and yet most of the sounds are suddenly lost again. Thus, learning how to speak is a 'selection of sounds'. (Hahn, 2007, pp. 41-42)

Which of the baby's original sounds are remembered and which are forgotten is different according to culture. Thus, it is "operatively forgetting other possibilities", which might be used in other cultures (Hahn, 2007, p. 42). Apart 
from alternative possibilities to articulate also the once-happening selection is forgotten. ${ }^{88}$ Only the distinction itself is maintained as a knowledge structure.

Thus, regarding oblivion through incorporation, we may state that by practising, exercising, and imprinting, there happens an acquisition of knowledge that is then unquestioned, practically available and indeed applied. Oblivion is a specific feature of this kind of knowledge, as usually the entire acquisition process is not remembered - then one is simply "capable of".

In Bourdieu, however, practising such behaviour does not happen "purposefully" but in line with the practice of the social field within which each respective actor acts. Both in social reality and the reality of individual fields, the individuals "notice" how to behave successfully. ${ }^{89}$ Sometimes the groupspecific view at success is imprinted into gestures, attitudes, taste and other ways of social expression and social distinction without further being explicitly reflected on. That habitus serves for stabilising the respectively predominant power relations is well-known. By way of a memory-theoretical reading, however, this understanding can now be extended: much more important than remembrance, that is habitual behaviour, is oblivion as well as corresponding oblivion of oblivion; in this context, oblivion appears as an instrument of domination which can be a subject of discussion.

\subsubsection{Social Theory}

If, on the one hand, we may inform ourselves about how oblivion happens in the context of individual consciousness or collectives, given identifying subjects of oblivion (oblivion subjects), we may ask what is typically forgotten. Concerning this aspect, however, the focus is not on what has objectively been forgotten but on that what, in the form of perceiving traces, is recognized as having been forgotten. This is based on the assumption that what a consciousness (system) is capable of identifying as having been forgotten is only a small part of what has actually been forgotten. ${ }^{90}$ In other words: the now following considerations will be about socially reflecting on oblivion which, however, does not derive from noticing that constantly something is being forgotten. Rather, it is about the efforts made by individuals and collectives to keep forgotten things present or to re-update them. The concern about the danger of

\footnotetext{
88 Very similar considerations are also to be found in Paul Connerton (1989, p. 101).

89 For an overview see Pierre Bourdieu and Loïc Wacquant (1992).

9o If this argument is applied to the memory research of cultural studies, one encounters the well-known contradiction between history and (collective) memory. Collective memory covers the social reference frames triggering individual remembrance as a precondition for meaningful action. History, on the other hand, deals with a sources-guided (re-)construction of meaning by help of past events and contexts.
} 
oblivion must be equated with the concern about leaving a vital experience out of consideration when drafting future actions and, against better knowledge, about missing adjustment opportunities. And these adjustment aspirations do not only conclude from the experience of the "burnt child" but also include documenting the family history as proof of status, the keeping of documents on far-reaching company decisions or the writing of national histories to give historical reasons for territorial claims on the one hand and as a contribution to a collective identity on the other. Thus, archiving is the first protection against the fear of forgetting knowledge that has been recognized as relevant, and the retrospective construction of historical contexts is the second protection against this fear. To have it somewhat more extendedly, we might as well speak of more or less explicitly controlling the maintenance of the structure, on the "flipside" of which there always happen processes of oblivion. Parsons's sub-system of latent pattern maintenance, for example, describes a function of social memory which, as a side effect, constantly produces oblivion - everywhere where no deprivation is experienced. ${ }^{91}$

If we understand the topical field of social theory as a perspective dealing with the analysis of social order, theoretical approaches in the tradition of functionalism and structuralism seem to be the first choice. In the following, approaches shall be discussed which deal with oblivion. In this context, the approaches by Maurice Halbwachs (1), Michel Foucault (2), and Niklas Luhmann (3) seem to be promising. All in all, it is about the question of how far oblivion is constitutive for societies against the background of an ordertheoretical or structural-theoretical perspective.

No explicitly formulated theory of oblivion is to be found in the works by Halbwachs. Nevertheless, frequently he contrasts frame-guided memory to oblivion - and he seems to prefer a certain concept of oblivion. Oblivion is first a process of a loss of knowledge given the lack of each specific social reference frame. This explains the difference between perfect memory - imaginable only as an ideal - and the trace as stimulation for memory provided to retrospective thought by the individual memory. According to a metaphor by Halbwachs, such a trace is like a crystal core which is put into a saline solution and which then, in a way in a context of suitable material, may grow again (Halbwachs, 1980, p. 25).

Total oblivion means that no trace is to be found. In this context, Halbwachs refers to being reminded of a common experience of which does not remember the slightest trace - one knows that one must have been there, but by no

91 Some elaborations on the issue of social memories in Parsons are to be found in Marco Schmitt (2009). 
stretch of one's imagination, one remembers the situation. However, this phenomenon is not explained in terms of neuro-psychology but of sociology: the capability to remember situations is connected to the opportunity to renew these memories with certain groups sharing the same memories. According to Halbwachs, oblivion as a natural process always happens when there is no reason to remember - and this may, on the one hand, be connected to lacking emotional ties to these experiences and, on the other hand, to the social context of memory having been lost. Leaving a group for good, for example, results in forgetting about the experiences connected to it.

[T]o the extend to that the dead retreat into the past, this is not because the material measure of time that separatesthem from us lengthens; it is because nothing remains of the group in which they passed their lives, and which needed to name them, that their names slowely become obliterated.The only ancestors transmitted and retained are those whose memory has become the object of a cult by men who remain at least fictitiously in contact with them. (Halbwachs, 1992, p. 73$)^{92}$

Oblivion is triggered by the disappearing of reference frames as well as the frames changing or being changed over time. ${ }^{93}$

Another example of such a frame is language, which changes slowly and may disappear with a culture or group. In this context, words are not just attributions but contexts of attributions accompanied by manifold memories. Not the past is stored by the memory; "it is language, and the whole system of social conventions attached to it, that allows us at every moment to reconstruct our past" (Halbwachs, 1992, p. 173).

At first sight, the historian and philosopher Michel Foucault, who is also of significance for the sociological debate, does not look like an authority of a social-scientific theory of oblivion. ${ }^{94}$ He is interested - we have already referred to this - in an "archaeological" and "genealogical" reconstruction of social discourses. Discourses are "bearers of the positivity of statements" (Keller, 2011, p. 125) - thus, associating them with concepts of social memory or

92 This may be completed by the statement that the memory of a society reaches as far as the memories of the groups it collects. In this context, oblivion does not happen intentionally but by individual groups - as the bearers and initiators of each specific memory simply disappearing (see Halbwachs, 1980).

93 On oblivion as result of leaving and changing social reference frames see also Jan Assmann (1991).

94 For Foucault, "oblivion" is no theoretical element, although - like many other theoreticians - he frequenty points out to this phenomenon. In his reconstruction of the oblivion issue in Foucault Reiner Keller (2011) states that, apart from scattered indications of oblivion, strong implicit references can be identified. 
remembrance is more evident than connecting them with issues of oblivion. However, oblivion comes into focus when becoming aware of the selections he discusses in his theory of discussed selections. One crucial motif of Foucault's discourse analysis is that discourses, as orders of how to speak about something, always depend on the social power relations of a given time. Suppose the social construction of knowledge and truth depends on power and power mechanisms, with Reiner Keller. In that case, we may conclude the assumption that given the organising "running" of discourses, selections are made firstly in the form of bans, taboos or etiquette, secondly concerning the distinction between madness and reason, and thirdly concerning the distinction between true and false. ${ }^{95}$

Another way of selectively structuring discourses are procedures with the help of which discourses become self-controlling: principles of classification, organisation and distribution. In other words: it is about how the "language" used for the discourse organises, consolidates or, in the form of comments, connects to something existing. Discourse-inherent mechanisms of sanctioning e. g. in the context of the sciences, Foucault refers to disciplines that decide who is allowed to comment on what and when - take care that the rules developed in the course of the discourse are kept. Such a limitation for possible contributions is mainly realised by way of specific qualification guidelines.

These selections are the preconditions for oblivion which then happens by the constant becoming and vanishing of discourse formations. The psychological idea of the cognitive scheme is transferred to the socio-historical idea of a social topic for which everything becomes irrelevant which is not updated. The internal control of the discourses appears as preparation for such a kind of oblivion, as not least there it is determined who is heard or seen and who not. Thus, a damning review is less problematic for an author than his/her contribution being ignored, as in the latter case, the discourse does not appreciate the trace he/she has pointed out but makes it fall into oblivion by ignoring him/her.

For the time being, the theory of social systems may be considered the only sociological approach for which the concept of oblivion has an explicitly theory-architectural value: there; oblivion is taken as the crucial aspect of the systems-theoretical concept of memory. In the following, some aspects of the systems-theoretical concept of social oblivion shall be taken into consideration more closely (Dimbath, 2011a).

Social systems prefer certain condensates of meaning and this way become capable of observing the identical and the similar in their environments. This 
way, it is possible to identify information as "already known". If it can be meaningfully connected to an already existing condensate of meaning, Luhmann uses the concept of scheme or script - the processing of information has been completed, and the communication process is forgotten. However, if the consistency assessment produces an incomplete congruence, the informationprocessing structure, which is understood to be a scheme, must adopt the new impression and adjust accordingly. Thus, any communication that adopts new information, resulting in a change of meaning condensates, supports the system's inner differentiation. However, a piece of information that cannot at all be related to already existing structures is "too new" and can thus not be "processed": the system "sees" only what it can "see". In this context, memory appears as a highly selective exceptional operation. It is a reflection process aiming towards the "inside".

Thus, memory in the sense of social systems theory is no storage place but a mechanism performing a consistent comparison with the systemic communication structure "condensed" by the scheme. This way, the system produces an always updated idea of its own time and past - sometimes, it even adds its own time index to certain schemes to provide orientation within "historically relevant" contexts. In the further course of the system's memory-induced operations also the memory itself changes. Thus, it is no static "switch" but has a channelling function that is frequently adjusted to current conditions. If remembrance happens in exceptional cases, this is a suppression of "automatically" giving up on memory which Luhmann frequently calls oblivion. "The main function of memory, therefore, lies in forgetting, in preventing the system from blocking itself by concealing the results of earlier observations" (Luhmann, 2012, p. 349).

What is remarkable with such attribution of function is that here oblivion is taken out of the realm of passive-unwanted situations and is presented as an active task. This is based on the idea that the system is constantly confronted with an overwhelming mass of information to be processed, which cannot be assessed for suitability for autopoietic follow-up communications, then sometimes be transformed into the system's history. Memory is helpful in this context because it rejects all information being irrelevant in this sense, which keeps information-processing capacities available. It is the fate of the "rejected" information to fall into oblivion. ${ }^{96}$

96 However, this raises the question of in how far information which is not stored to be further processed may already be counted among the "intellectual" property of the system. After all, however, it is inherent communication which has resulted from an impression which is counted as being environmental. Even if the system is not yet "aware" of it, still it 
In the further course of information-processing, there frequently are renewed consistency assessments using the respectively updated structures of information-processing: this means a comparison with existing schemes. The information which can be made congruent with existing structures or can be "subsumed" under them is not further processed and forgotten.

The two variants of oblivion in social systems presented thus far must be considered selections - similar to the selective perception by individual consciousness. In everyday life, we might speak of "overlooking" and "ignoring" in one case and of "recognizing" and "attributing" in the other. Luhmann calls this making capacities of information-processing available because the system can decide very soon if it must deal more closely with information from its environment and, if necessary, "adjust". ${ }^{7}$

Another function of oblivion - also discussed in systems theory - is preventing memory. Memory must be an exception because otherwise, the system would face the task of mostly dealing with its own "memory information". A system exclusively dealing with its past would neglect current impressions from its environment and would thus no longer be capable of performing vital adjustments. This is what Luhmann means when speaking of the system blocking itself. In Luhmann, we do not find any information on how this suppression of the "inner voice" happens exactly; but we may speak of oblivion insofar as it ignores, does-not-take-into-consideration does not connect existing information.

The third variant of oblivion refers to the structure of information processing itself. The memory decides which structural field or which scheme is used for consistency assessment. Meaning condensates that have not been used for some time have fewer opportunities to be reactivated. Thus, oblivion happens by today both "attention" and the "attribution of meaning" looking different from some time ago and because today it cannot be understood why then a specific follow-up communication could be successful.

The systems-theoretical concept of oblivion shows different variants of oblivion which are also to be found in the "cultural history" of the concept of oblivion. Probably Luhmann did not have time enough to work out the theoretical element of the social memory, with oblivion as its main function, in more detail, so that this aspect of his late work stays incomplete. ${ }^{98}$

is already there. Thus seen, the system forgets something of which it does not yet "know" that it exists, as no production of meaning has been started.

97 The theoretical terminology of social phenomenology deals with this as "relevance".

98 In Luhmann's works there are comparably many passages referring explicitly to oblivion or memory. The most elaborated concept is presented Theoryof Society (Luhmann, 2012); 
In short: object-related and culturally triggered oblivion happens along with topical selection decisions. This not only holds for sub-systems but also for the categories of discourse or the province of meaning or world of meaning. Excluded is what does not belong; in the context of religion, this is everything non-religious; in the context of legal issues, it is everything not belonging to the legal sphere and so on.

On the one hand, the systems-theoretical view at knowledge, memory and oblivion is, in a knowledge-sociologically reflected way, provided with a degree of sensitivity to implicit assumptions concerning the kind of applied knowledge. On the other hand, this position underlines the fundamental doubt that oblivion could, in different contexts, always happen according to the same principles. However, it is an open question if this scepticism, based on the assumption of differentiation, does not go too far. The assumption of contextdependent kinds and practices of oblivion, which are thus basically different from each other, protects from an all too universalist approach; however, if oblivion processes must be considered so much disparate requires further assessment be done here.

\subsubsection{The Diagnosis of the Present Time and Social Change}

After having collected sociological findings on oblivion in the context of theories of sociality on the one hand and social order on the other, sociological theories dealing with the description and explanation of social change remain. ${ }^{99}$ Sociology, a "product" of modernity, shows a simple yet crucial motif of reflecting on history. Usually, it construes social change between two epochs: the "classical" distinction is between tradition and modernity, whereas since several years ago, it distinguishes between post-, late, or advanced modernity or between first and second modernity. Both sociologists and historians assume that memory or oblivion might have changed their nature in the course of the transition between these epochs. In this context, modernity is usually considered the age of oblivion. In the following, four of these positions - oblivion becoming stronger due to the change of storage media (1), oblivion becoming stronger due to acceleration (2), oblivion as a result of becoming insignificant (3), and discourse-communicated oblivion as a result of power effects (4) - will be shortly presented, in the context of which any distinction according to disciplines is not considered to be relevant for structuring.

a critical overview of the many hints at memory- or oblivion-theoretically relevant issues is provided by Oliver Dimbath (2011a).

99 Here it is about contributions which are exclusively sociological - both the humanities and cultural studies provide a wide range of interpretations of modernity as the age of oblivion. Some of these aspects have already been pointed out to in the first part of the here presented study. 
Elena Esposito offers an evolutionary approach to the development of social memory. Although reconstructing periods of memory practices undergoing epochal change to different degrees, she focuses on the transition from industrial modernity to the IT age. Her cultural-historical analysis of memory starts with oral, illiterate cultures dependent on remembering through constant repetition: what is considered culturally relevant is frequently presented in different ways and thus passed on from generation to generation. In those times, the distinction between remembrance and oblivion is a fluent contradiction. In early literate cultures, textualisation is understood as fixation, which does not produce any new insights in contrast to living memory. Information one believed not to remember was fixed with the help of documents. With the establishment of functional differentiation, culture is equated with memory. Now priority moves from remembering to forgetting - with printing, no longer the aspect of producing memory aids is in the fore, but relieving the memory: stored which need not be kept in mind. Knowledge is outsourced into books, like "relieving the consciousness by liberating the presence, i. e. making oblivion easier" (Esposito, 2002, p. 240). The mass spread of information technologies makes Esposito assume that a new kind of memory, a kind of network memory, might establish. However, telematic memory does not show any fundamentally new memory practices resulting from the change of differentiating. It is only that the computer joins the archive and the books as a faster and, most of all, globally networked medium. The change of memory happens as a reaction to an ever more accelerating logic of intensification. Thus, we may say that information and communications technology radicalises the archive material by storing certain contents while deleting others. Additionally, increasingly reaching back to stored data happens automatically or by way of algorithm-controlled access. We may thus say that the machine produces answers to questions that up to then had been non-existent. ${ }^{100}$ Parallel to such radicalisation of the store and its administration, there develops a new technique of structuring oblivion. Those information contents as not being stored as data and appropriately catalogued are forgotten. The electronic archive always refers only to a certain kind of information, and given the exuberant amount of this information and the thus connected utilisation opportunities, other information about the world increasingly appears as being irrelevant.

Very much like Halbwachs, Paul Connerton orients the social memory at the dimensions of space and time (Connerton, 2009). He is interested in pointing out the many association and remembrance opportunities connected to streets, squares, and memorial sites. In his answer to how modernity forgets, he attests a kind of systematic oblivion to Western industrial modernity, which,

100 On this see Esposito's (2002, pp. 291-303) considerations on computers and telematics. 
he says, is most of all due to acceleration. Modernity has produced a culture of oblivion in the context of which much of what has up to now been maintained is quite purposefully left to rot. Examples of this are the politically expedient renaming of streets and squares, the rapid growth of cities, at whose fringes there develops something new in the shortest period without any claim to "historicity", or the change of perceiving space as a result of accelerated means of transportation. The stroller experiences the space he/she crosses on foot differently from that of the car driver.

Eviatar Zerubavel unfolds a narration-theoretical interpretation of the memory of modernity in his considerations on the cyclic nature of grouprelated self-descriptions or narrations (Zerubavel, 2003). This is based on the insight that it is a fundamental feature of the human memory to embed unstructured series of events conceptually into allegedly coherent narrations. The structure of such narrations is subject to clearly identifiable patterns, such as the narration of progress or the narration of decline. One of the narration figures reconstructed by Zerubavel is characterised by distinguishing the mountain from the abyss. There, the distinction is between historically more significant and less significant events. By the different intensities of labelling, it is possible to understand why some events are perceived as widely visible, like peaks, and are thus kept in mind, whereas others sink into the abyss of being narratively insignificant. This way, collectively binding presentations of history produce "dark ages", which, as periods that are said to be poor of events, fall into oblivion.

"History takes up the shape of a topographic map where memory-relevant events of the past and those to be forgotten are marked like mnemonic hills and valleys" (Zerubavel, 2003, p. 27). A variety of such narration figures identified by Zerubavel can only develop in modern society in which dealing with the past is no longer authoritatively but collectively organised.

Analyses of the consequences of social change can also be made against the background of the discourse perspective unfolded by Foucault. In the context of a "discourse economy of oblivion", Reiner Keller identifies five changes: ${ }^{101}$ Due to increasingly focusing on the exploitability and utilisation of knowledge, he says, firstly, a trend towards an economised will to truth becomes apparent. According to this priority, everything which is not convincing in the light of evaluations and rankings may be forgotten. Against the background of increasing globalisation, Keller secondly states that anything regional and

101 Reiner Keller distinguishes six lines of change, of which one - the "transformation of the comment" (Keller, 2011, pp. 129-135) - can be referred to two others, which is why here only five processes are mentioned. 
local is pushed aside. Only that is considered relevant for being continued or restarted, which is recognized as global knowledge or knowledge which might be globalised. Thirdly, the attribution of relevance in discourse - as Esposito also states it - aims at digitalised knowledge and knowledge which can be digitalised. Everything which cannot be digitalised may fall into oblivion. Fourthly,, the disciplines of the sciences are subject to change which, may be described as de- and re-disciplining, says Keller. Against the background of the call for inter- or trans-disciplinarity, the "grown" disciplines are pushed to the back so far as attention and cognition are granted to new XY studies. Finally, Keller states a shift in attributing attention towards specific media formats. Due to increasingly preferring the visual, there develops a trend of avoiding less accessible information such as texts. This way, Keller says, also the non-visual and that what cannot be made visual is neglected and falls into oblivion, after all.

When discussing oblivion in sociology, a difference to cultural studies perspectives can be identified: with sociological approaches, we rarely find the idea of memory as a store from where data are lost, which could be equated with oblivion. Sociologists - particularly those feeling obliged to constructivist ideas - rarely adhere to the idea of knowledge stored elsewhere than in the psychic or social consciousness. For them, knowledge is an always present structural aspect that is imagined as a structure of schemes and scripts organised by memory. On the one hand, oblivion happens in the course of information processing through superimposition and decay. On the other hand, it happens in the course of the evolutionary change, though activation and deactivation - i. e., selection - of situative patterns of processing. Reaching back to consciousness structures is crucial in this context to allow for experiencing similarities. Any continuation and, at the same time, self-transformation of the structural context happen along path-dependencies. Oblivion becomes marginal, that what is not needed or that what is not functional.

However, there is still the chance of oblivion being initiated by taking away one object. In this case, oblivion has nothing to do with this object as such but rather with the loss of possible orientations for follow-up communication and remembrance, which may quickly happen due to the loss of such an object. At the same time, however, there is still the possibility - although it is dwindling over time - that this object, precisely because it is missing, gives reason to remember it if context-related traces or empty spaces are perceived. Thus, social oblivion cannot only be understood as structural determinism or automatism. It may also happen as a result of decisions to delete, destroy or clean.

Sometimes, preconditions for oblivion are discussed in sociology if memories are purposefully avoided by intended selection - sometimes by third 
parties or in the course of discourse or also in the social field. This produces the effect that the social knowledge structure or classification of social phenomena may further develop so that certain facts are purposefully forgotten. Although everyday consciousness is incapable of forgetting on purpose, this may indeed happen in the realm of the social. The "nature" of oblivion - and this is well comprehensible in connection to sociological theories - may be transformed into a "culture" of oblivion. Such processes are described most of all in the context of the analysis of creating and maintaining social order. However, against the background of discussing social or institutional change, it is assumed on all sides that oblivion has even become the fundamental driving force of restructuring processes since the transition to modernity, due to acceleration processes as well as the rapid growth of the amount of information to be processed in many realms of the lifeworld. Thus, modernity would be much more forgetful than any preceding age.

\subsection{Basic Traits of Social Oblivion}

When it comes to the phenomenon of oblivion, we are provided with several starting points: at first, the range of the concept in everyday language has been measured, and afterwards, ways in which the concept has been culturalhistorically interpreted have been assessed. Then, some dimensions of the concept were distinguished by reaching back to the root word. Finally, we started looking for explicit and implicit motifs of an oblivion theory in the context of sociological theories. If we assume the issue of oblivion to be connected to timeliness, memory and remembrance, it must be clarified how these concepts are interrelated. This has been done in the immediately preceding sections; hence, the preliminary work for developing a sociological concept of oblivion has been completed now. Furthermore, the collected elements will be organised, opening them up for sociology and combining them with the already delineated sociological concepts of time, memory and remembrance.

\subsubsection{Two Dimensions}

As it has already been pointed out several times, the loss of (intellectual) property may be described by different concepts. Three of these concepts are also common in the social sciences and the cultural studies debate. The loss of stored information, which is usually also counted as oblivion, should be more appropriately called deletion, disappearance or destruction. In contrast, the overlooking or neglecting of obviously existing information, which is sometimes even described as "deliberate" or "intended" oblivion, may be defined 
as ignoring. Although it does not seem to be reasonable to exclude both the variants of the material loss of information and of giving up on information from a sociological concept of oblivion - this would produce unnecessary misunderstandings - there is nevertheless a kind of oblivion which may be described as "pure" oblivion in particular regarding the far unfolded memorytheoretical aspects. In the following, oblivion will be distinguished in the wider (1) and the stricter $(2)$ sense.

By oblivion in the wider sense - or oblivion (I) - we mean those ways of losing or giving up on knowledge that do not concern the process of oblivion as such but are its preconditions. This is of importance particularly from a sociological point of view because the fact that knowledge, or better: information, is existing can always be stated only from an observer's point of view. Thus, it is assumed that there is information that may be considered relevant in a certain situation. Now, that content is not recalled can be because the nexus indicates it is no longer existent and that access is blocked or unwanted. In all three cases, however, it is not that the concerned information has already disappeared. Instead, inter-subjectively comprehensible or classified traces indicate its existence. Thus, the expectation that the concerned information exists can be based on objects.

Several times, it has been pointed out that a social memory cannot be understood as a store. However, in everyday language, it is sometimes difficult to not connect the destruction of archives to oblivion, for example. Still, there is one crucial difference. When the library of Alexandria ${ }^{102}$ or the Anna-Amalia Library in Weimar in 2004 was destroyed by fire, or when the Cologne City Archive collapsed in 2009, a significant number of valuable documents were destroyed or lost. Both their contents and those impressions which can only be gained from the material presence of an object run the danger of falling into oblivion.

From the perspective of a sociological concept of memory, however, archived knowledge is of no presence-constituting relevance as long as it is not used to identify, define, and assess current situations by actors or systems. Thus, even the stocks of any archive are forgotten until they are needed and recalled: even at a library, a work may fall into oblivion. ${ }^{103}$ Deleting a hard disc,

102 The fire of the library of Alexandria - also shortly mentioned by Harald Weinrich (2004, p. 19) - is a myth. There are several historical reconstructions of the disappearing of this archive, but also the assumption of its destruction by the Arabs in the 7 th century has probably proven to be wrong (Lewis, 1990).

103 See Aleida Assmann (2012) who reaches back to Friedrich Georg Jünger's (1957) concept of storage forgottenness (Verwahrensvergessenheit). On this, Dietmar Rieger says: "Quite close to the cemetery metaphor is the attic metaphor [...] by way of which the symbolism 
a library burning down, the burning of books, all these are just preconditions for but not the process of oblivion. Thus, on the one hand, oblivion appears as a consequence of an accident or disaster. On the other hand, such a condition may as well be initiated, so that - once again by limiting the potentials of social memory as it became apparent in the course of the burning of books by Chinese Emperor Qin Shihuangdi (Strähle, 2003) or in the Third Reich - it just achieves incomplete oblivion. Thus, any conscious deleting or destruction is tied to the social because the actor purposefully makes information disappear, hoping that in its existing shape, it will be no longer relevant or a subject of remembrance. Possibly the actor him-/herself will be the last witness and thus the trace indicating this disappearance. Who wants something to be forgotten does not necessarily need to forget him-/herself. This can only be imagined in the context of counting on one's forgetfulness, for example, by eliminating remembrance stimuli such as photographs, presents, souvenirs reminding of broken love. ${ }^{104}$ However, such intentional oblivion is difficult since one will easily fail because of the social frame of collective memory. ${ }^{105}$

Another variant of intentional oblivion, which may be grasped as a precondition for oblivion, can be well illustrated by the phrase "You can forget about it!". The meaning of this statement is to devalue the relevance of certain information for future use because it will be (or is said to be) useless. This recommendation aims at ignoring, overlooking or neglecting certain information. This is sociologically significant as it expresses the expectation that the concerned information might be recalled and recognised as relevant by others. However, the expected perception is influenced. Accordingly, somebody having a clear idea of how a particular goal might be achieved may tell his/ her partner which information, in his/her opinion - and his/her interest - is helpful and which may well be ignored. In this case, the oblivion demand aims to recommend "put something right out of mind". Indeed, this may also be

of the attic as a storing place is made fruitful: 'storing' yet also marginalisation, indeed the exclusion of what is not needed, has become obsolete, of the forgotten traditions or traditions which shall be forgotten of earlier generations on the one hand - freedom from taboos, valid norms and constraints, the return to values which are no longer contemporary - yet authentic - the view to the open, the trigger for not least poetic, imagined memory on the other" (Rieger, 2004, pp. 26-27).

104 On this see the film Eternal Sunshine of the Spotless Mind by Michel Gondry (2004) which is extremely illustrating for many aspects of sociological considerations on oblivion (Germ.: Vergiss mein nicht!).

105 A literary example of this is provided by Max Frisch's novel Stiller, whose main character, Anatol Ludwig Stiller, cannot escape his "past" when he returns to his home, despite having obviously been successful with individual oblivion (see Heinlein \& Dimbath, 2010, p. 276). 
one's own intention: the actor decides to resist an expected perception and ignore certain, possibly relevant information. When pretending to not wanting to know something, this implies that one does not want to be reminded of the horizons of the meaning of the concerned information. ${ }^{106}$ However, such an oblivion demand will be successful only if it is obeyed and the goal is achieved without further irritation. In other cases, one will be reminded of what has been neglected or overlooked.

In the case of deletion and ignorance, we may speak of oblivion in the wider sense. ${ }^{107}$ Given the here unfolded theory of social memory, however, it is not about the oblivion process but about creating the preconditions for later oblivion. Also, these variants are of significant sociological interest. ${ }^{108}$

Oblivion in the stricter sense (oblivion (II)) should exclusively be understood as the process of oblivion. First, however, it must be clarified how something like this is supposed to be possible in the social context. The previous considerations on social memory provide one approach. Only if it is clear under which circumstances we speak of stocks of knowledge, an analysis of the loss of knowledge is possible.

Social memory has been described as an aspect of order which produces a continuity of a before, a now and perhaps an after, thus producing the idea of time for the processing consciousness. The memory structure consists of networked schemes and scripts or socially constituted types or (interpretation) patterns from which the memory - and this is its function - selects relevant structural patterns for the organisation of current behaviour and a pending meaning-related definition of a situation. Thus, oblivion involves selecting one possibility from different reference possibilities for defining the presence and ignoring other - "objectively" perhaps as plausible - alternatives. Then the function of memory would have to be described as discriminating against oblivion and remembrance, in the course of which much is forgotten, and remembrance is an exception (Luhmann, 1996). However, at a closer look, it becomes evident that not recalling patterns of information processing alone is always only a precondition for oblivion. At least there is the possibility that these patterns might be considered more relevant on the next occasion - and

\footnotetext{
106 Harald Weinrich (2004) tells the story of Immanuel Kant suffering from dementia who writes down on a sheet of paper that he must forget his servant, Lampe, who has fallen from his grace.

107 For an overview of the issue of deleting see Stefan Hesper (2001), and on ignorance as a special kind of oblivion see Achim Geisenhanslüke and Hans Rott (2008).

108 Accordingly, Georg Kreisler sings the song Ich habe dich zu vergessen vergessen [I forgot to forget you] or Elvis Presley sings I forgot to remember to forget. When stubbornly creating preconditions for oblivion prevents oblivion, this may be called a reflective figure.
} 
under different circumstances. Thus, nothing of the "intellectual property" is lost initially. However, the respectively current nexus influences the further design of path-dependencies, and access, which refers to alternative processing patterns, becomes more unlikely. Schemes, types or scripts that are frequently recalled this way and updated stay relevant as routines of processing information, whereas the recalling of rarely used patterns becomes gradually more difficult. ${ }^{109}$ In other words: we may say that a particular way of dealing with a current situation may be considered obvious; however, another way may be considered "absurd" or at least not particularly "obvious". If one becomes aware of having forgotten something, this is a violation of an availability expectation. Such an expectation refers to information that is supposed to exist as proven by indications or traces. In this context, oblivion constitutes itself regarding the future by addressing a past event of which there is no experience. From drafting future actions, which is confronted with a horizon of incomplete yet expectable knowledge, remembrance is necessary as an intentional action. ${ }^{110}$

If we understand both individual and collective information-processing as a process in which adjustments constantly happen in modifications and continuations, different views in oblivion (II) result. Then oblivion (II) happens as a permanent while at the same time an unnoticed process of neglecting various areas of an experience-induced potential of processing information. The proverbial fading away of memory comes from not recalling, in the current situation, a great number of possible patterns for coordinating behaviour or action. The appropriate structures have not disappeared this way. They are still existent but are not actuated - the path to them may become "overgrown" if it is blocked, for example, by other nexuses. However, that these paths may be reactivated more or less soon becomes apparent when visiting - according to the declarative-reflective dimension of memory - places or relations of one's own, allegedly no longer existent, past and when suddenly, due to the appropriate frame, memories of the "old stories" occur. The situation is similar - in the field of the incorporated-practical dimension of memory - e. g. in the case of a musical piece, the player of an instrument used to master well in the past and which he/she can master again much more quickly than if he/she had to learn it without any background. However, it may also be imagined that both fluency and responsiveness are not as they used to be and that thus the score cannot

109 In certain contexts - such as when the expectation of availability consists of an action routine which has been practiced in the past and furthermore has been made part of one's own biography and cannot be recalled as expected - the insight of having forgotten something is interpreted as unlearning.

110 On this see Alfred Schütz's (1962) essay on "Choosing among Projects of Action", in which he deals with decision-making. 
be played as expected. The stricter concept of oblivion - of oblivion (II) - may well be described by the phrase "out of sight, out of mind", "sight" illustrating current perception and "mind" the current association opportunity. The fact that oblivion (II) may in different ways be connected to oblivion (I) results in problems which will be discussed in the further course of the study.

\subsubsection{Consequences of Restructuring}

If we understand oblivion (I) as a precondition for and oblivion (II) as the process of oblivion, we may consider the condition to precede the process - only in the context of the social because only in exceptional cases or with difficulty the individual consciousness is capable of manipulating itself: an object disappears; thus it can no longer be immediately viewed at or be an element of a reference frame and accordingly does not trigger any remembering definition of a situation. Then the consciousness recognizes and processes other information, and that what has been associated with the disappeared object, as its activation potential or horizon of meaning, retreats. This way, the process of oblivion as a loss of the relevance of individual association may happen, which also holds for the entire association context. Such a process can be demonstrated by the booming of certain topics in the mass media, when a disaster dominates every news channel for a short time, to then disappear again. "Ebbing away", however, is not a result of hearing news of the problem having been solved or having disappeared but of the media no longer being supplied with new news as well as of the topic being replaced by a new one. ${ }^{111}$ If news of a disaster does not only result in compassion but also come along with changed behaviour - such as avoiding certain kinds of vegetables due to fear of these vegetables being infested with germs - changes of classified interpretation schemes can be stated: the consumption of cucumbers and tomatoes is perceived as being risky. However, with the lack of new press reports, these interpretations of danger retreat to the back again - except this kind of vegetable is completely taken off the market. Thus, the fact that cucumbers and tomatoes may be infested with dangerous germs at first extends the concerned schemes; however, in case of a lack of new warnings, this scepticism is eclipsed by other experiences: the schemes of cucumbers and tomatoes are again determined by culinary experiences which are not associated with the fear of health hazards,

111 According to Luhmann, this is a crucial aspect of the influence of the mass media. "The almost neurotic compulsion in the economy in, politics, sciences and art have to offer something new (even though no one knows where the novelity of the new comes from and how large a supply of it exists) offers impressive evidence of this" (Luhmann, 2000, p. 21). 
and consumption goes on as before. ${ }^{112}$ In other words: by way of a mediarelated interpretation, it can be demonstrated how oblivion, as a restructuring of socially classified schemes, happens by way of the disappearance of thus-connected reports from the news of the day. This does not only concern oblivion processes with individuals but can be concluded from a comparison of subjective schemes with socially classified collective schemes. For, whereas among individual people, reservations towards cucumbers and tomatoes may indeed persist, both the mass media public and the political public become less fearful. Then, from the majority point of view, careful people are considered fearful people, and fearful people are eventually considered neurotics.

Furthermore, the difference between oblivion (I) and oblivion (II) can be related to the initially made distinction between object-related oblivion and subject-related oblivion. Deletion, disappearance, ignoring are, first of all, object-related, whereas the process of oblivion is a selectivity problem in the context of individual or social consciousness structures. It seems impossible that any redirection and restructuring of the ways of information-processing by way of the selectivity of memory result in deleted associative connections. The structure of information-processing is maintained, quite in the sense of the psychoanalytical idea of the unconscious or the social-phenomenological idea of the "true-because-motivation",113 without right from the beginning being accessible to the declarative-reflective definition of the situation. Thus,

112 The "ebbing away" of the scheme of danger in the context of a consumption experience happens by the kind of vegetable the consumption of which has at first come to a complete standstill being gradually put on the menu again. If then there does not happen any further irritation, the knowledge of safe consumption eclipses the reservations which are then be forgotten until further notice. However, oblivion does not happen as complete deletion; as soon as there appear messages of a similar danger, the old nexuses are reactivated. This connection between oblivion and possible reactivation may also be described as a loss of trust. Such a loss results from the occasional memory of lost certainty.

113 "[I]n every genuine because-motivation both the motivating and motivated lived experiences have the temporal character of pastness. The formulation of a geniune whyquestion is generally possible only after the motivated experience has occurred and when one looks back on it as something whole and complete in itself. [...] The meaning-context of the true because-motive is thus always an explanation after the event." Schütz (1967, p. 93) distinguishes these because-motivations from subjective meaning in so far as the latter has access to the actual motivations for an action, which are part of an actor's biography, only if he/she turns towards them. At the moment of self-interpretation, however, "they are simply memories and have received their perspective-horzions, their highlights and shadows, from a Here and Now always later than the one in which the project was constituted" (Schütz, 1967, p. 95). Thus, the because-motivations correspond with those imprints into the experiencing individual as being reflectively accessible for his/her consciousness only as construed memory. 
regarding the brain, there is no reason to equate oblivion with deletion or annihilation - and the existing structures are not even lost due to a change of the association system; it is only that currently they are not connected due to the selective activity of the memory. However, the "no connection" diagnosis depends on the existence of a connection that has been interrupted somewhere.

Things are different in the realm of the social. In the case of the structure of information-processing, which can be collectively reconstructed and consists of classified schemes and scripts, deletion due to a change of the ways of information-processing can basically be imagined. This may happen if certain contexts of meaning are no longer needed or have become obsolete, as a result of which the traces indicating them are cleared - such as in the course of cleaning processes. However, these are processes that cannot be started in the short run and without further ado, but usually, they are cultural, quasievolutionary processes in the course of which it has already been forgotten why once one did not want to forget certain things.

Nevertheless - both from the point of view of the individual and the collective - one may leave oneself to the idea of intending oblivion. In this case, usually, oblivion (I) precedes oblivion (II). Thus, in the following, it will be about analysing processual kinds of oblivion (oblivion (II) = forgetfulness) at the various levels of the social first, to then extend the view on oblivion as an action goal, which results in connecting oblivion (I) to oblivion (II) (intending oblivion and making forget). For a sociological argument, this means that the statement that something has fallen into oblivion is exclusively considered from the point of view of the social. By reaching back to the previous assorting achievements, it seems helpful to consider oblivion aspects at the three aggregation or abstraction levels of social phenomena - micro, meso, and macro. This structure is indeed disputed, but it is helpful with making first systematisations.

The micro-level comprises both the level of relations in dyads and microgroups and the individual referring to his/her socially constituted environment, such as in the context of practical, individual action regulation or the problem of subjectively defining a situation. First of all, groups are in focus at the meso-level, the aspect of organisation being at the fore. Finally, the macro-level covers social institutions and cultural practices. Thus, it is about both societies and cultures, if by "culture" we understand a phenomenon going beyond social norms and regulations - as represented, e. g. by national states. Furthermore, we distinguish "automatic" forgetfulness (1), the volitional intention to forget (2), and instrumentally making forget (3) from each other, to then analyse how they affect the three sociological aggregation levels. 
It has already been explained that forgetfulness may be understood not just as a property, or more exactly: as a tendency of forgetting easily and frequently, but also as stating the oblivion process diagnosed for a social entity. As the concept of forgetfulness is always connected to a consciousness-gifted entity ${ }^{114}$ and not to an object which has been forgotten or is supposed to be forgotten, and as furthermore, it refers to a process and not to a state; it is mostly identical with oblivion (II). At the same time, forgetfulness is much in line with the idea of "natural", structural or "automatic" oblivion, as it seems to be in the "nature" of memory to continue or revise associative connections caused by selection processes. This process is experienced as being "natural", as forgetfulness evades the everyday attention of consciousnesses.

Where aspects of such a kind of forgetfulness become apparent in socialscientific theories has already been worked out. At the micro-level (a), "natural" oblivion can be analysed by connecting to the considerations on the subjective experiential context according to Schütz and by connecting to the context of the social reference frame according to Halbwachs. At the meso-level (b) of the social group or organisation, we find indications of forgetfulness, making instrumental use of it most in studies on organisational sociology. Finally, for the analysis of oblivion at the macro-level (c), the range of theories can furthermore be extended by Paul Ricoeur's narration-theoretical works, by Mary Douglas's ethnological studies as well as by the considerations by historians on the issue of oblivion in the field of the historical sciences, such as those of the Annales school.

If the oblivion-theoretically relevant considerations on the subjective experiential context in Schütz and the issue of leaving social reference frames which Halbwachs attributes to oblivion next to each other, the concepts seem to complete each other mutually. Schütz explains the problem of the consciousness turning towards an event with the help of the concept of relevance. In this context, relevance as structurally directing attention is on the one hand caused by an event happening in the environment of the respective consciousness bearer; on the other hand, it is always also induced by the perception apparatus - which has been shaped by experiences made in the course of life. Thus, a change of the experiential context is only possible if an event is declarative-reflectively changed into an experience or incorporate-practically into a routine - thus, if it leaves its impressions in

114 However, this consciousness-giftedness may also be switched off - one is indeed not aware of forgetfulness as a "natural" process, which only makes it a problem for the consciousness. However, discussing this becomes only possible again by way of a reflective view. 
mind or body. As any "undisguised" perception is hardly imaginable and is at best a Weberian-ideal-typical border case, the selectivity of perception is already based on those schemes and scripts as constituting memory. In other words: the relevance-induced control of "view" results from the perceptive faculty as it has developed in the course of life so far. This perceptive faculty is most of all connected to socially communicated experiences. In particular, in the field of thematic relevance, precisely that is moved into "focus", which is described as the social reference frame in Halbwachs. However, with Schütz, this issue can be grasped more fundamentally insofar as Halbwachs seems to be first of all interested in the conditions of the constitution of declarativereflective memory. In contrast, socially structured relevance also includes the incorporated-practical aspect of orientation. At the same time, the frame as a classified scheme is a "handier" concept representing a complex social context. Accordingly, Schütz does not further elaborate any arrangement of topically relevant structures which might be compared to the social reference frame, or more generally, it is just called a "cultural pattern of group life" (Schütz, 1976a, p. 92).

If now oblivion is described as leaving a frame or by a frame becoming obsolete, with Schütz, this must be understood as the lack of a topically relevant stimulus. He deals with this in his works on the stranger and the homecomer, in which - in Halbwachs's terminology - the leaving of social reference frames is analysed. Schütz assumes that the knowledge which is sufficient for coping with everyday life is widely unspecific and inconsistent. However, people living in a specific culture that is the home of such fragmentary knowledge do well despite these limitations. ${ }^{115}$ The stranger - the immigrant in Schütz approaches a different culture whose civilisation patterns he/she does not yet share. To him/her, who is familiar with a different arrangement or with different configurations of social reference frames, many everyday routines of the new group appear dubious, as they cannot claim the "authority of a tested system of recipes " (Schütz, 1976a, p. 96). He/she finds it impossible to remember the past or the experiences of his/her new social environment. At the same time, he/she must successfully participate in its everyday affairs, and for this purpose, he/she can neither make use of the patterns he/she is familiar with from his/her own society of origin, nor can he/she easily connect his/her

115 Schütz (1976a, p. 95) calls this arrangement of culture-spefic frames a fixed and readymade, standardised scheme of cultural and civilisation patterns which has been communicated by "ancestors, teachers and authorities" as an unquestioned and unquestionable guideline for all situations. 
reflective knowledge to the alien environment. ${ }^{116}$ Rather, he/she must appropriate knowledge of the ways of interpretation of the alien cultural patterns.

For Schütz, one such pattern - when it comes to a social group, Halbwachs would also use the frame concept - is the language that goes far beyond the linguistic symbols to be found in dictionaries. ${ }^{117}$ Whereas the already established individual makes use of familiar cultural patterns in an incorporated-practical way, the stranger is forced to include declarative-reflective interpretation processes and thus construes a "social world of pseudo-anonymity, pseudointimacy and pseudo-typicality" (Schütz, 1976a, p. 103). The stranger stays a stranger until he/she completely succeeds with replacing his/her old civilisation patterns with the new ones. Thus, his/her adjustment to the new group is a process of dealing with new civilisation patterns, which eventually may become a matter of course for him/her.

It is evident that by laboriously appropriating new civilisation patterns or new social reference frames, the old schemes are partly provided with new accentuations or modified. Thus, during his/her time in a foreign country, an oblivion process happens due to associative reorientation resulting from adjustment or assimilation. However, it is typical for this kind of oblivion and also this may be stated as being congruent with Halbwachs - that when the foreigner returns to his/her original home, the old, familiar civilisation patterns may be addressed again. What has been completely forgotten in the course of coping with everyday life is soon present again in the course of being reactivated within the old reference system. However, that it is not possible to seamlessly fit in again with the "old times" is dealt with by Schütz in his essay on "The homecomer". Also, this may be read in terms of oblivion theory, if it is, for example, about two peoplewho once were close to each other but were separated and meet again after a long time.

The same things will remain relevant, the same degree of intimacy in personal relationships will prevail, etc. Yet by the mere change of surroundings, other things have become important for both, old experiences are re-evaluated; novel ones, inaccessible to the Other, have emerged in each partner's life. (Schütz, 1976b, p. 112)

116 Right from the beginning, such knowledge is not suitable for successfully interacting with the members of the foreign group (see Schütz, 1976a, p. 98).

117 "In order to command a language freely as a scheme of expression, one must have written love letters in it; one has to know how to pray and curse in it and how to say things with every shade appropriate to the addressee and to the situation. Only members of the in-group have the scheme of expression as a genuine one in hand and command it freely within their thinking as usual." (Schütz, 1976a, p. 101). 
By referencing Ulysses's stay with the Lotus-eaters and by reaching back to reports by the US Ministry of Defence from 1944, Schütz points out that, independently of how the experiences made there are subjectively judged on, a foreign stay comes along with leaving the home patterns. In Schütz's example, the empirically observable consequence of such processes was that 40 per cent of the US American soldiers returning from the war did not want to return to their original jobs and not to their home communities. Schütz explains this by two different worlds where different kinds of anomy are encountered. For the anomy of war, what counts is the bravery of the fighter and his/her ability to gain the upper hand in combat; to this disorder, there corresponds the military virtue of control and discipline towards the "inside". The anomy of civilian life consists of the absence of control and discipline towards the "inside", and not even the structure of "outward" combat is demanded. Then, returning to the old patterns is difficult most of all for those who had been more successful under conditions of the foreign than at home. Like Ulysses's crew, they found it easy to forget the old, and again like in the Odyssey, fitting in with the interpretation patterns and routines of the behaviour of the home environment is difficult.

Schütz describes the process of oblivion because relevant social reference frames have become obsolete in a way that is similar to Halbwachs. In "The Stranger", he demonstrates how subjective classifications - or schemes - gradually change in the course of adjustment processes. Analogously, Halbwachs speaks of oblivion, which necessarily comes when leaving the group and the relevant frames. ${ }^{118}$ However, in Schütz, given the topic dealt with in "The

118 Given the great similarity of the two perspectives, it is remarkable that Schütz (1076c) explicitly distances himself from Halbwachs. In a treatise on the collective memory of musicians Halbwachs (1939) interprets musical notation as the socially conditioned frame of a language of music. The composer's musical creativity, he says, is his/her capability of, within the frame of this language of music, intruding previously undiscovered regions. After all, it is not that musical notation was created for the preservation of music but, vice versa, only the frame of notation allows for creating music. In contrast to this, Schütz is of the opinion that, firstly, an idea does not depend on its nature of being communicable and that, secondly, indeed it is not possible to communicate any musical idea without sounds or notes. Halbwachs, however, wrongly counts on notation only as a communication medium. Although we must agree with Schütz concerning his criticism of the reduction of the frame to the semantics of musical notation, still ther remains - and quite obviously for both authors - the social fact of the frame within which alone (musical) ideas can be provided with meaning. That, as Schütz explains, not musical notation is a precondition for music but playing music together results in developing the frame of a collective memory, (see also Grathoff, 1995, pp. 223-224), would indeed hardly have been denied by Halbwachs when it comes to his understanding of the constitution of social reference frames. 
Homecomer", another phenomenon moves into focus which is easily counted among oblivion. Due to their lack of an immediate relation to each other, both the person living in a foreign country and those remaining home develop ideas of the other, which have not much to do with the respective realities. When meeting again, these perspectives, which have in a way been "alienated" from each other as a result of such pseudo-intimacy and pseudo-typicity, clash against each other. The disappointing experience of not understanding each other, which consists of the others allegedly having forgotten who I "really" am and what I am "really" like, has nothing to do with a loss of knowledge but a separation-induced lack of a shared history. Then the counterpart may be blamed for this, for a kind of light-hearted forgetfulness. ${ }^{119}$

Oblivion at the micro-level of socially constituted subjects and social relations cannot be experienced, as it comes along with continuous processes of adjusting schemes and scripts to social contexts - we may also speak of frames or contexts of meaning. Thus, it looks like the "natural" and not immediately experienceable deficiency of what individuals imagine as memory and what may be described by the concept of the individual stock of knowledge. Oblivion can be experienced only as the consequence of selection processes when the expectation of the availability of knowledge content is articulated. This may be based, on the one hand, on attention having been attracted otherwise and the scheme not showing any current entry-searching; I am moving through the spatial space, trying to remember where I had the key the last time. On the other hand, forgetfulness is experienced with others and through others, if the history of a relationship is assumed there where there is no common past and where everybody used to live with the other in "the heart" but without "seeing" him/her.

That and how social groups forget has frequently been discussed also from a social-scientific point of view. One established motif of social oblivion among groups is the fluctuation of their members as bearers of collective consciousness. ${ }^{120}$ If one does not only look at the individual but also his/her social environment, it is obvious to connect oblivion among the group to the generations of its members. This concludes most of all if the concept of generations is connected to age cohorts, that is, peers, but e. g. also to cohorts of participants who define themselves by joining and leaving the group or their years of membership. Even if a group can establish its own documented or

119 Such synchronisation problems of the memory may also be described by the hysteresis effect of habitus discovered by Bourdieu (see Bourdieu, 199o; Schmitt, 20o9; Wehling, 2011b).

120 Organisational research understands the memories of members to be a relevant subaspect of organisational memory (see Walsh \& Ungson, 1991). 
documents-based frame for its collective consciousness - such as by way of statutes, a "group philosophy", or general principles - still it depends on the individuals it brings together, as representatives and "executive bodies" of these principles. ${ }^{121}$ Vice versa, it must be assumed that the classifications connected to the group's collective consciousness may change due to individual members leaving it. The group as a whole forgets - and this must be distinguished from the forgetfulness of individual members - due to the discontinuation of group-specific communication and practices which the participating individuals realise. This forgetfulness of the group is limited by the fact that particularly meritorious members may, even after having left, be kept in the group's collective mind by way of remembering them and by being adored as symbols of the collective consciousness, which may happen through objectification and thus connected remembrance practices such as memorials, relics or myths. This may also indicate groups being aware of their "natural" forgetfulness, considering this a problem and taking measures against it. How long group members are remembered depends on each group's everyday practical structures. For example, by reaching back to Evans-Pritchard's studies on the Nuer tribe, Douglas demonstrates that the remembrance of the ancestors is maintained as long as it is relevant for giving reason to claims to dowries in the context of marriages - as Halbwachs has it, these are family frames. ${ }^{122}$

Organisations are groups whose goals and cohesion are constituted according to purposively rational or value-rational aspects. ${ }^{123}$ Crucial for this orientation is a clearly defined goal and constant attempts to optimise the ways of achieving these goals. The exact goal is difficult to define due to the different interests brought together by the organisation. ${ }^{124}$ One overarching goal shared by all organisations may doubtlessly be the organisation's survival, which is

121 Karl E. Weick speaks of retention cycles if members of an organisation "store" parts of their organisational experience, to then again recall and communicate them in the context of their tasks. However, he makes the reservation "that only modest amounts of this retained formalized information are injected into organizational thinking, and not much of it is available on a day-to-day basis to affect decisions" (Weick, 1969, p. 214). It is remarkable that after all Weick, who indeed goes at considerable length with his considerations on organisational memory, considers it to be only of modest value, as also he considers it an obstacle for the adjustment performance of an organization.

122 See Mary Douglas (1986) and, fundamentaly, John A. Barnes (1947).

123 In connection to Weber's rationality types it becomes obvious that organsational sociology cannot be taken up in managment research - organisations must be understood both as organisations of work and in the sense of interest organisations (see Schimank, 2002).

124 Organisations are sometimes provided with statutes and documentations where goals are fixed - however these do not cover the functions and informal goals by way of which the roles of individual participants are connected to their commitment with the organization. 
guaranteed by permanent adjustment to the environment. ${ }^{125}$ Although the forgetfulness stated for the social micro-level and, generally, for social groups is also encountered in the context of organisations, under the perspective of adjustment, the forgetfulness of the organisation seems to be an ambivalent process. Accordingly, adjustment to a changing environment requires a high degree of flexibility and openness which is hampered by an all too strict orientation, in the form of established structures, at one's past. Against this background, a certain degree of oblivion seems to be a precondition for adjustment and organisational learning. ${ }^{26}$ Furthermore, precisely the rational organisation is characterised by highly artificial and differentiated selection mechanisms. Seen this way, control of the group memory is an advantage of the organisational structure compared to the disorganisation or alleged primordialism of unorganised groups. This does not refer to the contents of the archives of organisations, which develop from documenting processes in the form of files; after all, even documents are open to interpretation, and reading them as well as including the information found there into the organisation's communication may also result in oblivion. ${ }^{127}$ More important is the practice of documenting with the help of files and, e. g. the establishing of a hierarchy of statuses and positions that connects selection tasks to certain positions.

Additionally, there are ways in which organisations succeed with safeguarding successful processes against their own forgetfulness. In this context, Luhmann points out to organisational programming: utility programmes fixate all those premises for decision-making which are needed for achieving organisational goals. On the other hand, conditional programmes are used if decisions about rare yet vital situations must not be forgotten. As a selection structure, utility programmes determine or coordinate the "everyday business"

125 The interaction between the two future prospects of "goal achievement" and "continued existence" may, in particular in the case of organised interest groups, result in the continued existence of the organisation even if the goals defined by the statutes have been achieved. This is the case if the structures established in the course of goal achievement have been consolidated to such a degree as to allow for orienting at new goals. This way the original goal orientation becomes irrelevant and may be forgotten or left to the organisation's historiography.

126 Already at an early stage, James G. March (1972) pointed out that organisational memory may become the enemy of organisational learning.

127 On this see Niklas Luhmann (2000, p. 16o) who points out to the fact that files do not only "organise" remembrance but also oblivion and that, when being read again as texts, they may as well motivate for conformity instead of deviation. Communicating decisions in writing creates its own time, a time for self-mobility, for dynamic stability; and empirically undeniable obstacles for innovation may be due to completely different reasons, such as the "organisational culture" which is not recorded by the files. 
of an organisation. Conditional programmes appear as a memory second order, as they are kept ready for specific situations (Luhmann, 20oo, pp. 275-276). The organisation "knows" about an avoidable loss of control when it refrains from continuing proven ways of proceeding. Then it runs the risk of failing to adequately adjust to a changing environment (Dimbath, 2009). Against this background, it seems to be "rational" to orient the selectivity according to the organisation's experiential context, past or "history".

Several research works are dealing with the "natural" or "automatic" forgetfulness of organisations. Their diagnoses are different from each other, each according to the theoretical background and empirical approach. For example, Walsh and Ungson state that when it comes to forgetfulness among groups due to the change of members, the members contribute considerably to organisational memory. This is more likely the longer somebody has been a member of the concerned organisation. The knowledge of organisational processes and corporate identity connected to the organisation's culture depends essentially on staff members whose "everyday business" is precisely this. If a long-standing staff prevents certain kinds of fluctuation-induced forgetfulness, it hampers the acquisition of younger members of the organisation whose innovating potential will stay unexploited due to all too strictly insisting on established structures (Walsh \& Ungson, 1991). According to Christopher Pollitt, those organisations, on the other hand, which believe in achieving a high degree of adjustment by placing much value on younger staff and a high degree of fluctuation run the risk of a loss of memory, as the members have neither the possibility to extend their task-specific experience nor of passing it on to others - each member starts from scratch. ${ }^{128}$ Thus, the extent of organisational forgetfulness depends on the extent and fluctuation or the consistency of its membership structure. However, it would certainly be superficial to state that long-standing staff shows no forgetfulness at all.

As it must be assumed that referring to established social classifications in the sense of collectively binding schemes and scripts run more comprehensively and undisturbed, specific adjustment processes, such as product innovations with enterprises, are frequently connected to existing types or traditional knowledge. This may result in the innovative aspects of new processes not entirely included in the organisation's experiential context. Thus, forgetfulness happens because new knowledge cannot be remembered, and it is diagnosed if the organisation finds that recently acquired knowledge has been lost again and that a successful process can neither be repeated nor transferred into

128 Pollitt (2009) reports this by referring to studies on temporary contracts with boards and ministries - a phenomenon which he calls a decline of the bureaucratic career. 
the company's routine. Apart from this incapability of adopting innovations caused by memory-specific selection mechanisms, the well-known problem of knowledge becoming degenerated and simpler in the course of action routines must be called a kind of organisational oblivion. This problem, reconstructed as another kind of oblivion, is illustrated by Pablo Martin de Holan and Nelson Phillips by the example of a restaurant that has been granted its third Michelin star and has thus advanced to the worldwide elite of restaurants. Three years later, it is incapable of maintaining the high standards of its service of the past, as the knowledge of the thus required refinements has "fallen by the wayside" (de Holan \& Phillips, 2004a). From the point of view of theories of organisational learning, this is a specific kind of unlearning. ${ }^{129}$

Forgetfulness among groups and organisations is due to "ordinary" structural change, which mostly evades the perception of the historically uninformed observer who is also under practical pressure to act. However, this forgetfulness may be made a subject of discussion if problems interrupt the course of everyday routines that are identified as being known due to certain traces or starting points. The rational organisation tries to avoid such situations by purposefully organising those selection mechanisms as constituting its social memory - such as programming. Only more recent studies on organisational learning have revealed the Janus-faced character of such a strategy. Sometimes, it results in radical destructuring measures concerning the organisational memory, which, as adhocracy or post-bureaucratic organisation, focus on criticism or run the risk of overshooting the mark of better adjustment and forgetfulness main principle of their activity. ${ }^{130}$

Given the social macro-level, it must be stated that the as yet negotiated kinds of forgetfulness culminate at this level, which is the highest level of aggregation and abstraction, first. Thus, what has been worked out so far is valid also there, and much of what has been diagnosed in the context of relations at the micro-level, for group and organisations, is also true for institutions, societies and cultures. However, there are also aspects of forgetfulness that are located most of all at this level. More specifically, they become apparent from studies on the forgetfulness of institutions and the forgetfulness of cultures, particularly given historiography in the context of national states and societies. ${ }^{131}$

129 In this context it would make sense to discuss if unlearning must be equated with oblivion. On the problem of unlearning in the context of knowledge management see also Stephanie Porschen (2011).

130 On post-bureaucratic organisation see Pollitt (2009) - adhocracy is counted among the situative approach of organisational research (for an overview see Kieser, 2001).

131 However, this is also true for group or organisational history. Against this there works the project of oral history which turns precisely against forgetfulness caused by the 
What Mary Douglas describes as institutions controlling the perception of individuals within a particular space and at a certain time is what Ludwik Fleck and Karl Mannheim called the thought-style (Denkstil). ${ }^{132}$ These are comprehensive typologies that are valid for large groups. The thought-style of a specific time implies a particular kind of forgetfulness insofar as its particular selection mechanisms systematically exclude several alternative points of view. This exclusion happens on the one hand at the moment of (public) perception, by way of ignoring, and on the other hand also because of the opportunity to change, by way of political influencing, existing classifications which are understood as collective schemes. It must thus be stated that everything looking alien against its background is less likely to be transferred into experience regarding the forgetful institution. Many indications of something new are either overlooked or not "kept in mind". Forgetfulness is admitted - usually at the political level - if one must acknowledge to oneself that one has not recognised the signs of the times.

Forgetfulness and thus hampering learning due to being fixated on the past prevents new things from being adopted; however, forgetfulness appears also vice versa, as learning may also be prevented by not learning lessons from the past: as it is well known, who is not ready to learn from history will be forced to repeat it. ${ }^{133}$ Through this warning, the significance of the science of history for politically defining the present may be particularly emphasized. Historian Lucian Hölscher, on the other hand, believes that contemporary historical awareness is characterised by oblivion to a high degree. Any historiography, he says, happens in the mode of declarative-reflective memory. Although it aims at researching how past events "actually" happened, all it can do is reach back to the memories of others or sources. From the fact alone that the historian believes - due to his interpretations being a construct - to know more about historical events than the total number of all sources tell, one can conclude that he/she must assume comprehensive social oblivion. Any kind of social

unreflected reproduction of existing power relations in studies and in the course of working with sources. The debate on history, historiography and memory happens in view of the macro-level - this really traditional debate starts with the dispute between Halbwachs and Marc Bloch, to be found again in Jacques Le Goff or Pierre Nora (on this see J. Assmann, 2005; Gierl, 2005) as well as in Helmut König (2008).

132 See Mary Douglas (1986) who refers to the works by Polish physician and science theoretician Ludwik Fleck (2012) and to memory researcher Frederick C. Bartlett (1977). However, the concept of thought style, which she attributes to Fleck, is a couple of years older and might go back to Karl Mannheim who connects to Max Scheler.

133 This statement, which is certainly dubious from the historian's point of view, goes back to philosopher George Santayana (1905). 
memory necessarily ignores many facts that are not or cannot be made a part of remembrance. ${ }^{134}$

To sum it up, social forgetfulness can, on the one hand, be concluded from different kinds of amnesia concerning individual consciousnesses but potentially creating collective effects, after all. If with Halbwachs or Goffman, the social order is described as a system of different reference frames, and if one follows Schütz's relevance theory, oblivion at the level of the individual comes along with the loss of orientation-providing material or immaterial knowledge elements, meanings or important facts. To the degree to which individuals can no longer identify certain frames, they will - quite automatically - forget many aspects of the thus connected contexts of meaning.

Whereas at the macro-level this kind of forgetfulness can also be identified with regard to groups, we can identify further variants of an oblivion-induced loss of knowledge with collectives. The collective memory of the group structures both its goal and its "identity". During centripetal periods - such as rationalisation programmes in the case of organisations - both may be shaped more clearly and be provided with higher relevance; during centrifugal periods, it may rather be weakened. On the one hand, such a weakening may be connected to individual actors leaving together with their significance for the group's cohesion; on the other hand, it can be connected to a decline of potential in the context of maintaining social order ("sloppiness"). At the macro-level of socialised large groups and institutions, there happens a kind of oblivion that is self-induced due to the relieving effect of habituated rules. Institutions rule out alternative solutions that the individual or the group might choose but are actually "unimaginable" because of the given order.

As the second kind, by wanting to forget, a kind of oblivion is described, which may also be called intending what cannot be intended. ${ }^{135}$ This is about the intention to forget a burdening experience volitionally. In contrast to "quasi-natural" forgetfulness, the intention to forget develops from a combination of the two kinds of oblivion (I) and oblivion (II), as it is about creating favourable conditions for starting a "natural" oblivion process. Such attempts

134 "This does not refer to the trivial fact that any recalling of a fact requires the latter to have been forgotten and, vice versa, any oblivion to previously-having-kept-it-in-mind; but rather to the fact that by remembering obviously there appears a shift of the remembered fact, a suppression of the original event in favour of an event which has been modified afterwards. This shift, which also includes oblivion, happens involuntarily and inevitably." (Hölscher, 2009, p. 106)

135 Paradox figures in the sense of intenting something which cnnot be intended are collected by Jon Elster (1983) in his study on the Sour Grapes. Studies in the Subversion of Rationality. 
at manipulating the consciousness can be identified both at the personal and at different social levels. Just the same, at the level of individual consciousness, particularly in the context of dealing with traumatising experiences which as they generate persistent memories - can no longer be suppressed ${ }^{136}$ and forgotten, such attempts appear to be as necessary as they are hopeless. What in view of the individual consciousness appears as being complicated if not impossible is more likely in the context of the social relations: for example, one may (commonly) decide to forget about an injustice by way of forgiving and reconciliation. Thus, the intention to forget must be analysed from two points of view: on the one hand as organised self-delusion or manipulation, and on the other hand as manipulative social engineering. In any case, it is about the attempt to reduce dissonance by not allowing memory-specific, problematic schemes, classifications or associations to interfere with the process of constructing and defining current situations. This requires steering and control, consisting e. g. of reorganising social reference frames and removing memorytriggering objects or interrupting connections.

Given the one-sided or mutual desire to forget irritations for a relation, the phenomenon of wanting to forget may be analysed at the social macro-level (a). In this context, at the meso-level (b) of social groups and organisations, collectively binding taboos and negotiating oblivion agreements move into focus. Also, at the macro-level, it is possible to diagnose an intention to forget in the context of certain ways of collective silencing.

The individual's desire to forget has produced the result that an ars oblivionalis was already announced in antiquity, apart from the ars memoriae. Such an art of oblivion may at first refer to dealing with one's own experiences. What regarding oblivion (II) and the already mentioned considerations by Umberto Eco (1988) is considered to be at least difficult, although sometimes it is attempted with the help of measures in the mode of oblivion (I), appears as a civilising step in case of social interaction or relation. This step serves to maintain the stability of the self, the belief in the certainties connected to the community, and the further existence of a relation that has become problematic. Such an ars oblivionalis is not only suitable for making or keeping pace within the social group, but it also works against not-being-able-to-forget caused by traumatisation. By way of a self-objectification that uses the knowledge of

${ }_{13} 6$ Here the term "suppressing" seems to be appropriate - however at first without its psychological meaning. Experiential knowledge connected to violations is not overwritten or deleted and is preserved as a warning reflex, without any possibility of replacing it by alternative or new patterns for defining a situation in the course of further everyday experience. 
social oblivion, such a desire to forget by the individual can be made plausible. A number of strategies by which subjects attempt to outwit themselves and their urge to remember result from this.

Only at first sight intended oblivion from the point of view of the individual consciousness appears as a purely psychological issue. The desire to forget appears for the first time when an experience that is perceived as hurting the soul either proves to be persistent, by again and again and on allegedly inadequate occasions become an element of a situation ${ }^{137}$ and usually also of the definition of this situation. On the other hand, a desire to forget can be identified if an experience - as a weaker variant of trauma - is supposed to sooner fall into oblivion (II), as it is perceived as mortifying. Furthermore, the desire to forget alone may result in the thus connected associations being increased and thus, after all, also to this "memory" becoming persistent. This holds most of all for memory effects that are not dealt with in the declarative-reflective way, which in trauma research are understood as incorporated protection reflexes. According to psychoanalytical theory, as a result of dissociation, suppression or inhibition, they cannot be reflected on and thus sometimes appear in the form of neuroses (see Schacter, 2001). It is not easy to imagine that a burdening experience can be thought without a social-biographical frame. Thus, traumatisations have to do with social relations, in the context of which the persistence of experiences, which have solidified to experience, can first of all be explained by the strong involvement of the incorporated-practical memory dimension. ${ }^{138}$ The reference to the body memory is an indication that many therapy attempts start from the body. Thus, the strategy of drowning grief in alcohol or different kinds of escapism, such as the consumption of media or drugs, may be understood to be a body technique, just like behavioural therapy, in the course of which situative-problematic routines of behaviour are supposed to be "overwritten" by alternative routines of behaviour. Also, more recent methods, such as pharmacological products or the technique of retrieval-induced forgetting or of eye-movement-desensitizationand-reprocessing (EMDR), aim to change the body in the sense of fighting

137 Thus seen, the intention to forget - like in the story about Kant and Lampe - is already a memory-specific selection mechanism which, by way of assessing if something has been forgotten, constantly updates, by way of remembrance, the object which is supposed to fall into oblivion.

138 Accordingly, neuro-scientific oblivion research attempts to make trauma-related imprints less emotionally burdening (see Henry et al., 2007). 
symptoms. ${ }^{139}$ Other strategies are e. g. changing the environment in the sense of a radical change of frames, which is connected to the hope of no longer seeing anything that might remind of the past. ${ }^{140}$

However, not only the trigger of the trauma is social; also, the experience of being burdened may - at least partly - be traced back to a discrepancy between subjective experience and social horizons of expectation. ${ }^{141}$ Trauma is a kind of suffering that is due to an irresolvable dissonance of two realities, as Schütz describes it in the case of the homecomer. This is a reciprocal oblivion assumption coming along with a fundamental loss of recognition which - given the fact that there is no opportunity for discussing it in a declarative way - is "acted out" exclusively or at least first of all in a bodily way. Then, the consequence of maladjustment to the home society caused by the experience of completely different relevance in a foreign environment may first be the desire to forget again about these foreign relevancies. As this is not possible, and as the effects of the new schemes on each current definition of a situation at home cannot be prevented, there develops a therapy case in the sense of Berger and Luckmann. They understand therapy to be society safeguarding itself against institutionalised definitions of reality by outsiders. ${ }^{142}$ In other words: from the point of view of society, this kind of therapy - which is voluntary and must be wanted by the individual due to his/her own insight - serves for reintegrating outsiders and for providing the concerned environment with a suitable

139 On this see the short overview in Christoph Lau, Peter Wehling and Oliver Dimbath (2011) as well as the elaborations on intentional forgetting in Golding and MacLeod (1998).

140 Also, here, as one out of many examples, we may refer to the failed attempt by Max Frisch's fictional character Ludwig Anatol Stiller. The new beginning in America represents such an oblivion strategy.

141 Thus seen, taking the perspective of the victim in stigmatisation processes is always connected to persistent remebrance; thus, labelling or stigmatization also appear as a memory-specific selection process.

142 "Such a conceptual machinery permits its therapeutic application by the appropriate specialists, and may also be internalized by the individual afflicted with the deviant condition. Internalization in itself will have therapeutic efficacy. In our example, the conceptual machinery may be so designed as to arouse guilt in the individual (say, a heterosexual panic'), a not too difficult feat if his primary socialization has been even minimally successful. Under the pressure of guilt, the individual will come to accept subjectively the conceptualization of his condition with which the therapeutic practitioners confront him; he develops 'insight', and the diagnosis becomes subjectively real to him." (Berger \& Luckmann, 1967, p. 131) 
interpretation of deviating behaviour. Thus, one has oneself been brought "back in line". 143

Apart from these oblivion strategies of the individual, a dyadic intention to forget can also be identified. The latter becomes evident by phrases such as "letting somebody get away with something" or "not being unforgiving". Thus, tolerance and calmness in close relationships serve to maintain the intent to be forgiving - such as ignoring and forgetting - despite frequently being irritated again. This is possible if one decides not to place too much weight on disappointed expectations and prioritise the continuation of harmonic togetherness over a threatening conflict. This is an everyday practice of more or less intended oblivion, by quite purposefully - in the mode of oblivion (I) - preventing disappointing experiences from becoming solidified, in order of not hampering oblivion (II), if possible. One established way of clearing up irritations and avoiding irritation of a relationship to be continued by a sequence of mutual insults and revenge is discussing the problem. In severe cases, this may be connected to the goal of achieving reconciliation and forgiveness. ${ }^{144}$ In everyday interactions, this may be accompanied by trivialising or at least minor conflict-laden semantics if it is about clarifying "misunderstandings".

The phenomenon of wanting to forget among groups is dealt with by ethnologic research on tabooing. One well-known figure is maintaining "inner" peace by not mentioning past conflicts and their consequences. In this context, groups do not only show a tendency of being silent about bad experiences but also of preventing any communication about them not to endanger social cohesion. This may go as far as denying the victims any kind of recognition because any punishment would put the community's basic principles of coherence as well as its security fiction into question. However, like at the micro-level, here two perspectives should be distinguished: firstly, the group's self-reflective orientation at "inherent" oblivion, called a (self-)technique

143 Not only since the occurrence of globalisation processes the globetrotter is contrasted to the individualised type of the placeless person - such as the urban nomad who oscillates between cultural relevance systems, who is capable of communicating everywhere but has no home. In late-modern society this can be tolerated in the context of cosmopolitan interpretation patterns or simply because he/she cannot be considered a guest or a foreigner. If, however, the placeless person gets confused or revolts because due to his/her experience he/she is able to compare, he/she may soon be declared a therapy case. In case of doubt, he/she is expected to forget the other, with which he/she is also familiar, and to credibly signalise belonging and loyalty.

144 Paul Ricœur discusses the difficult relation between forgiveness and oblivion and comes to the conclusion that "forgiveness is a kind of active oblivion" (Ricœur, 2004a, pp. 144145) which must be distinguished from passive oblivion. Forgiveness requires common remembrance, to this way create the preconditions for common oblivion. 
above, may be emphasized. Secondly, however, also the relations groups have to their environment must be considered analogously to discussions at the micro-level. The communication between groups may happen both by intended oblivion and expecting others to forget.

The connection between tabooing and oblivion has already been addressed elsewhere. In the case of far-reaching inner troubles, a group may decide to impose a taboo on past conflicts. Indeed this is not the only reason for imposing taboos, which may also derive from power-political intentions. Furthermore, taboos on claims to revenge as known from history and being connected to oblivion - or amnesties - are first of all documented at the social (macro-) level. However, the ban on mentioning past events might endanger the group's cohesion and is also known from families or village communities, such as when a "dark secret" is kept.

It seems to be that the self-location of oblivion with organisations is less problematic. Those scenarios and theories as developed in the context of unlearning as well as theories of organisational learning conceptualise oblivion as a vital strategy of organisational adjustment and explicitly include it into their management doctrines. For example, international cooperations, takeovers, or just the reorganisation of product lines sometimes result in the opinion that the "culture" of an enterprise must be fundamentally changed. Pablo Martin de Holan and Nelson Phillips describe this by the example of the cooperation of a Western hotel chain and Cuban enterprises, in which oblivion processes were purposefully started in the interest of synchronising routine processes (de Holan \& Phillips, 2004b). Strategies of oblivion may be implemented by first uncovering the concerning routines and programmes, to then explicitly taboo and exclude them. In practice, this may be achieved first of all by introducing new programmes and ways of communicating and processing. However, such a reorganisation may also require an exchange of staff.

For the interaction between groups, it is fundamental to preserve certain aspects of a shared past, however others not. Events connected to group relations that are not helpful for each individual group or the cooperation of several groups are practically not remembered. In this context, it must be remarked that even hostility between groups may definitely be functional for each group's inner cohesion and may thus be maintained. ${ }^{145}$ In view of groups

145 On this see the classical distinction between ingroup and outgroup, the campsite experiments by Muzafer Sherif (1969) or more recent approaches of othering or changing (see Reuter, 2002). In this context, for example the remembrance policy of school curricula is a crucial contribution to group-specific integration. Education creates a cultural reference frame of its own kind by way of which integration may happen across groups or milieus. By providing canonized knowledge as an aspect of collective identity it is possible to 
in general, also this is very close to the problems at the macro-level of the social, which are going to be negotiated further below. There, mutually related oblivion among groups may happen, e. g. peace-making processes and mediation, if forgiveness is granted and accepted.

In inter-organisational communication, oblivion processes are regulated by law, the media or the market. ${ }^{146}$ Jurisdiction allows organisations to formalise their conflicts and to distinguish between conflict theatres and cooperation relationships. This way, the organisational memory is provided with selection opportunities different from those of the social group. However, there are cases in which a conflict happens at the level of "organisational cultures", and strategic oblivion is difficult to implement. ${ }^{147}$

One variant of intentional-volitional oblivion or of preparing and enabling for forgetfulness at the social level is the practice of silencing. At first, silencing follows principles that are similar to group-related oblivion demands. Additionally, however, the expectation that certain events are not mentioned is no longer exclusively based on group-related coherence mechanisms - such as according to the understanding of mechanical solidarity in Durkheim. Instead, it is about an initially unclear connection between declarative-reflective and incorporated-practical memory, which must be considered volitional because there is a tacit agreement not to mention a certain topic. However, this is different from forgetfulness due to the apparent functionality of silencing, which once revealed - may easily be attributed with meaning. ${ }^{148}$ In the following, we will distinguish two kinds of silencing: silencing towards the inside and silencing towards the outside.

A special kind of institutionalised tabooing as hope for oblivion is being collectively silent about one's own shame. ${ }^{149}$ This becomes an institutional topic

cover up the sometimes fundamental sub-cultural differences between social groups or milieus so that they may be forgotten (see Georgi, 2006).

146 However, these are macro-phenomena - money as a system medium does not remind to anything except to itself. Yet still, the memories of the economic system find expression by the language of money (Baecker, 1987, pp. 528-529).

147 In this case we must think of take-overs of enterprises and unequal mergers in the course of the process of economic concentration, when the collective identity of a group is damaged in the course of the problematic synchronisation of organisational cultures and, as a consequence, there are failures.

148 The many examples of amnesty-related oblivion in antiquity rather appear as mesoinstead of macro-phenomena, as it is about peace both among and between groups - it may as well be based on a volitional desire to forget, resulting from a home- or foreignpolitical decision-making process.

149 Here, Aleida Assmann distinguishes between the memories of the victims and the memories of the vanquished. "Traumatic experiences of suffering and shame find it difficult to 
if silencing is also legally normatively backed, legitimated and thus sanctionable. Examples of this variant of the group-related intention to forget are found in the history of coping with dictatorships and state terror in South-American societies. There one gets the impression that the whole continent suffers from an "illness of oblivion which has simply lifted authoritarianism by way of amnesia" (Traine, 2009, pp. 96-97). Sometimes this happens by hardly listening when charges are filed, and the atrocities of the past regime are lamented. This is partly due to the practice of protecting former office bearers from legal persecution by granting them immunity or amnesty; sometimes however, there are also informal inhibitions when deciding about charges in a legally correct way. ${ }^{150}$ Such inhibitions must be attributed to a macro-structural-volitional kind of oblivion if there is a tacit agreement about too much "probing" being undesirable. Apart from legally fixed deadlines for amnesty, we must assume an implicit expectation of a limitation of actions regarding past injustice. This is different according to culture and has as yet hardly been empirically researched. After this has expired, guilt assignments are no longer accepted, and sometimes accusers are even negatively sanctioned. One starting point for this is the sequence of generations when the following generation replaces an "ageing" generation or entelechy of generations. Then the relevancies of the old generation are derided or discredited as being old-fashioned, outdated or outmoded. ${ }^{151}$

Being silent about a past that is interpreted as being problematic may be based on two motivations. On the one hand, giving up on discussing the topic results from the abolished or collapsed old order and the thus resulting necessity of fundamental reorientation. This concerns new political-moral norms and values as well as focussing on the reconstruction of whatever kind. On the other hand, there may appear several interest groups within one society, in the

be included in the memory because they cannot be integrated into a positive individual or collective self-image" (A. Assmann, 2002, p. 187).

150 The problem of silencing practiced both by perpetrators and victims in Spain is dealt with by Beatrice Schlee (2009).

$15^{1}$ Connecting to Mannheim's (1952) concept of generations, here we must distinguish between a concept of generation in the sense of age cohort and the (large) group-related shape of a generation. This distinction is necessary for more detailed analyses, as the definition of new relevancies is not made by the entire age cohort but by a sub-group which Mannnheim defines as a generational unit which, against the background of its own position within the generation, succeeds with unfolding definatory power. First of all, one delimits from the predecessor generation - only secondarily it is about coping with competing peers. 
context of which the more powerful ones enforce their own ideas and intentions of transparency or investigation. ${ }^{152}$

Volitional oblivion with large social formations such as nations, cultures, peoples or tribes looks back to a long history. It is reflected by the symbolic acts of peace-making. A mutual arrangement expressing the desire to forget about a past conflict for the sake of a future relationship which is supposed to be different both at the level of identity and the formal-legitimate level may be illustrated by the phrase "to bury the hatchet". 153

A very comprehensive debate on the problem of institutional oblivion concerning the mutual relations of large groups - and this can only be hinted at here - happens in the context of Holocaust remembrance. ${ }^{154}$ Whereas dealing with the crimes of National Socialist Germany has resulted in inherent accounting in the context of selective acts of remembrance, at the international level, there has established a specific, institutionalised way of memoryspecific selection in the context of processes of remembering, recompensing and accounting. On the one hand, these processes have been accompanied by accentuations of remembrance to prevent any kind of oblivion which might be expected for the political and social practice, which sometimes finds expression by saying "forgiving - well okay, forgetting - never". 155 It is thus about inhibiting forgetfulness. On the other hand, the institutional way of accounting for the past always comes along with specific aspects of oblivion in the

$15^{2}$ As a typical example of the former case, sometimes the German post-war society is given, although the protest of the 1968ers aimed precisely at the unbroken activities of old NS elites. It is at least institutions-theoretically imaginable that a fundamental change of fundamental social structures is at first a subject of silencing. As a matter of fact, in the case of Germany we may suppose a mixed form of paralysis of the vanquished, tabula rasa and the notoriously forgetful memories of the perpetrators (see Aleida Assmann, 2002; Giesen, 2004). Concerning the latter case, the problems of coping with the Chilean dictatorship are given as an example (see Wehr, 2009).

153 Overcoming the "hereditary enmity" between Germany and France may be given as an example of a mutual adjustment of remembrance, in the course of which hostile knowledge stocks and traditions are left to volitional oblivion, as they are considered to be no longer fashionable.

154 In view of remembrance policy in the context of European unification, Claus Leggewie even speaks of "Europe's negative founding myth" (Leggewie, 2011, pp. 15-16).

155 Forgiveness by Vladimir Jankélévitch may be read in this sense - it starts with these words: "It is legitimate to have a grudge against criminal for twenty years, but after the twentyfirst year, one becomes rancorous! With full rights and from one day to the next, the unforgivable is thus forgotten. What had been on for unforgivable until May 1965 has abruptly creased to be in June 1965 . It is indeed necessary to set a date, is it not? And so official forgetting begins tonight at midnight." (Jankélévitch, 2005, p. 35) 
context of which a way of remembrance is ignored state actors have agreed to not want.

Also, with changes in the relations between nations, we must diagnose aspects of volitional oblivion. A comprehensive change of how to deal with cross-national memories is described, e. g., by theoreticians of second modernity. For example, Daniel Levy and Natan Sznaider demonstrate his reception in different national states in their discourse-analytical reconstruction of the debate on Daniel Goldhagen's book The Holocaust and memory in the global age (Levy \& Sznaider, 2006). According to their estimation, the success of this study, which is disputed among historical scientists, is the result of an authenticity deficit of (US American) Holocaust remembrance. It is thus an effect of institutional oblivion at the global level, as the experiences of victims had been ignored by historical accounting, and the victims could now make themselves heard. ${ }^{156}$

However, even beyond accounting for a bad past, under conditions of increasing globalisation, a degree of institutional adjustment must be assumed, which, in the individual case, may even happen in the form of a fundamental break. This begins with the destruction of indigenous cultures in the course of colonisation and Westernisation and goes as far as to allegedly "soft" adjustment processes such as McDonaldisation, in the course of which cultural particularities may be suppressed and forgotten over time..$^{157}$

${ }_{15} 6$ For this, the tension between sources-based historiography and statements by contemporary witnesses is crucial. The memories of contemporary witnesses are indeed very much authentic, however frequently such memories are mistaken - on this see Aleida Assmann (2007) as well as the critical debate in Alexander von Plato (1999). In the context of volitional oblivion at the level of collectives or collective identities this may result in considerable conflicts about interpretation.

157 The statements by German politicians giving expression to their readiness for different steps towards European unification may be understood as argumentative routine in the service of anti-traditionalism and anti-particularism and as resulting from the history of Germany's coping with its own past. That "the" Germans seem to adhere so little to their national symbols, their Deutschmark, their grammar schools, their universities, is perhaps due to the "zero hour" experience. Thus seen, the German post-war society is ahead of many of its neighbouring societies, as far as the intention to forget traditional and habituated structures is concerned. That still in many fields nothing happens except statements becomes obvious everywhere where institutions do not bow to a sometimes short-sighted desire for change. For, whereas on the one hand institutions function as oblivion generators - in the sense of ruling out any alternative action - by their stubbornness they frequently prove to be much more complex than expected by reformers. The phrase "never change a winning team" indicates, among others, that change management runs the risk of not taking all steering-relevant aspects into consideration, that is of forgetting some of them. 
The intention to forget something proves to be a civilisation-related aspect of the oblivion concept, as it demands the actors to give up on satisfaction and triumphalism - of whatever kind. In its pure form, as a civilisation-related precondition for oblivion, it marks a difference to a discipline-enforced desire to forget: the civilisation-related kind is connected to the figuration of social (interest-)groups. It is based on the insight that, by reconciling past conflicts through mutual benevolence, a new beginning and a start or continuation of the social relationships are possible. In the case of the discipline-enforced kind, the emphasis is more on the power-related and sometimes implicit expectations of society the subject is supposed to meet. Here, the aspect of constraint by others is more emphasized, whereas in the former case, selfconstraint in view of being empowered to decide freely is weakened. ${ }^{158}$

In the context of social groups and political decision-making, this motif appears as promising progress of civilisation if the decision for tabooing and purposeful silencing has been made in a participative way. To prevent claims to justice from being limited, this must have been preceded by commemorative constructions of memory - such as in the sense of an at least symbolical yet authentic recognition of the bad past and thus connected reconciliation.

The third dimension of social oblivion is oblivion initiated by third parties, i. e. making forget. In this combination of oblivion (I) and oblivion (II), actors are assumed who make others forget something. For this purpose, they must first assume a "natural" kind of forgetfulness in the sense of oblivion (II) based on a topic that is not discussed and perceived and will lose its relevance. Like in the case of self-delusion in the context of wanting to forget, this may be supported by removing the concerned subject or preventing and prohibiting any search for traces. This is not only about erasing traces indicating a deed that has not been punished. Rather, the focus is on actions aiming at an object of knowledge available for individuals who are supposed to be enabled or obliged to oblivion. It is thus about the social organisation of selectivity in the sense of staging and deciding about relevance. In this context, launched oblivion projects may aim to cope with interactions that are expected to be problematic or aim at deleting a past event from the framework system that allows for collective remembrance. Also, the following elaborations are structured according to making forget at the social micro-level of subjects and interpersonal relations

158 Norbert Elias (1981) and Michel Foucault (1977) by their concepts of civilising and of disciplining point out to the figure of the transition from self-constraint to constraint by others. The project of a comparative debate on this theoretical motif has hardly been explicitly started for the time being. 
(a), to the meso-level of social groups and organisations (b) as well as to the question about the social macro-level which comprises oblivion policies (c).

The creation of conditions which are supposed to support oblivion (II) with others is at first in line with social action in the sense of Max Weber: it is about anticipating how probable memory is with others and, by way of one's actions, about tolerating or neglecting, about taking care that this probability will decline. ${ }^{159}$ The manipulation of memory may at first be strived for and sometimes indeed be achieved at the neuro-physiological or psychological level by medical-therapeutic intervention. This may be imagined to be done with the help of mind-altering medication or brain manipulation. However, what in the context of the individual consciousness looks like dystopic science fiction, is not uncommon in the practice of social action and thus in the field of social memory, if it is about methods and strategies of "shaping" or manipulating both individual and collective memory. ${ }^{160}$

Erving Goffman's frame theory allows for shedding light on such aspirations. As well-known, the change of primary frames happens by way of modulation and deception. Deception consists of an actor's attention being purposefully directed at a particular frame, whereas the other (deceiving) actor refers to his/ her action to a different frame. To this, there belong all kinds of purposeful deletion, destruction, hiding or obscuring against the background of not revealing the concerned measures - Goffman calls this collusion. ${ }^{161}$ Deception, aiming at making others forget, produces the effect that the view of an object which is considered to be "objectively" emergent is blocked. This way, any association of the thus connected horizons of meaning becomes improbable. Then the deceiving actor hides the object in question; for the deceived person, on the other hand, it is non-existent. Even if for him/her the object is interpretationand thus action-relevant because he/she is familiar with the appropriate possibilities to connect, he/she will not be able to address them because the frame he/she is provided with does not offer any indications.

159 See the sociological basic concepts in Max Weber (1979).

16o An impressive example is the collective deletion of memory as presented in the film Dark City by Alex Proyas of 1998.

161 In case of a collusion, for Goffman (2017, p. 339) there result consequences at two levels: on the one hand, for an excolluded person reality is kept unstable. This changes as soon as the manipulation of the situation is revealed. On the other hand, the relation of the colluded person to the colluding actor is put into question if the former discovers the deceit. Yet, collusion guarantees the maintaining of the social order. Making forget may be understood as a variant of collusion which, however, would have to be pursued further in view of this terminology. 
To continue interpersonal relations, it may well be functional if one or both interaction partners dedicate part of their mutual actions to the denial of indications of the relationship being disturbed - such as mutual trust - or appropriately preparing for encounters. It is an as yet mostly unanswered empirical question in which cultural contexts and interaction situations traces of an irritating past are typically eliminated. This becomes relevant for an oblivion theory as soon as these are indications of a past that will inevitably burden the continuation of a relationship if being discussed. ${ }^{162}$

When it comes to making forget in social groups, a steering impulse or impetus is crucial, guided by certain interests. At first, this is about the purposeful maintaining of group cohesion, which is represented by the holy and is taken care of by priests in traditional communities. Clerics have the task of supervising and safeguarding, purposefully shaping collective oblivion processes, the cohesion of the community.

In secularised social structures, at the meso-aggregation-level, this is the task of leading elites which may be attributed to micro-politics, leadership or management. This does not only hold for populism, among whose repertoire there belongs purposeful distraction from topical fields or debates which are not in line with its interests when the populists' interests are supposed to be successfully presented. Instead, the focus is on any memory or remembrance policy allowing for or intending comprehensive, collective oblivion. Whereas Halbwachs seems rather understand the latter, which must be considered the construed nature of collective memory, as being evolutionary, Aleida Assmann understands it according to Nietzsche's statement according to which it is a "'memory of will' and of calculated selection". Thus, in contrast to individual memory, not compatibility but mostly a "polemic counter-construction to other existing collective memories" is in the fore (A. Assmann, 2002, p. 186). This figure is similar to the idea of construing identity by creating a difference, in the context of which commonalities and similarities are ignored. For Assmann, the manipulability of memory is one reason, among others, why collective memory should be distinguished from cultural memory: due to the artefacts stored by cultural memory, she says, it is less open to the grasp of the powerful who decide what is supposed to be remembered and what is supposed to be forgotten. However, it may be that she overlooks that any cultural artefact is itself a product of selection by the powerful, that only against the

162 Any indications of past love, as well as some traces of the previous life are carefully erased if a new love is pending - Oscar Wilde's novel The Picture of Dorian Gray is a thus-related, nice fiction when, instead of immoral young Dorian, his portrait, hidden in the attic, becomes older (see A. Assmann 2006). 
background of predominant discourses can it be provided with meaning and does not have any meaning a priori.

Also, concerning organisational micro-politics, oblivion management is common practice, for example, enforcing particular or strategic interests and orientations. For example, any presentation - such as a business report or statement of financial position - consists of selected information. In the case of over-complex contexts, only selected facts of the organisation's past activities are accentuated and exposed - others are ignored and "brushed under the carpet". Also there, dealing strategically with oblivion does not only serve for hiding violations of norms but most of all - as finding expression by Goffman's collusion concept (Goffman, 2017, p. 339) - for maintaining the social order as well as the power relations which are currently realised by it.

Furthermore, the realm of organisational learning may be associated with authoritarian oblivion if certain information is no longer available. In the field of organisational narrations, such a kind of oblivion may consist of the disappearance of once established stimuli for memories of old times - such as rights connected to the memory of founding days and claims by the members of the organisation. ${ }^{163}$ In particular, the transfer of personnel may be based on purposefully making forget as a tool of organisational steering. Thus, it is not exclusively about entrusting staff members who do not meet the expectations with different tasks but also about making their assumed wrong decisions fall into oblivion so that the concerned operating unit is ready for a fresh start. Sometimes oblivion is even given as the reason when, after a leading position has become vacant, this position is supposed to not be occupied for some time - although actually, this is due to the management's attempts to save money. ${ }^{164}$

At the level of state or regime, institutionalised making forget may be due to the desire to safeguard the nation's future existence and the power structures or the "system". Ernest Renan's statement that oblivion is almost constitutive for forming a nation may be completed here by a practical aspect of rule (Renan, 2018): oblivion policies are, on the one hand, institutional settings for not discussing specific interests and claims resulting from past events. The function of political steering to coordinate and carry out institutional change always results in forgetting obsolete structural elements, starting or at least

163 Richard Sennett's (1998) descriptions of company restructuring imply comprehensive oblivion processes happening not only in the sense of organisational forgetfulness but are also initiated by the management.

164 The problems for organisational memory connected to such a personnel policy have been pointed out to not only by Christopher Pollitt (2009) but also by Sylwia Ciuk and Monika Kostera (2010). 
tolerating structural oblivion (II). On the other hand, they appear as the flipside of remembrance policies, when memorial days for specific events are not introduced, or historical persons that might be commemorated by naming streets after them are not considered. That no memory is possible without oblivion becomes obvious most of all in this context. Any political decision on memory historicises and this way emphasize certain features in developing collective identity. However, it would be naïve to assume that this is just an addition to the stock of memories. Rather, in the slipstream of remembrance, there happens a much more comprehensive forgetting of everything that would also have been remembered in the given context. Focussing - also of resources on a remembered event is the same as steering and distracts from alternative remembrance options. From the point of view of political decision-making, it may be reasonable to launch little disputed remembrance where problematic memories might irritate the existing order. ${ }^{165}$ Here, the oblivion aspect may be understood in the sense of social overwriting or of screen memory.

Politically launched remembrance is persistent - at least in stable systems. Once established, it is difficult to rededicate or remove a memorial day, a memorial site, a street name, a museum or an archive, but also a law, without good reason, as the thus connected information as well as the ritual or at least routine way of dealing with it has become an element of everyday practice. Thus, in the course of politically motivated remembrance, there also always happens structural amnesia. Then the decision maker's skilfulness decides how far it is possible to forget peacefully or if, as a consequence of his/her actions, ghosts are unleashed.

As it has been demonstrated, the memory-constitutive and always a manipulative variant of making forget can first of all be stated for the context of the social. Crucial in this context - if for once we disregard the possibilities of purposeful medical-therapeutic interventions into memory, which are still at their beginnings - there is the attempt to shape the selectivity of social memories in such a way as to certain memories no longer being accessible. This does not only happen by destroying memory-laden structures or artefacts but also by way of manipulating their meanings.

165 In Spain, the remembrance practice changes with the respectively ruling party or regime one time the victims of the Civil War, another time the victims of the dictatorship are commemorated, sometimes in the course of exhumations covered by the mass media. The way of commemorating and who is commemorated, however, is always accompanied by political considerations. Accordingly, Walter L. Bernecker (2008) states that shortly before the parliamentary elections of 1986 the government abstained from comprehensively commemorating the Civil War, to not alienate right-wing voters. 
Up to here, the above-developed aspects of social oblivion have been dealing first of all with the perspective of social entities. The forgetting of objects as well as the object nature of what is forgotten has hardly been addressed. According to what has been said so far, it must be stated that two sub-processes of the process of oblivion may be distinguished. Oblivion (I), as preparation for an actual loss of knowledge, is subject to social action. The meaningful approach connected to this kind of oblivion happens most of all in the realm of declarative-reflective knowledge and is connected to experience as well as to the shaping of individual and collective memory. For the time being, the actual loss of knowledge, as it has been described by the terms forgetfulness or oblivion (II), is difficult to describe if self-application is concerned, as it works in the realm of pre-reflective practice or the context of incorporatedpractical stocks of knowledge. Thus, oblivion-oriented action always consists of connecting both kinds of oblivion. In the following, we will consider two problems that do not easily fit in with the unfolded classification. On the one hand, it is still about the issue of what has been forgotten and thus about the object perspective. On the other hand, we must still address the effectiveness of oblivion in the context of social structures of meaning.

\subsubsection{Oblivion}

One main problem of the sociological way of dealing with the phenomenon of oblivion is that the forgotten represents something that is no longer existent. Elsewhere it has already been pointed out that oblivion may be considered a special variant of not-knowing. Oblivion (II), defined as the process of overwriting and newly connecting schemes and types, cannot be reflected on from the point of view of an individual or collective consciousness and is at best an observer category. This is addressed if a way of behaviour is supposed to be understood, neither according to situation-appropriate expectations towards the past nor open to being interpreted as purposeful disregard. From the point of view of the forgetting consciousness, one's forgetfulness and the forgotten object can be guessed from traces that are interpreted as indications of currently inaccessible or inexistent knowledge.

The concept of trace has a number of oblivion-theoretically relevant meanings - beyond its use in the deconstructivist context. The trace is not just a disconcerting object which at first evades any meaningful classification; being an indication, it refers to different connections which, however, are at first only vague. Here it is worthwhile to look at the attributions of the trace concept as collected by Sybille Krämer: the trace, being the hollow pattern of an imprint, always indicates absence. Connected to it is an orientation achievement, as the reading of traces does not only consist of action but also 
anticipates future actions. Also remarkable is the aspect of disturbance, as the trace is only perceived if an existing order is irritated. Finally, the trace draws attention to an epochal break by indicating something that is already gone at the moment of being discovered. ${ }^{166}$ At the same time, however, the connection points are still there and may sometimes reactivate older associations.

The trace as an indication of oblivion and that what has been forgotten becomes sociologically relevant most of all if it becomes a subject of communication and makes both the ways of and reasons for oblivion and the forgotten object a topic. In this context, two questions must be taken into consideration. The first one is about why traces may appear which are not adequately recognized but interpreted differently - then it might be that one is "on the wrong track". The second problem results from the first one if traces are noticed that demand remembering without any addressee of this remembering. Such traces refer to aspects of the collective consciousness and are perceived as enigmatic and terrifying as long as they have been dissolved by way of memory. It is obvious to call this figure of a structure-induced revision of oblivion a spectre. In the following, we will have a closer look at the two problems of forgetting about oblivion (1) and of the spectre as a phenomenon of object-centred forgottenness (2).

If one finds out that it has been forgotten that something has been forgotten, at first sight, this seems rather a pun instead of a real problem. In sociological literature, however, we find two hints at forgetting about oblivion. Firstly, Alois Hahn states that the habitus concept, according to Bourdieu, describes such a kind of oblivion. In the case of incorporated memory, not only the realisation of certain practices, that is, learning and rehearsing them, but even the existence of skill is forgotten - at a given time, they are simply practised "automatically" (Hahn, 2007). If, however, one distinguishes between declarative-reflective and incorporated-practical memory, the problem seems to dissolve. Accordingly, the "automatic" or routine selections of the incorporated-practical dimension are characterised by not being remembered in a declarative-reflective way. It is indeed part of the "essence" of routine that its origin and the detailed causality and functions of the way it runs are forgotten. These habits and practices stay declarative-reflectively communicable and thus meaningful because of the narrative form, e. g., of tradition, which provides a legitimating substitute

166 Apart from the already mentioned aspects, Sybille Krämer (2007) discusses the materiality, the lack of motivation, the observer and action dependency, the faculty of being open to interpretation, the one-dimensionality or irreversibility, as well as the mediality of the trace. All these attributes seem to be fruitful for an oblivion-theoretical exploration. Here, however, we must be satisfied with pointing out to the possible potentials of the concept. 
for the routine's forgetfulness of meaning. In other words: the diagnosis of having forgotten about oblivion given the existence of incorporated knowledge results from astonishment about not declaratively remembering this.

A similar concept is double-loop-forgetting, according to Sylwia Ciuk and Monika Kostera, in the case of which, however, not incorporated knowledge is the topic but successful intentional or created oblivion with organisations. If intentional oblivion is successful, nobody knows why oblivion has happened (Ciuk \& Kostera, 2010, p. 5). Precisely double-loop-forgetting provides a starting point for understanding the irritation resulting from the appearance of traces whose origin can at first not at all be explained. Accordingly, it is a popular motif of films and novels that a protagonist who, due to retrograde amnesia, has "lost" his/her (declarative-reflective) "memory" starts decoding his/her origin with the help of incorporated skills that have not been lost. ${ }^{167}$ However, even groups may encounter things connected to a past which is their own but whose existence cannot be sufficiently explained, as, for quite some time, the selections of the social memory have schematized of classified other association patterns. Such enigmatic encounters with the past of one's group have neither been described sociologically nor in any other way, which is why in the following we are going to offer an interpretation labelled, strangely enough, a sociology of the spectre. It might, however, be a crucial element of a sociological approach to social oblivion.

Making use of a concept coming from the "enchanted garden" of preEnlightenment times, which cannot even be declared a common metaphor, is risky indeed. However, it seems necessary to lay out the figure of the spectre as a sociological fact if one understands it as a genuinely social authority connecting oblivion and remembrance. This is meant to say that not at all it is about something supernatural, metaphysical or romantic, as after a sociological clarification of the term, many ghost stories can be explained in terms of the sociology of memory. ${ }^{168}$

The post-structural theory provides several considerations which may be connected to this. For example, according to Jacques Derrida, hauntology starts from the famous first sentence of the Manifesto of the Communist Party by Karl Marx and Friedrich Engels: "A spectre is haunting Europe the spectre of communism" (Marx \& Engels, 2014, p. 74). Derrida asks about

167 Sequences of this kind are found e. g. in the Bourne films by Doug Liman. A systematizing analysis of amnesia motifs in the film genre - among others in view of behaviour routines which are at first unexplainable for the protagonist - is presented by Katja Kirste (2001).

168 A somewhat more detailed depiction of the social spectre is to be found in Oliver Dimbath und Anja Kinzler (2013). 
possible motivations for using this image and identifies parallels to the spectre in Shakespeare's Hamlet. Like the father's ghost appears to the Prince of Denmark, in Marx, the spectre aims at the future - it threatens the existing order and gives cause to change.

If there is something like spectrality, there are reasons to doubt this reassuring order of presents and, especially, the border between the present, the actual a present reality of the present, and everything that can be opposed to it: absence, not-presence, not-effectivity, inactuality, virtuality, or even the simulacrum in general, and so forth. There is first of all the doubtful contemporaneity of the present to itself. Before knowing whether one can differentiate between the specter of the past and the specter of the future, of the past present and the future present, one must perhaps ask oneself whether the spectrality effect does not consist in undoing this opposition, or even this dialectic, between actual, effective presence and its other. One must perhaps ask oneself whether this opposition, be a dialectical opposition, has not always been a closed field and a common axiomatic for the antagonism between Marxism and the cohort or the alliance of its adversaries. (Derrida, 1994, p. 40)

Without further pursuing this dialectic interpretation of the haunting here, it must be stated that, by way of an unexpected reference to the past, the spectre irritates each respective current reality and addresses an alternative future. Thus, any "appearance" or "trace" whose origin can be clearly attributed to the past of a social - individual or collective - consciousness shall be called a spectre. However, it is typical for such a situation that no interpretation routine is at hand for each individual case of the spectre. ${ }^{169}$ Furthermore, it is transitory; as an appearance, it lacks the aspect of materiality. Whereas the trace is always something that has been left behind, an indication in need of interpretation when having insight into a disturbed order, usually the spectre disappears without a trace, to then suddenly reappear. Thus, we must start from an incident that can be observed but shows the trace attributes of the indication and need for interpretation in the context of irritation. Who sees a spectre is thus "automatically" concerned, as it only appears in the context of the past of a social order of relevance. Such a person may be aware of the disturbance, and then he/she must get along with the repeatedly appearing phenomenon, or he/she experiences the process as calling on him/her to pursue this previously unknown disturbance.

169 The reader of traces need not necessarily be an expert of the assessment of indications, as he/she is characterised in Carlo Ginzburg (1989), as the type of the scientist per se, but rather somebody seeking orientation in the sense of Werner Stegmaier's (2007) trace analysis. 
The spectre demands memory without revealing the necessary associations. If the trace is not successfully pursued, and as long as this is the case, and as long as no solution is found that considers the collective consciousness order, it will go on haunting. The isolation or acceptance and inclusion of this spook into everyday life may have been possible in older kinds of society; ${ }^{170}$ in modern society, it is unacceptable. As a knowledge-sociological motif, the spectre is a structural inhibition for oblivion rising from the "corpus" of the social; it might be imaginable that, e. g. an aspect of mechanical solidarity of the collective consciousness has been violated. According to Durkheim, restrictive law demands appropriate punishment, which has not been possible due to certain circumstances. Now, allegedly from the "structure", there arises the demand to retroactively avenge the injustice which has wrongfully been forgotten (Durkheim, 1984) or to create the preconditions for forgiveness and active oblivion in the sense of Ricoeur (2004a).

In this sense - to refer to a famous example from the belle lettres - we may interpret Oscar Wilde's ghost farce The Canterville Ghost in terms of the sociology of memory (Wilde, 2016): Sir Simon de Canterville haunts the castle, not because he has killed his wife, as the legend has it, but because in revenge her brothers bricked him in at a hidden place in the castle. This violation of the collective consciousness of British society, in which an offender was not regularly tried, and the sinful deceased was not regularly buried, results in a spook. However, the story becomes grotesque when the spook is de-contextualised. Oscar Wilde has the family of a US-American diplomat - as a symbol of enlightened-modern American pragmatism - buy the haunted castle along with the spectre. This change of frame and reference group produces the result that no longer Sir Simon confronts the descendants of those who were responsible for his existence as a spectre with the past but that he is confronted with his terrifying behaviour being consequently misinterpreted. The spectre faces the dilemma of no longer having any addressee for his remembrance activities and must - personified as an actor or no longer appreciated and feared as a terrifying spook - be aware of going around as a meaningless bogey in

170 Here, an alternative interpretation of the archaic belief in ghosts becomes apparent, in the context of which spectres are no longer understood in the context of totemism and aninmism but as interpretation systems lastingly unsolved social problems. It is about connecting remembrance and oblivion: in certain situations the collective memory enforces problem-specific remembrance in the sense of interpreting a situation as terrifying, tabooed, dangerous and unsolved. In this context, the terrifying nature of the interpretation causes forgetting about the social origin of an, after all, social - of course solvable - problem. However, the function of this institutional arrangement is still to relieve current action when it comes to "tough nuts". 
all eternity, as nobody seems to be able to understand the, at best, enigmatic remembrance symbolism und to thus solve the riddle and deliver him from his existence as a spectre. Then there comes rescue by the person of the diplomat's daughter who, being British by birth, can act as an interpreter and decipher the cause of the spook. Finally, with the help of a religious-magical ceremony, it becomes possible to deliver the Lord of the castle by creating the preconditions for a regular burial. The social order, which was violated in the past, has been restored.

A spectre is a highly concise while at the same time immaterial trace. It irritates only those whose social past is concerned or those who are involved. Only they have the possibility to pursue it. This opportunity is due to the circumstance that the spectre is understood as such only by those consciousnesses as belonging to a group whose foundations of social cohesion have been shaken. In other words: the spectres of one group cannot be seen by the members of other groups respectively, the traces cannot be recognised or deciphered as such. It is the function of the spectre (as a revenant) to remind to past violations which must be avenged (or compensated for) even in memory - that is retroactively - to restore the damaged consistency and coherence of the collective consciousness. What is at stake is group cohesion in the sense of mechanical solidarity and collective consciousness in the sense of Émile Durkheim. The past violation of cohesion is avenged by the spook. There where this function has become obsolete as a result of modernisation processes, the spectre is effectless.

Typically, the archaic-traditional spectre refers to the ban of murder, and if the murder cannot be avenged, it appears because a) the deed was committed "perfectly" (that is, without any indication of the perpetrator(s)) or b) because it has allegedly been legitimated by way of power/rule. ${ }^{171}$ Crucial in this context is pointing out a violation that would have to be avenged by restrictive law, which puts the community's coherence based on mechanical solidarity into question. Atonement need not necessarily be the punishment

171 For another famous ghost story, here we may refer to Catherine Howard haunting Hampton Court Palace. Being one of the wives of King Henry VIII, she was accused of marital infidelity and executed. Still today (para-)psychologists and natural scientists investigate these spook appearances (see e. g. Wiseman et al., 2003). According to a sociological interpretation, it is an as yet unpunished violation of the sense of justice of British collective consciousness which, concerning this case, has not "come to rest" until these days. In such cases, the members of the concerned collective react particularly sensitively to irregularities of their physical environment - they see ghosts. 
of the long-deceased perpetrators, which cannot be realised, but the correction of memory - for example, by the mortal remains being buried at the right place, which way they are posthumously recognized by society. Probably this kind of spectre has never been completely replaced and still haunts - and that is communities kept together by mechanical solidarity.

In social-scientific literature, the here discussed spectre is addressed although not explicitly - in different contexts. Usually, it appears in the context of issues of social memory or remembrance, and thus it can be discussed concerning its attribution to the sociological micro-, meso- or macro-level.

At the micro-level, on the one hand, it corresponds to Freud's concept of suppressing an injury of the soul in the past. The neurosis appears as the ghost of the traumatised soul, which can be "delivered" through systematic association on the psychoanalyst's couch. On the other hand, such difficult to understand traces are found in close social relationships, with ways of behaviour making an interaction partner suspect that the other hides something from him/her. In this context, it must be assumed that the hiding partner - mostly unaware - leaves traces that indicate that something is wrong - such as blushing or sweating. Thus the deception, camouflage or suppression - as far as there is one - is imperfect, perhaps not even intended, but it appears as an aspect arousing suspicion, which finds expression by phrases such as "there is something between us".

The collective consciousness of the social group (meso-level) seems to be the reference point of the spectre's function, in the context of which some shifts of emphasis are necessary when it comes to analyses of modern societies. The modern spectre appears in functionally differentiated contexts, in the guise of a "bad conscience", and there it does refer to violations of the institutional order or the coherence principles of modern societies in general. Like the archaic spectre, here it is about "damage" that has not been compensated for by restrictive law and cannot be effectively repaired by restitutive law. In other words: there is an attack on the principle of organic solidarity and not a violation of individual, functional-organic and balanced orders.

At the social macro-level, we find large collectives and social regulation systems in the sense of institutions, norms and values. There the identification of spectres becomes difficult. The structural aspect of the macro-level does not know any disorder and does not have any sense of guilt or right and wrong. Thus, there is no addressee for oblivion being prevented - except we assume a spectre aiming at the national consciousness. Then, the appearance of Catherine Howard would be perceivable, particularly for English people. 
Metaphorically, the terror by the Rote Armee Fraktion is understood as the spectre of Germany's post-war society. ${ }^{172}$

The spectre may also be understood as an effect resulting from different tempi of oblivion at different aggregation levels. For example, politically initiated making forget (oblivion (I)) may consist of being silent - normatively legitimated - about certain violations of the rights of social minorities from which there result in expectations of compensation. By problematic topics officially not being allowed to be mentioned, the compensation claim is passed on or habituated at the micro-level. Then to the later generations, a thus resulting, possibly passed on, "hereditary enmity" appears as a spectre, until either they have been divulged in the "secrets" of this enmity or they are capable of lifting the "curse" from the past. ${ }^{173}$

After this way, the previously unfolded oblivion systematics have been completed by questions resulting from the problem of object-related oblivion, the second section of this study shall once again be summarised in somewhat more detail. Only then will it become possible to apply the oblivion-theoretical set of concepts to the institution of modern sciences and find out about the oblivionism diagnosed there.

\subsubsection{Search Heuristics for Ways of Social Oblivion}

So far, remembrance and oblivion, some crucial connection points for a sociological interpretation of social oblivion, have been assessed regarding the connection between memory. These considerations can be made more plausible by way of two diagrams. The complicated connection of knowledge, experience, remembrance and oblivion in the context of ontological constitution and identity construction can be illustrated by Figure 2 . It depicts one stage out of a sequence of steps of the genesis of knowledge whose basic motif is an interaction of existing knowledge structure (knowledge (I), stock of knowledge or frame) and a perceived experience, an event or a situation. The thus connected interpretation process results - in analogy to a dialectic figure - in a synthesis called new knowledge (II).

172 The film "Die innere Sicherheit" [Inner Security] by Christian Petzold (200o) tells the story of former RAF terrorists who must return to Germany after a having been on the run for a long time. The working title was "Spectres", and indeed the protagonists of this road movie act as a spook from the past of post-war Germany.

173 The historical or legitimatory preconditions for such situations are described e. g. by Helmut König in his reconstruction of the Germans' way of dealing with the Treaty of Versailles. "Indeed, actual combat comes to an end at some time, but the war of the minds cannot be reversed just by a simple declaration of will or intent in the context of a treaty" (König, 2011, p. 51). 


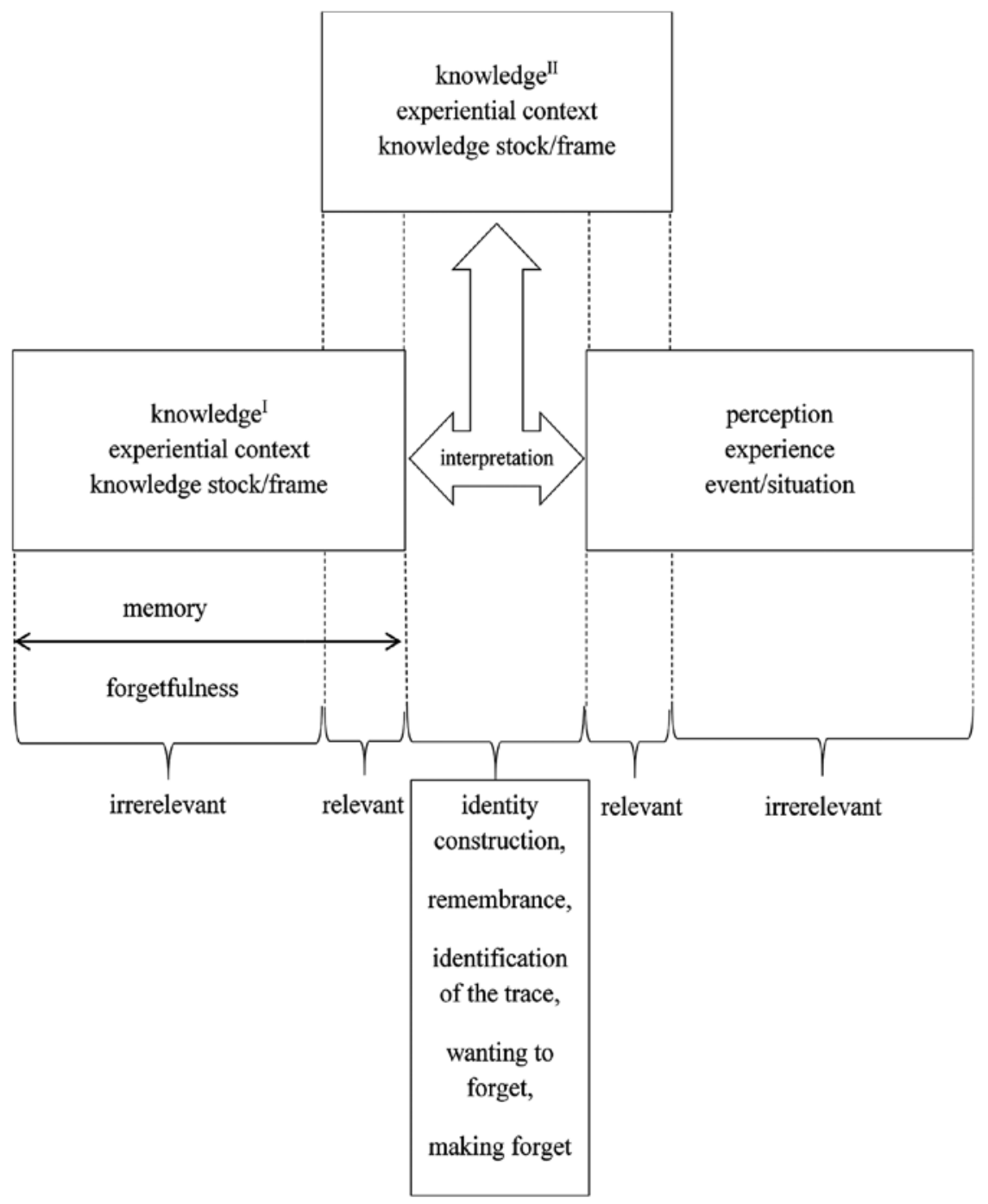

Figure 2 Oblivion in the process of the genesis of knowledge 
In the course of adjusting the situation and the available knowledge to each other, certain experiences and certain perception information prove to be relevant and others not. Figure 2 demonstrates that each new state of the stock of knowledge consists of modifying existing schemes and results from the synthesis of interpretative knowledge (knowledge (I)) and perceptions that, based on existing schemes and frames, are considered relevant. Unaddressed knowledge is ignored and falls into oblivion (oblivion(II)).

The performance of memory consists of selecting relevant schemes and connectable nexuses, which is why the "extent" of what is adopted and what is left to oblivion is different from case to case. In the same way, that kind of perceived information is not considered irrelevant for the new knowledge state. ${ }^{174}$ However, they need not be forgotten, as they are no element of the context of experience or stock of knowledge. In the realm of interpretation, the necessity of remembering results from communicating between existing knowledge and perception. This results from discovering traces of oblivion as a conscious construction of identity. There is also the insight of no longer wanting to address certain existing, declarative-reflective knowledge and wanting to forget it, and likewise the intention to make such knowledge inaccessible to others.

Figure 3 illustrates the intersubjective connection of the various kinds of oblivion. At first, the declarative-reflective and the incorporated-practical dimensions are distinguished from each other - two fields that may also be called the conscious and the pre-conscious level. By the example of the simplest case, that is, the dyadic interaction between ego and alter, the complex and double-contingent "play" of the three kinds of oblivion is demonstrated. The direction of the arrows gives the effect of oblivion processes. Forgetfulness as a pre-conscious loss of knowledge reduces the

174 This is basically a figure similar to the one to the left, as according to Schütz's relevance theory also perception is guided by schemes and thus in a memory-specific, selective way. However, here we cannot speak of oblivion or forgetfulness, as one does not at all become aware of the not selected perception information. Giddens (1984) describes this situation by reaching back to psychological analyses in the context of which the scheme-oriented selectivity of perception is made plausible; in Luhmann (2012, p. 351) the problem is indeed mentioned but not clearly solved, as there the system memory selects the perception information, to then forget about everything not being congruent with the "system as reality". It is thus assumed that the "consciousness" or the perception apparatus at first adopts everything, to select subsequently. 
consciousness's capability of reflection. In the case of ego, starting from the consciousness, wanting to forget aims at unconscious knowledge. From the perspective of the individual consciousness, wanting to forget must be equated with making forget - in the sense of self-objectification. Now, by way of interacting with alter, the ego may try to influence the alter's conscious as well as pre-conscious knowledge.

The following will be about integrating these manifold aspects as far as possible and developing a scheme of interpretation from this. For this purpose, some guideline distinctions will be made (1) by way of which findings on the social functions of oblivion can be further systematised (2). Then, to conclude, these perspectives will be located in view of the distinction according to social theory and theory of society, as it has already been unfolded at the beginning of this study (3).

As has become apparent in the course of our considerations so far, the structural-reconstructive work at the phenomenon of social oblivion requires quite a number of differentiations. The benefit of such a differentiation is that social aspects of oblivion can be determined in more detail. By introducing further differentiations derived from sociological order semantics, a scheme has been developed with the help of which several oblivion motifs and oblivion equivalents to be found in the sociological debate can be systematically organised.

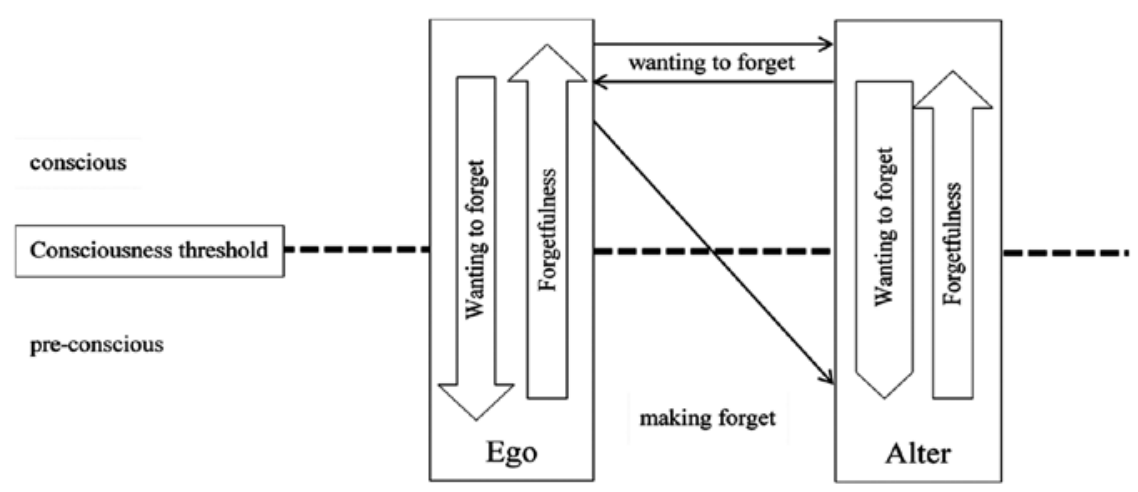

Figure 3 Intentional und unintentional oblivion

From an overall view, the manifold kinds and possibilities of social oblivion can be depicted as follows: 


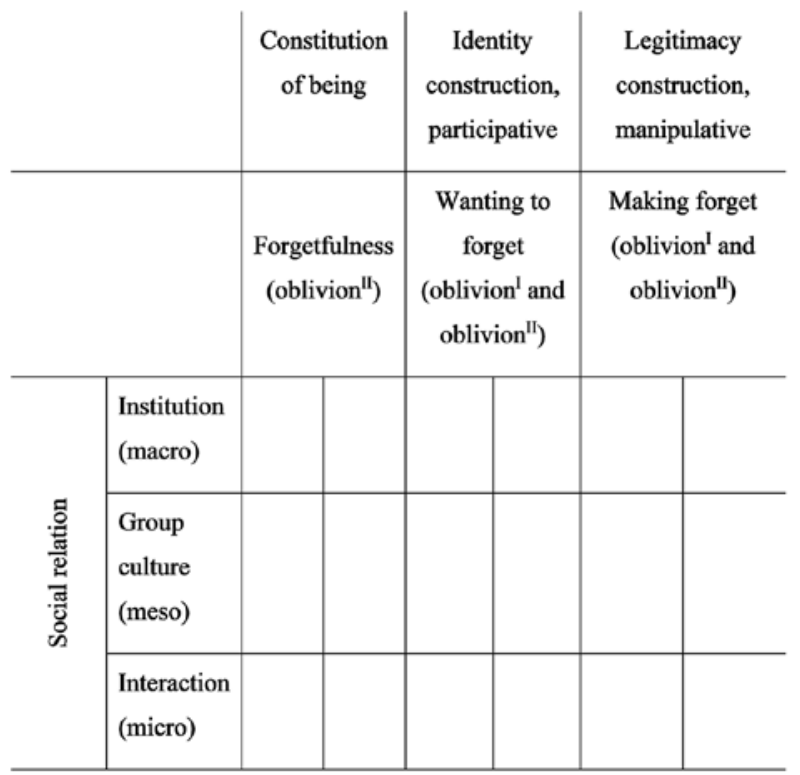

Figure 4 Search and interpretation heuristics for kinds of social oblivion

The levels of the social, which above are distinguished by micro, meso, and macro, which are understood to be orders of social relations becoming manifest by interactions, group cultures and institutions, also correspond to the concept of social reference frames. By way of the latter, it has been possible to categorise the different levels of phenomena of social oblivion. Constitutive for distinguishing the kinds of social oblivion of forgetfulness, of wanting to forget and of making forget is delimiting the oblivion process - as oblivion in the stricter sense of oblivion (II) - from the preconditions for this oblivion, which have been included as oblivion in the wider sense of oblivion (I). Whereas the concept of forgetfulness depicts the process nature of oblivion, ${ }^{175}$ both wanting to forget and making forget appear as combinations of the activity-mediated and intentional production of making disappear, no matter in which way, both material and cognitive objects as well as the expectation, which is connected to this intention, that forgetfulness will start. This kind of forgetfulness consists of preventing remembrance.

175 It is thus not about the tendency, common in everyday language, of easily forgetting something but about the fact that something can be forgotten and this ability to forget is a universal process. 
If forgetfulness is supposed to be used strategically, the change of schemes, scripts, frames, or types embedded in contexts of meaning or experience or in the stock of knowledge comes into view. However, such a change does not consist of any substantial change of shape but the restructuring of the embeddingor connection-structure so that associations with an object which is supposed to be forgotten or with a context of meaning are separated as much as possible from each respective relevance attribution. This requires the removal of remembrance stimuli. ${ }^{176}$ It may also be imagined that the knowledge of a certain event is completely ignored. Even in this case, the knowledge is not lost, and it may be - although in a different shape - restored in the course of a memory-constructive search for traces, or it may be provided with a new meaning.

The difference between wanting to forget and making forget is that, in the case of wanting, work on the social construction of reality, which is about social action in the Weberian sense, happens more or less participative and in accordance with the participating consciousness bearer. In the other case, it is manipulative strategies for which actors exploit the principles of forgetfulness and try to influence an existing knowledge structure. A mixed kind of wanting to forget and making forget develops in the context of authoritarian regimes or asymmetrical relations, which may bring the will of the collective in line with the interests of rulers.

Metaphorically spoken, when it comes to consciousness, the oblivion process which has been characterised as forgetfulness may be compared to an iceberg, with only its peak being declarative-reflectively accessible to conscious perception. The by far larger part constitutes knowledge in the incorporatedpractical mode, which leads to the conclusion that the process of oblivion can hardly be experienced, although it considerably co-constitutes the being of each respective entity. Thus, in this context, we may speak of forgetfulness as a constitution of being, in the context of which most of all the process and less the state and structure of a being is depicted. The other two kinds of oblivion are construction methods that happen mostly intentionally and can be grasped or experienced consciously or discursively. In the case of wanting to forget, after all, it is about construction processes that - as self-attribution or attribution by others - aim at identities. In the case of making forget, on the

${ }_{17}$ Connecting to more recent debates, this may also be called experiential knowledge (see e. g. Böhle et al., 2001). However, as the concept of experience according to the knowledgesociological understanding is used differently, and as the equivalent given as "traditional action" among the reasons for action in Weber (1979) is not suitable, Schütz's concept of "habitual knowledge" appears to be more appropriate here (Schütz \& Luckmann, 1973). 
other hand, rather the construction or preservation of legitimacy or legal rule is at the fore.

Given the broad range of interpretations of the loss of knowledge as social oblivion, now given oblivion's theoretical benefit, we may further ask about the social function of oblivion. The starting point is three kinds of oblivion, from whose interpretation as a constitution of being, of identity- and legitimacyconstruction it is possible to derive social or societal functions. Thus, the constitution of being, communicated by forgetfulness, guarantees orientation for a social entity that is rather incorporated-practical instead of declarativereflective and meaningful. In exceptional cases, this orientation may serve as the foundation for relevant experience based on past experiences, for only part of this interpretation of the presence is based on declarative-reflective remembering. The overwhelming share of knowledge consists of incorporated routines and practices taken as a matter of course and are not explicitly discussed. The memory selects what is relevant by way of a process of social adjustment. In this context, the memory process consists of connecting or associating what is perceived in a given situation with existing association patterns in schemes, scripts or types. As this happens by way of a similarity comparison, it stays at first an open question if this connection happens by way of a similarity connection (redundancy) or the exclusion of dissimilarities (variety). At the same time, at the moment of renewed, reminding connection and adjustment, a time index is inscribed into the modified structure, ${ }^{177}$ which works in favour of a selection according to "novelty" or "actuality". The flipside of the results of this double memory process is described as oblivion in the sense of forgetfulness. Fallen into oblivion has everything which is not currently used for finding orientation in an emergent situation. This is both due to lacking similarity estimation and to the chronological distance or lacking relevance.

However, this way has fallen into oblivion must not be understood to have disappeared but to have not been used. It is an element of the stock of knowledge in potentialis; that is, it is basically available for associative connection to define the present. Regarding the ontological status of oblivion, it may be stated that it is incorporated "knowledge" whose activation can only in cases of exception be controlled by consciousness. Then forgetfulness - as a memory process - develops by way of ignoring schematised or classified knowledge

177 That this happens only in exceptional cases, as Luhmann (2012, p. 350) believes, is only partly correct. Only in rare cases, if the date is obviously relevant, a scheme will be parallel connected to a corresponding time scheme. A scheme-immanent indexing of time, however, creates the basis for the inherent history of a scheme in the sense of being "unprecedented". 
structures. However, as an associative context resulting in unperformed current connections may be connected to many other associative contexts, again and again, it is possible to experience references to rarely used patterns - the delay and the difficulty of newly creating or reactivating such connections has its equivalent in experiencing the attempt to remember forgotten things.

The flipside of positive memory selectivity as defining relevance has the function of excluding other possibilities of the presence-constitutive performance of orientation. As only connected association patterns are provided with an updated time index, any case of not connecting or not activating reduces the probability of any new recall. As we might have it illustratively, the social entity flows in the bed of its continuity which, as a result of the power of flowing, changes slowly but stubbornly - progress under the pressure of what has been. As here - in contrast to the individual consciousness - the selectivity of the memory must be theoretically modelled, an analysis of the social function of forgetfulness provides insights into the construction of each respective being's boundaries to its each respective environmental context. That naming and investigating this function is constantly confronted with the non-declarative or incorporated-practical makes it scientific exploring a core problem of knowledge-sociological thought at the level of reality constructions second order. ${ }^{178}$

These both complex and abstract considerations on the social function of forgetfulness as a quasi-natural oblivion phenomenon related to non-natural concepts of social-scientific analysis are the starting point for defining the functions of the two other kinds of social oblivion. Both wanting to forget and making forget are based on - this has already been pointed out to - knowledge of the process of oblivion. In any case, the conscious dealing with ideas of the effect of oblivion consists of the assumption - somewhat counterintuitive at first sight - that oblivion can create order. Against this background, for the actors, oblivionist oblivion appears to be a tool with the help of which memories and thus the selectivity of orientation-providing knowledge can be organised.

178 From Schütz's (1967) social-phenomenological point of view, the determining of the social function of forgetfulness cannot be about reconstructing the 'effect' of oblivion processes but must be about investigating the meaningfulness of such a kind of oblivion in the context of social-scientific observation categories. In other words: the sociologist is incapable of immediately researching social oblivion, but he/she depends on his/her own models or concepts of the social - indeed analysis units which are not 'naturally' given and are already 'artificially' defined categorisations: groups, collectives, discourses, and so on. 
In this context, it seems as if two patterns of interpretation determine such a way of taking oblivion into service. Firstly, any scientific understanding rests on the idea that the future could be controlled by artificially creating forgetfulness. On the other hand, secondly, any technocratic understanding comes from the assumption of being able, by way of manipulating the framework conditions for possible remembrance, to control "natural" forgetfulness and to this way also control the present future and, as a consequence, also future presences (Luhmann, 1976). Whereas in view of the social the first variant still rests mainly on some naïve, blind faith in science or on dystopic science-fiction and, for the time being, is most of all discussed in science-ethical debates on science assessment, ${ }^{179}$ the second variant has always been an element of (microor everyday) political practice. Both kinds are sociologically relevant if they are distinguished according to participative (wanting to forget) and manipulative (making forget) tendencies. The social function of both wanting to forget and making forget consists of shaping presence-related definitions of situations which also aim at future action. In contrast to "natural" forgetfulness, however, here it is about intentionally shaping the social order - although the results of appropriate actions particularly generate side effects and are unstable as well as mostly uncertain.

Given the different implications for the genesis of social constructions of knowledge and thus also for the social order, one may ask if the issue of social oblivion might also provide independent starting points for the development of a sociological theory or if we should leave it at a "phenomenology" or "sociological analytics". 180 The following shall thus be about assessing at least the possibilities of a theory of social oblivion.

As has been worked out so far, social memories are said to have contextspecific and universal qualities. Among the universal qualities, there belongs firstly, in analogy to the insights concerning the functioning of the human brain, the capability of structural genesis. The development of order results from the "experiences" of social entities and allows them to adjust to their environment by developing a system of successful reactions, among which, since the existence of language, there also counts symbolic schematisation or classification. Furthermore, the circumstance of the situation-sensitive selection of

179 The feasibility of pharmacologically induced oblivion is discussed in the contributions to Volume 9, issue 7, of the American Journal of Bioethics (2007).

180 Peter Wehling asks a similar question, by at first not at all intending to develop a theory of social oblivion and then attempting to "react, in a theoretically appropriate way, to the heterogeneity and multi-dimensionality of oblivion as a social phenomenon" (Wehling, 2011a, p. 15). For the start, he leaves it at looking for elements or tools of sociological oblivion analytics. 
possible associations in view of an existing structure or pattern of information processing must be considered. Thirdly, the construction of timeliness may be understood to be an independent scheme that feeds on a time index inscribed into the schematisation processes.

When assuming that the performance of social memories develops further in a stubborn way, according to the assumptions of functional differentiation, the reconstruction of differentiable ways of selecting produces the result of field-specific social memories. This way, it becomes possible to connect the principle of memory to the concepts of the subject, dyad, group, collective or nation and also to the thus connected ideas of order in the sense of identities, discourses, systems or institutions.

Furthermore, if we assume that these social entities constitute themselves more or less stubbornly, the category of memory becomes a crucial theoretical element of the social-scientific description and analysis of this constitution process. In other words: only the concept of social memory provides us with an appropriate conceptual tool set for the analysis of the path-dependency of social change beyond any framing by - always meaning-communicated - historiography and historical science. In a twofold way, social practice theories, which have been dealing with such blind spots for quite some time, are not helpful. Firstly, the concept of practice is ambiguous, as it also always implies practice as the opposite of theory and is used as a counter-concept to a kind of science that is relieved from everyday pressure to act. Secondly, in the wake of a practical turn, many social circumstances cannot exclusively be described as practical, routine-guided, habitual or unintentional. Rather, a concept is needed to understand explicit and implicit knowledge with an admittedly necessary reference to the past as being authoritative for situative orientation. Thus, the crucial aspect is how each analysed social context constitutes itself by reaching back to its "past" concerning the knowledge structure to be diagnosed. Parallel to the entities of the social to which, as an individual or collective consciousness, a memory may be attributed, in view of the ordering principles of the social we speak of memories. However, keeping this apart would result in terminological difficulties when it comes to the already existing terminological practice, which is why in the following, we will speak of the memories of systems and institutions - although obviously, we will look at individual selection mechanisms.

The question about social oblivion shares this basic assumption. It accentuates the memory's selection mechanisms and focuses, on the one hand, on those selection practices as being connected to category-specific ways of constituting. On the other hand, it is interested in the circumstance that any orientation or adjustment performance indeed provides for the possibility of 
other connections resulting from the past of each entity but is, for obvious reasons, not taken up. In other words: any social entity does not only "know" much more than it believes to know and can declarative remembering. Also, it "knows" more than needed for the routine operations of organising its selectivity-communicated orientation.

The difference between what is provided by the (social) experiential context and what is practically used and consciously communicated is what has been forgotten - knowledge that exists but is currently unavailable. The processes and mechanisms constituting that what has been forgotten are a differentiated context of different ways of forgetting. Of particular interest for sociological analysis in this context are those processes as being based on their schemes of oblivion. Thus, the focus is on the hypotheses-guided analysis of practices and strategies of oblivion in social relations, which are meant for creating social order. However, in contrast to the closely related memory processes or to the process of remembering, here rather a kind of "de-memorising" is at the fore, that is, attention turning purposefully away from memory-specifically expectable relevancies. If the interest in such processes is focused on constituting meaning or reasoning, we enter the realm of what may be called oblivionism: intended or created oblivion as socially meaningful action.

Whereas the analysis of the oblivion process, which here has been presented as forgetfulness or oblivion (II) as a specific way of schematisation or association, may already reach back to comprehensive theoretical preliminary work - in particular from the fields of psychology, the neuro-sciences and philosophy ${ }^{181}$ it seems as if the social use of oblivion, in the sense of its action-practical exploitation, has as yet been hardly described and assessed for its thus-connected potential for sociological investigation.

According to the quality standards of Weber's adequate causation, the critical analysis of historical processes of the change of the meaning of terms, discourses, institutions, thought styles or identities is certainly difficult yet realistic in the context of the standards of adequate meaning. The empirical analysis must start from indications for certain memory-relevant topics having been eliminated, being eliminated or being supposed to be eliminated. The social process resulting in or legitimating such actions informs about the schematicclassified idea of social figurations or cultures. Furthermore, this theoretical perspective allows for viewing both at the range and way of functioning of

181 As has been shown, even genuinely sociological approaches are oriened at these theoretical traditions: Schütz is inspired by the philosophies of Bergson and Husserl, Luhman also integrates findings from the neuro-sciences and cybernetics. 
its own forgetfulness and at those associative contexts of meaning serving as the foundation for those cleaning efforts as making oblivion possible. Thus, both oblivion (I) and oblivion (II) as well as their combinations of wanting to forget and making forget, must be considered an explicans, that is, statements by way of which certain social facts can be explained. In focus are both past realities and those being close to the present of the analytical-specific kind, just like time-diagnostic diagnoses of processes for which particular aspects of oblivion could be proven to be sub-processes. In other words: the necessary connection of a structural theory to an action theory of social oblivion may be applied to any level of social relations. 